\title{
Lebanon: Use of Fund Resources-Request for Emergency Post-Conflict Assistance- Staff Report; and Press Release on the Executive Board Discussion
}

In the context of the use of Fund resources-request for emergency post-conflict assistance, the following documents have been released and are included in this package:

- the staff report for the Use of Fund Resources-Request for Emergency Post-Conflict Assistance, prepared by a staff team of the IMF, following discussions that ended on March 20, 2007, the officials of Lebanon on economic developments and policies. Based on information available at the time of these discussions, the staff report was completed on March 30, 2007. The views expressed in the staff report are those of the staff team and do not necessarily reflect the views of the Executive Board of the IMF; and

- a Press Release summarizing the views of the Executive Board as expressed during its April 9, 2007 discussion of the staff report that completed the request.

The documents listed below have been or will be separately released.

Letter of Intent sent to the IMF by the authorities of Lebanon*

Technical Memorandum of Understanding*

*Also be included in Staff Report

The policy of publication of staff reports and other documents allows for the deletion of market-sensitive information.

To assist the IMF in evaluating the publication policy, reader comments are invited and may be sent by e-mail to publicationpolicy@imf.org.

Copies of this report are available to the public from

International Monetary Fund $\bullet$ Publication Services

$70019^{\text {th }}$ Street, N.W. • Washington, D.C. 20431

Telephone: (202) 623-7430 • Telefax: (202) 623-7201

E-mail: publications@imf.org •Internet: http://www.imf.org

Price: $\$ 18.00$ a copy

\section{International Monetary Fund}

Washington, D.C. 



\section{INTERNATIONAL MONETARY FUND}

\section{LEBANON}

\section{Use of Fund Resources—Request for Emergency Post-Conflict Assistance}

Prepared by the Middle East and Central Asia Department

(in consultation with other departments)

Approved by Juan Carlos Di Tata and G. Russell Kincaid

March 30, 2007

- Discussions on the authorities' request for emergency assistance took place in Beirut March 8-20. The staff met with the governor of the central bank, the minister of finance, other senior officials, and World Bank staff. The staff team comprised Messrs. Gardner (head), Schimmelpfennig, Sdralevich, and Ms. Oner (all MCD), Mr. Le Borgne (FAD), and Mr. Tzanninis (PDR); it was assisted by Mrs. Weikert and Ms. Winkler (both MCD). Ms. Choueiri (OED) attended the policy meetings.

- In the attached Letter of Intent (LOI), the Lebanese authorities request a purchase of 25 percent of quota (SDR 50.75 million) under Emergency Post-Conflict Assistance (EPCA). In the view of the staff, the authorities' medium-term reform program presented at the international donors' conference of January 25, 2007, in Paris ("Paris III"), constitutes an adequate basis for an EPCA request, and is presented as an attachment to the LOI as their Memorandum of Economic and Financial Policies.

- $\quad$ Fund support through EPCA is a key part of a concerted international effort to provide financial assistance to Lebanon. A large number of bilateral donors and multilateral agencies are supporting the reconstruction and reform of the Lebanese economy. 


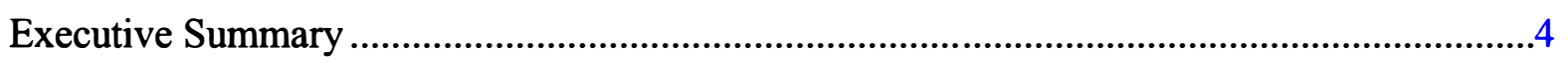

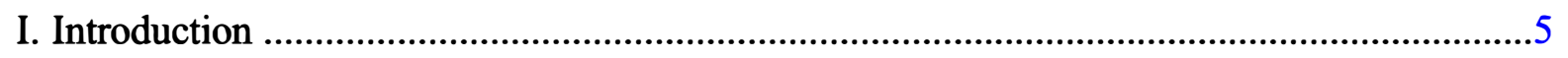

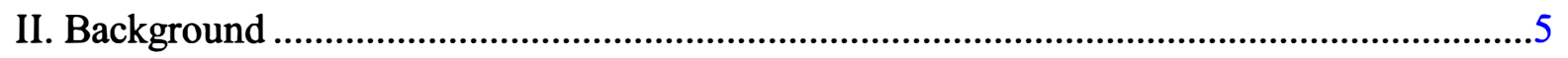

III. Policy Discussions ............................................................................................... 9

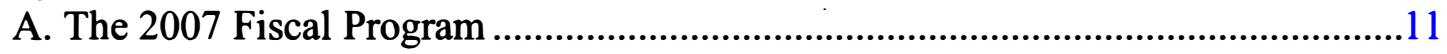

B. Monetary and Exchange Rate Policy ........................................................... 13

C. Structural Reforms ....................................................................................14

D. The Medium-Term Fiscal Program and Debt Sustainability Analysis ...................15

E. The Paris III Package and Commercial Banks' Contribution ...............................16

F. Access, Program Monitoring, and Capacity to Repay the Fund............................ 18

IV. Staff Appraisal

\section{Boxes}

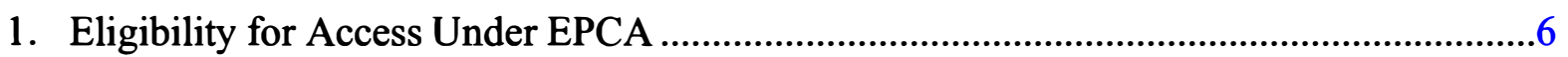

2. The Authorities' Five-Year Reform Program.......................................................10

3. Shock Scenarios for the Debt Sustainability Analysis..................................................18

\section{Figures}

1. Real GDP and Coincident Indicator, 1994-2006

2. Inflation, January 2005-December 2006.

3. Broad Money and Deposit Dollarization .................................................................... 7

4. International Reserves and Liquidity ................................................................

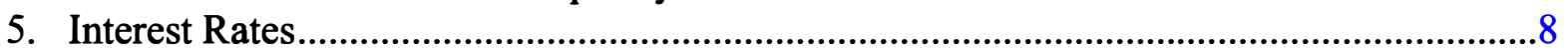

6. Eurobond and Credit Default Swaps (CDS) Spreads .................................................

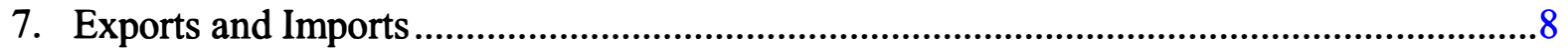

8. Effective Exchange Rates ................................................................................

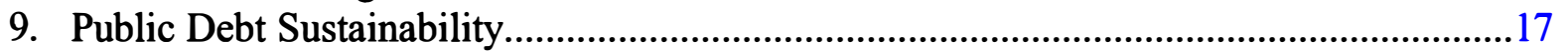

Text Tables

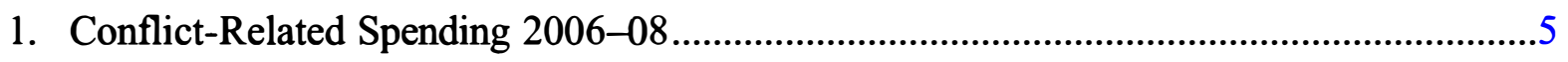

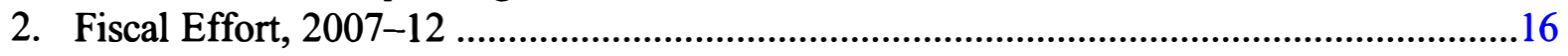

Tables

1. Selected Economic Indicators, 2003-12 .............................................................. $\frac{22}{23}$

2. Central Govemment Primary Balance, 2003-08 (in billions of Lebanese pounds) ........... $\frac{23}{24}$

3. Central Govemment Primary Balance, 2003-08 (in percent of GDP) ..............................24

4. Overall Fiscal Deficit and Financing, 2003-08 ….................................................... $\frac{25}{26}$

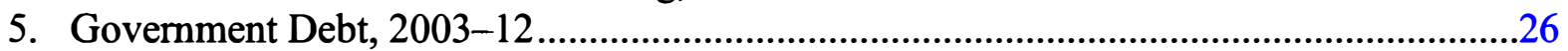

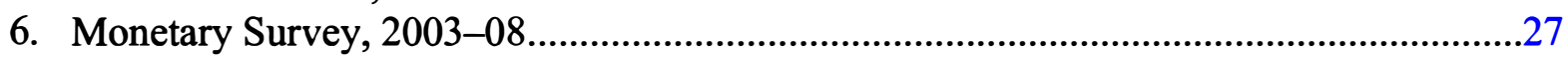

7. Balance Sheet of the Banque du Liban, 2003-08 .....................................................28 


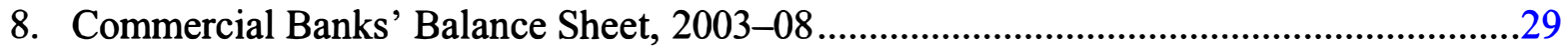

9. Balance of Payments, 2003-12 ……………...........................................................

10. External Financing Requirements and Sources, 2003-08 ……......................................

11. Indicators of Capacity to Repay the Fund, 2003-08 ....................................................

12. Indicators of Financial and External Vulnerability, 2002-06 ......................................33

13. Banking Sector Financial Soundness Indicators, 2002-06...............................................34

14. Public Sector Debt Sustainability Framework, 2003-26 ...................................................

Appendix

Letter of Intent (LOI) .......................................................................................................

Attachments to LOI

I. Quantitative Indicative Targets Under Emergency Post-Conflict Assistance,

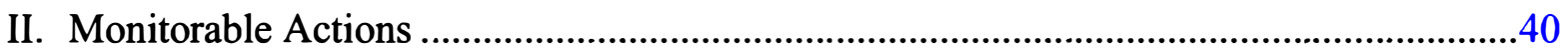

III. Authorities' Medium-Term Reform Program Presented at the Paris III Conference.......... 41

IV. Technical Memorandum of Understanding ………….................................................... 


\section{EXECUTIVE SUMMARY}

The authorities have requested Emergency Post-Conflict Assistance (EPCA) in support of their 2007 economic program, with access of 25 percent of quota (SDR 51 million). EPCA is a key part of a concerted international effort to provide financial assistance to Lebanon.

\section{Background and recent developments}

The five-week conflict with Israel in 2006 and the month-long blockade that followed inflicted a heavy human and economic toll on Lebanon. Donor conferences in Stockholm on August 31, 2006 and Paris on January 25, 2007 (Paris III) generated significant donor support that has mitigated the conflict's impact on public finances. Financial markets weathered the conflict surprisingly well, reflecting the authorities' skillful management of exchange rate pressures, the banking system's strong liquidity position, and deposits by Saudi Arabia and Kuwait with the central bank shortly after the conflict erupted.

\section{Outlook and policy discussions}

- The authorities expect 2007 to be a very difficult year. Political tensions could intensify ahead of the presidential election in the fall and adversely affect economic activity and private capital flows.

- The fiscal program for 2007 aims at containing the primary deficit while accommodating reconstruction and relief spending. Financing of the deficit is subject to uncertainties relating to the availability of donor support and volatile market conditions. The authorities will take steps toward the introduction of a global income tax, social and energy sector reforms, and privatization of the telecom sector.

- The authorities consider the current level of international reserves sufficient in case of renewed financial markets pressures.

\section{Staff appraisal}

- The program for 2007 strikes a careful balance between post-conflict needs and the challenges posed by the large public debt overhang. Implementation of the Paris III reform agenda combined with pledged donor support would significantly lower the debt-to-GDP ratio over the next five years in the absence of adverse macroeconomic shocks. Still, risks in both the short and the medium term are substantial, and every opportunity should be seized to advance the reform and adjustment process. Donor support needs to be closely aligned with the authorities' macroeconomic objectives and policy priorities. 


\section{INTRODUCTION}

1. The authorities' economic program for $\mathbf{2 0 0 7}$ derives from the broader medium-term reform strategy presented at the donor conference on January 25, 2007, in Paris ("Paris III"). The medium-term reform program was developed prior to the conflict with Israel. At the Board meeting on the 2006 Article IV consultation, on May 8, 2006, Executive Directors had expressed their support for an earlier version of the debt reduction strategy, although a number of Directors considered that the size of the imbalances called for a more rapid pace of fiscal adjustment. In the wake of the July-August 2006 conflict and its economic and political fallout, the sequencing of reforms and the timing of fiscal adjustment were changed. The specific objectives of the program for 2007 to be supported by EPCA are to protect financial stability, contain the budget deficit during this transition year, and initiate structural reforms that are critical to the success of the medium-term reform program.

\section{EPCA would provide an appropriate transition to 2008, when fiscal adjustment} is envisaged to commence. Staff's judgment is that Lebanon meets the criteria for Fund support under EPCA (Box 1). The authorities have indicated that they intend to seek Fund support through a Stand-By Arrangement (SBA), following satisfactory implementation of the EPCA program, and after the immediate impact of the conflict has been addressed, and the current political stalemate resolved.

3. The political situation remains difficult, with a risk that legislative activity could be paralyzed in the period leading up to the presidential election, which is expected to be held by the Fall of $\mathbf{2 0 0 7}$. Efforts to end the political crisis have so far not met with success, with the government and the opposition remaining firmly entrenched in their positions, putting parliamentary activity on hold. The security situation has become calmer since the street violence and the bomb attacks of January and February 2007. Despite the political stalemate, the government is determined to move ahead with its reform agenda, and is counting on reassurances provided by the Speaker of Parliament, who is aligned with the opposition, that he would, on an exceptional basis, convene parliament to consider legislation related to the implementation of the Paris III program.

\section{BACKGround}

4. The five-week conflict with Israel in 2006 and the month-long blockade that followed inflicted a heavy human and economic toll on Lebanon. The damage to infrastructure is estimated by the government at $\$ 2$ billion (9 percent of GDP), just over half of which is housing (Text Table 1). Immediately

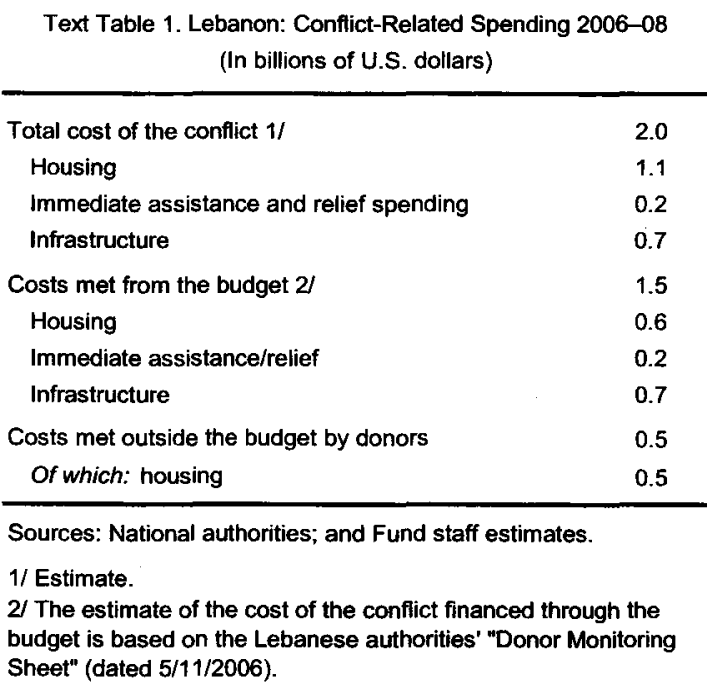


after the conflict, donors committed $\$ 1.7$ billion for relief and recovery. ${ }^{1}$ Most of the pledges are expected to be disbursed by end-2007, but due to implementation capacity constraints, actual conflict-related spending will spill over into 2008 .

\section{Box 1. Lebanon: Eligibility for Access Under EPCA}

Urgent balance of payments need. While the central bank was able to protect its reserves position through the conflict, further balance-of-payments support will be needed in 2007 to maintain confidence in the face of continued political instability, potential market volatility, foreign currency debt service of about $\$ 3$ billion, negative deposit growth since the beginning of the year, and the roll-over of short-term deposits of over $\$ 60$ billion. The projected conflictrelated widening of the current account deficit will increase these vulnerabilities further. The timely deposits for US $\$ 1.5$ billion by Saudi Arabia and Kuwait also indicated the urgency of the balance of payments need.

Disruption of institutional and administrative capacity. The war and its direct aftermath led to a significant weakening of institutional capacity (evidenced by vacancies in key ministries and the impossibility of convening parliament) and stretched the capacity of the administration (due to the added tasks of planning for recovery, reconstruction and donor coordination) to the point where the authorities are not yet able to implement a comprehensive economic program that could be supported by an upper credit tranche Fund arrangement.

Sufficient capacity for policy planning and implementation and demonstrated commitment. Capacity and commitment are adequate, as demonstrated by the authorities' management of financial market tensions and the medium-term economic and reform program, which was finalized after the conflict.

Fund support as part of a concerted international effort. Access under EPCA is small relative to the country's financing needs, but constitutes an integral and catalytic part of the overall financial assistance package of Paris III.

5. The conflict and the ensuing domestic political tensions dashed hopes for a strong recovery in 2006 (Table 1). The latest indicators suggest that real GDP was flat in 2006, compared with a pre-war projected growth of around 6 percent, translating into a loss of national income of about $\$ 1.3$ billion. At the same time, inflation accelerated due to supply shortages triggered by the conflict, reaching 7 percent year-on-year in December 2006.

\footnotetext{
${ }^{1}$ This includes the amounts pledged at the Stockholm donor conference of August 31, 2006, as well as separately by Arab donors.
} 

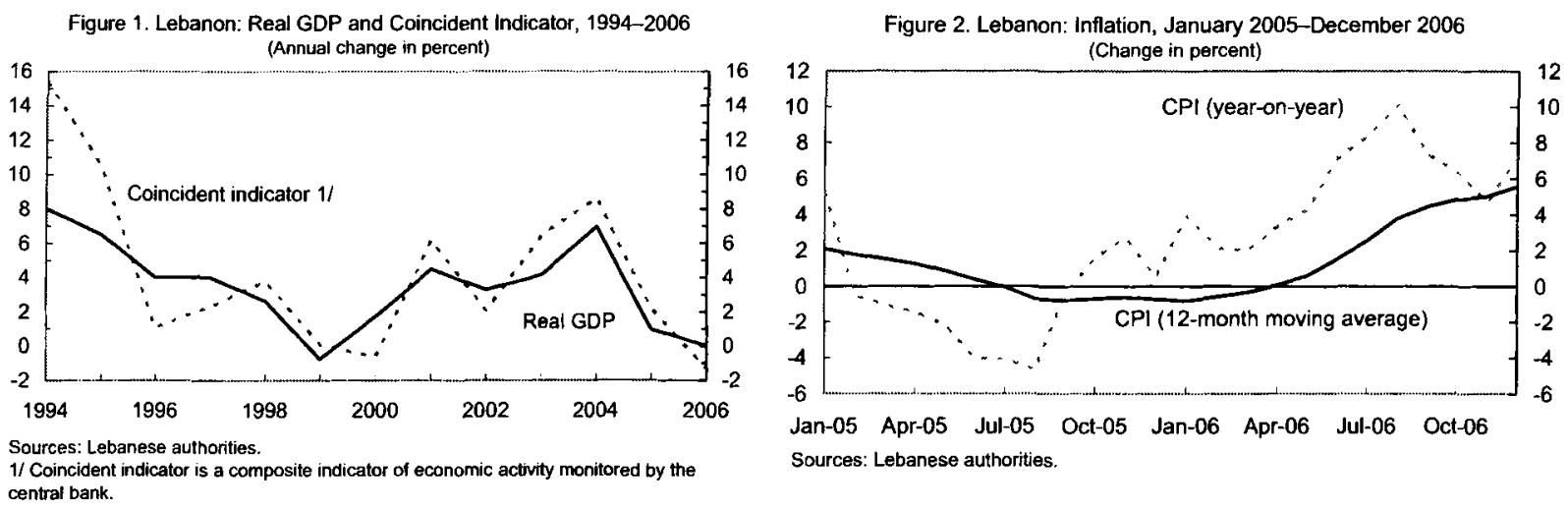

6. The conflict also pushed the primary fiscal balance (excluding grants) into a deficit of 1.2 percent of GDP in 2006 (Tables 2 and 3). Disbursed grants ( 2.9 percent of GDP) more than covered the direct budgetary impact of the conflict in 2006 (estimated at 1 percent of GDP), but a marked increase in interest expenditure (reflecting in part the expiration of some of the Paris II interest relief) caused the overall deficit to widen to 11.2 percent of GDP, from 8.5 percent in 2005 . Gross public debt rose to $\$ 40$ billion (179 percent of GDP) by end-2006 (Tables 4 and 5).

7. Financial markets weathered the conflict surprisingly well. Financial stability and confidence in the peg were maintained throughout the conflict owing to deft management of the situation by the central bank, in particular in limiting capital outflows, the banking system's strong liquidity position, and the timely deposits of $\$ 1$ billion by Saudi Arabia and $\$ 500$ million by Kuwait with the central bank. Cumulative deposit outflows of about $\$ 3$ billion during the conflict (5 percent of the deposit base) were recouped by year-end (Tables 6-8). However, deposit growth since the beginning of 2007 remains negative, deposit dollarization has not come down, and Eurobond and Credit Default Swap spreads have declined only slightly since the end of the conflict and the Paris III conference. Faced with tighter and volatile market conditions, the government had to rely on central bank financing in the second half of 2006.
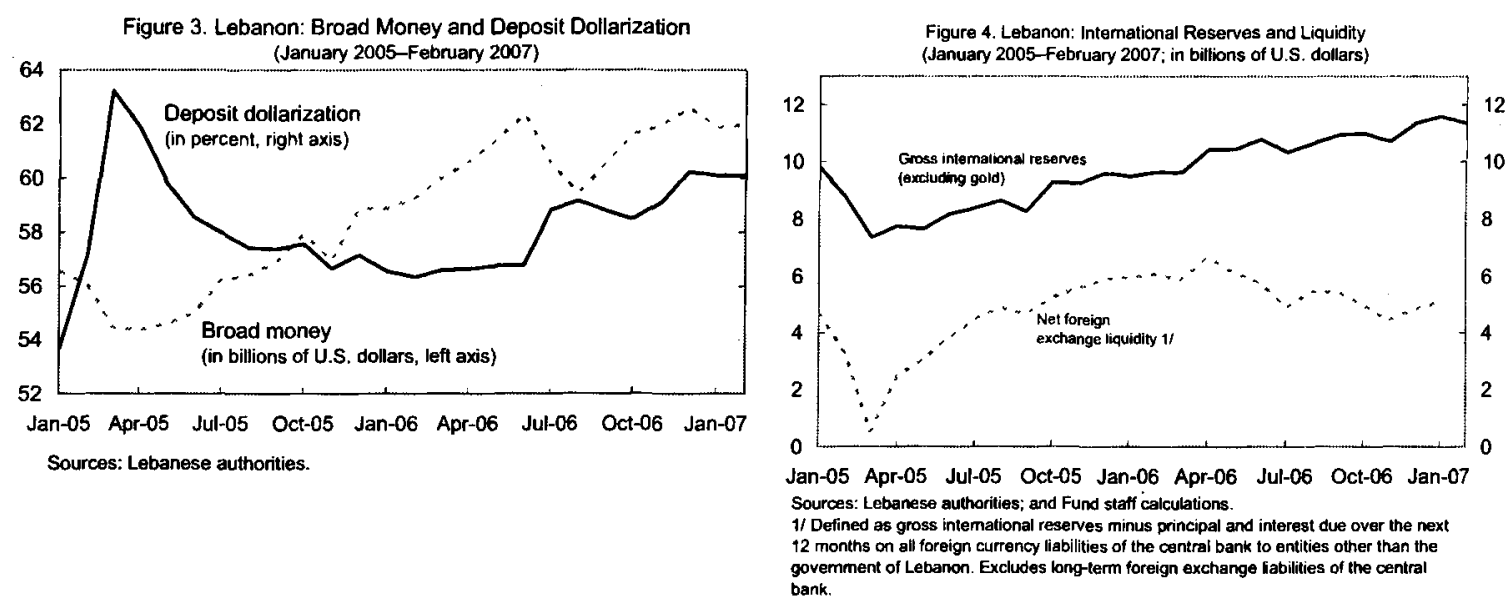

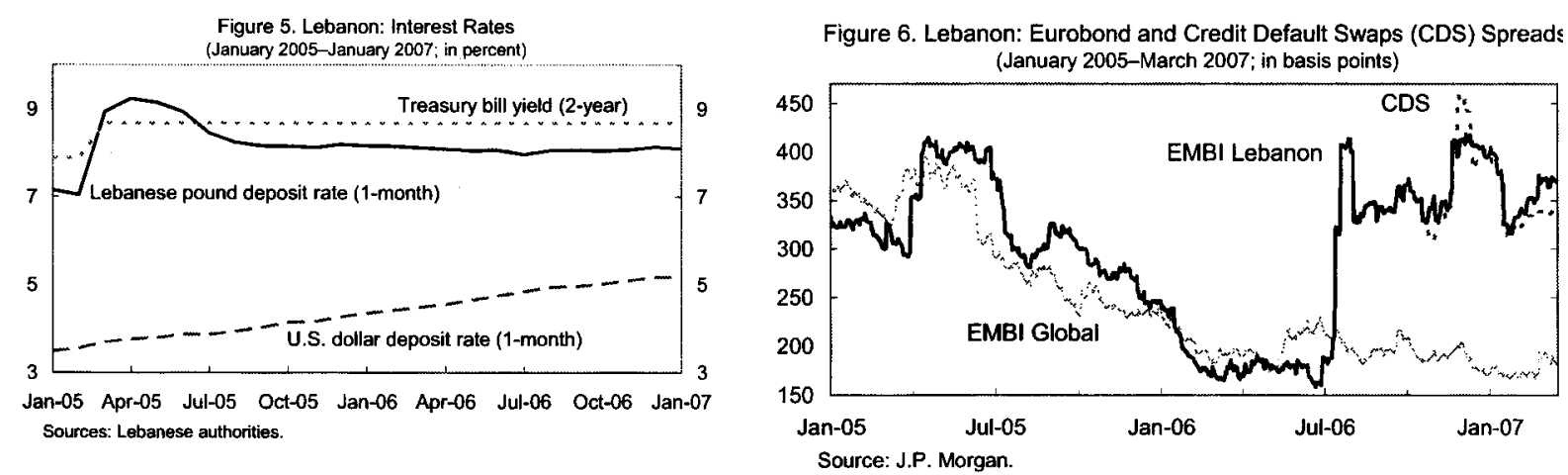

8. The overall balance of payments (including the $\$ 1.5$ billion special deposits from Saudi Arabia and Kuwait) posted a surplus of $\$ 1.7$ billion in 2006 , with gross international reserves (excluding gold) increasing to $\$ 11.4$ billion (Table 9). Trade slowed down considerably during the war and the blockade. As a result, imports were almost flat for the year as a whole, while exports still showed strong growth owing to a good first half and a recovery during the last quarter, with jewelry exports displaying particular buoyancy. Significant foreign direct investment in the first half of 2006 also helped the balance of payments. The depreciation of the U.S. dollar (to which the Lebanese pound is pegged) against major currencies more than offset the impact of domestic inflation on the real effective exchange rate, which by end-2006 depreciated modestly over December 2005.
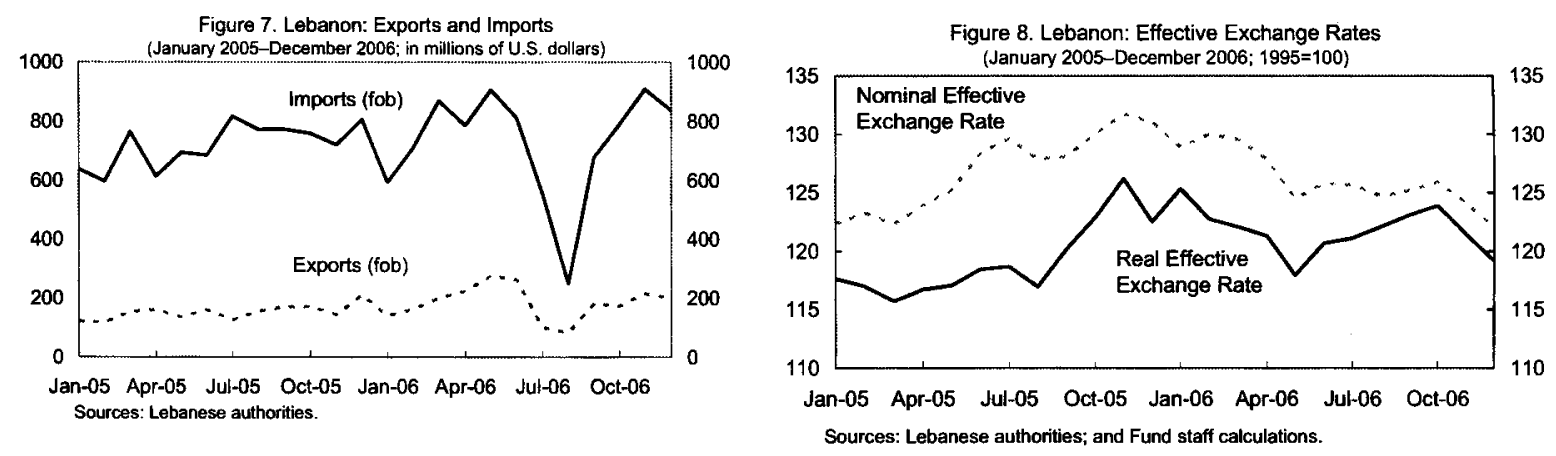

9. Based on the staff's assessment, the grant element of the $\$ 7.6$ billion pledged at Paris III that can be applied to debt reduction is estimated at around $\$ 1.4$ billion; this amount could be increased substantially depending on the flexibility shown by donors in terms of concessionality and use of funds. ${ }^{2}$ Unlike the case of Paris II, most of the support pledged at Paris III is in the form of project financing, which may be difficult to fit within the government's existing expenditure plans. To the extent that donors pursue project

\footnotetext{
${ }^{2}$ Out of total pledges of $\$ 7.6$ billion, $\$ 5.1$ billion was earmarked for the government. The rest includes money already disbursed, funds pledged to the private sector, UN agencies and NGOS, and funds previously committed.
} 
financing independently of the government's own expenditure objectives, this would add to debt. The timing of disbursement remains uncertain, although donors generally indicated that support will be phased in line with progress in the reform agenda.

\section{Policy Discussions}

10. Discussions on EPCA were based on the program presented by the authorities at Paris III donors' conference (Attachment III to the LOI). The program is broad in its objectives and range of actions (Box 2). The fiscal reform program spans five years, but owing to institutional and political constraints, as well as concerns about the state of the economy, most adjustment measures would only start in 2008. The agenda for 2007 includes a commitment to contain the deficit and initiate important structural reforms, including steps toward privatization of the two mobile phone operators, the launching of the reform plan of power utility Electricité du Liban (EdL), preparations for tax policy reforms in 2008, budget management measures, and an increase in gasoline excise revenues.

11. The authorities recognized that the volatile political climate could affect the timing of policy implementation. Some delays in reforms have occurred on account of the political stalemate, but work is under way on a number of fronts with a view to advancing the reform program as much as possible. However, given the political situation, the authorities would need to retain flexibility in choosing among structural measures to implement in case of rising tensions. For these reasons, the authorities considered it essential that the calendar of measures monitored under EPCA should focus on a limited set of clearly deliverable actions.

\section{Notwithstanding the outcome of the Paris III conference, the authorities expect}

2007 to be a very difficult year. Apart from the adverse impact of the protracted political stalemate, political tensions and uncertainty ahead of the presidential election are expected to affect adversely economic activity and private capital inflows. Based on these considerations, as well as recent economic and financial developments, the authorities and staff agreed to revise the macroeconomic assumptions for 2007 as follows:

- $\quad$ The projected rebound of GDP growth in 2007 has been lowered from 4 to 1 percent, owing to the uncertain political situation as well as the better-than-anticipated outcome for 2006. Over the medium term, growth is projected to accelerate gradually to 5 percent a year, with confidence effects and the benefits of structural reforms outweighing any dampening impact of fiscal tightening. Moreover, Lebanon is expected to benefit from continued strong regional demand for services. With the exchange rate peg, inflation is projected to return to the low single digits by end-2007, with some possible temporary up-ticks over the reform period reflecting planned increases in indirect taxes. 


\section{Box 2. Lebanon: The Authorities' Five-Year Reform Program}

The authorities' reform program covering the period 2007-11 follows closely the reform agenda developed prior to the conflict.

Fiscal adjustment and related structural reforms aim to increase the primary surplus and reduce public debt. Revenue measures include increasing the VAT rate and the tax on interest income; returning gasoline excises to their 2004 levels; introducing a global income tax to replace the current schedular system; and reforming tax administration. Expenditure measures center on containing the wage bill and other current expenditures, eliminating extrabudgetary funds, and reducing transfers by reforming the power utility and the pension system. Capital expenditures will be temporarily increased for reconstruction. The government also intends to improve the budget process and debt management.

Privatization plans include the two mobile phone operators and the fixed line telephone company. Moreover, the central bank is expected to divest ownership of Middle East Airlines and Intra (a holding company which owns Casino du Liban). Privatizations would reduce the debt, but the associated saving on debt service would be offset by the foregone profit transfers.

Monetary and exchange rate policies will focus on maintaining price stability through the exchange rate peg, relying on short-term instruments for sterilization purposes. The government will reduce its reliance on central bank financing to strengthen the independence of the Banque du Liban.

Growth-enhancing structural reforms are geared to improving the business climate and competitiveness. They will address governance problems in the public sector; streamline regulations; facilitate access to credit; enhance the enforcement of the rule of law; and deepen capital markets.

Social sector reforms seek to alleviate poverty, reduce regional income disparities, and improve education and health indicators. Specific actions will include rationalizing social spending across ministries; reforming the existing social safety net; introducing new pilot programs targeting the poor, including through cash transfers; and raising the effectiveness and coverage of health and education facilities.

- The slower than expected improvement in confidence has been reflected in higher interest rate and lower money growth projections for 2007 than envisaged at the time of Paris III. Interest rates are not projected to decline until the end of 2007, implying a possible widening of interest rate spreads vis-à-vis international dollar rates. Interest rate spreads would narrow starting in the last quarter of 2007, reflecting improved confidence and declining external financing needs. Monetary growth is projected to turn positive (but only modestly) in the second quarter, and to reach 3.5 percent by year-end. This would imply very tight 
domestic financial market conditions in 2007 . Over the medium term, monetary growth is projected conservatively at an average of 5 percent a year.

\section{A. The 2007 Fiscal Program}

\section{The $\mathbf{2 0 0 7}$ fiscal program aims at containing the primary budget deficit} (excluding grants) at 3.7 percent of GDP (indicative target), compared with 1.2 percent in 2006. The draft budget for 2007 , which has not yet been submitted to parliament (monitorable action), builds in significant reconstruction and relief spending. Excluding one-off and conflict-related spending, the primary deficit is targeted to remain roughly unchanged relative to 2006. Grant disbursements (from both Stockholm and Paris III pledges) are projected at 3.7 percent of GDP in 2007, but there is considerable uncertainty about their timing, reflecting ongoing discussions of the authorities with donors. The overall deficit (including grants) would increase from 11.2 percent of GDP in 2006 to 12.4 percent in 2007.

14. Government revenue is projected to rise by 1.1 percent of GDP in 2007 , mostly on account of an increase ( 0.7 percent of GDP) in gasoline excises. This increase will be realized by setting a floor on the gasoline excise (monitorable action), which is akin to asymmetric indexation of domestic prices to international prices. Acknowledging that their revenue projections may be on the conservative side, the authorities indicated that they intend to use any revenue over performance for debt reduction instead of increased spending. Preparations are also ongoing for the introduction of a Global Income Tax (GIT) in 2008 (with effect on 2009 tax revenues), to replace the current schedular system. The law to implement the GIT would need to be passed before end-2007.

15. Total expenditure is projected to increase by 3.1 percent of GDP relative to 2006 (3.5 percent, excluding interest). Most of the increase relative to 2006 (2.8 percent of GDP) comes from extraordinary outlays related to recovery and reconstruction. Military spending is also projected to increase due to the expansion of the army and one-off bonus payments to military personnel carried over from 2006. Additional pressures on expenditures arise from rising transfers to the public power company, EdL, the National Social Security Fund (NSSF), the Council of the South, and the Fund for the Displaced, in all, adding 1.2 percent of GDP relative to 2006. These increases are offset in part by a reduction in other spending and the postponement of infrastructure spending for the reform of EdL.

\section{Reforms intended to address the large and growing transfers to the rest of the} public sector are expected to begin in 2007. The gradual elimination of these transfers is at the core of the authorities' medium-term fiscal adjustment strategy. To that end, the authorities will develop an audit plan for the NSSF (monitorable action) in order to assess the contingent liabilities for the government, and put in place appropriate reforms. Power sector reform developed by the government in consultation with the World Bank is also set to begin in 2007. The reform will address a mix of governance and infrastructural issues, starting with 
an audit of EdL (monitorable action); however, the planned installation of remote meters to improve revenue collection has been delayed. Moreover, public expenditure reform envisages the closure in 2009 of two extrabudgetary funds - the Council for the South and the Fund for the Displaced.

\section{The authorities' program includes implementation of a public financial} management reform plan developed with technical assistance from the Fund. Weak controls and accountability have prevented effective budgetary control, making it difficult to adjust line ministries' spending in line with the government's priorities. One key concern for the ministry of finance has been the ability of line ministries to roll over expenditures from one budget year to the next. The authorities committed to eliminating, in the 2007 budget, the rollover of any spending that has not already been contracted (monitorable action), and to prepare the necessary measures to prevent the unchecked carry over of expenditures starting with the 2008 budget. They noted that they had already reduced the outstanding stock of committed carryovers from around 5.7 percent of GDP in early 2005 to around 1.5 percent at the end of 2006. The authorities have also completed the automation of payments, which is a prerequisite for the planned move to a Treasury Single Account.

18. Despite the large overall deficit, gross government debt is projected to decline in 2007 by three percentage points of GDP to 176 percent of GDP, reflecting a \$1.6 billion (6.7 percent of GDP) transfer to the government from the (unrealized) valuation gains of gold held by the central bank. Lebanese law provides for the annual transfer of 80 percent of gold revaluation, although an informal agreement between the ministry of finance and the central bank had suspended such transfers for three years. The authorities indicated that the transfer would be used to retire government debt held by the central bank, and would therefore have no direct monetary impact. The projected decline in gross debt matches that of net debt (indicative target).

\section{The financing of the deficit is expected to take place largely through donor} financing and borrowing from commercial banks. The program projects that official financing (Paris III and undisbursed Stockholm pledges) would cover $\$ 2.7$ billion of the $\$ 3.8$ billion deficit (before grants), and that commercial banks would provide another $\$ 900$ million. The remaining $\$ 200$ million would be covered by domestic public institutions and the central bank. In gross terms, the financing requirement of the government in 2007 is projected at $\$ 10$ billion (43 percent of GDP), \$3 billion of which is in foreign exchange. Most of the debt is held by domestic banks and their ability to refinance the government depends in large part on the strength of deposit growth. The authorities considered that, in the current uncertain market environment, there was little scope for increasing private financing by raising interest rates. Therefore, in the absence of a pick up in monetary growth, or in the event of a shortfall in donor financing, the authorities noted that they would have to resort to additional central bank financing of the government (indicative target), with a direct impact on international reserves. While committed to the quantitative indicative targets of the program, the authorities argued therefore that the target on central bank credit to the 
government allow for a degree of flexibility should they face difficulties in borrowing from the market or shortfalls in donor financing. They agreed that they would consult with staff should it become necessary to exercise such flexibility.

\section{B. Monetary and Exchange Rate Policy}

\section{To protect the exchange rate peg and financial stability in the face of large} liquidity shocks, the banking sector (central bank and commercial banks) has maintained a high level of liquid foreign assets. The program aims at maintaining an average level of gross international reserves (indicative target $t^{3}$ ) equivalent to roughly 20 percent of total deposits, which has proven adequate in the face of the large financial shocks of 2005 and $2006 .{ }^{4}$ Nevertheless, given the difficult financial outlook expected in 2007 , and the low projected rate of monetary growth, the authorities considered that some temporary decline in gross reserves target was justified to enable them to address possible intra-annual pressures, with the objective of returning by end-year to the level of international reserves projected for end-March 2007. Consistent with the international reserves objective, the authorities will also seek to contain the growth of central bank claims on the government (indicative target), with the understanding that temporary shortfalls in market or donor financing would have to be accommodated through additional central bank financing. ${ }^{5}$

\section{The central bank will continue to monitor closely liquidity conditions in the} domestic markets because of its impact on international reserves. In managing liquidity, the authorities confirmed their objective of moving to a more conventional system of short-term monetary instruments, but indicated that it would be too risky to reform monetary policy instruments in the current unstable market environment. They were of the view that, in times of high uncertainty, the effectiveness of interventions in the money market in countering pressures on the exchange rate was limited, and that they would have to continue to rely on longer-term monetary instruments in order to prevent banks from liquidating at short notice their claims on the central bank.

\section{The costs of past central bank operation constrain liquidity management. As a} result of such operations (including the cost of defending the peg and maintaining international reserves), the central bank's balance sheet generates large cash losses. Given the

\footnotetext{
${ }^{3}$ For the purpose of the program, gross reserves are defined to include Lebanon Republic Eurobonds in order to align the headline number to the national definition. However, the floor on gross reserves is adjusted for any change in the holdings of Eurobonds.

${ }^{4}$ Table 12 shows financial and external vulnerability indicators.

${ }^{5}$ The target ceiling includes the redemption of LL2,380 billion in Treasury bills held by the central bank to be paid through the transfer of the gold revaluation gains.
} 
expected slow recovery of money demand growth in 2007, the liquidity injection stemming from these losses is likely to have a direct impact on international reserves. The monetary authorities assured staff that they would monitor the situation closely, with a view to avoiding a structural imbalance between their liquidity injections and money demand.

\section{Structural Reforms}

\section{EPCA discussions on structural reforms focused on the following macrocritical} areas:

- Privatization of the telecom sector. The authorities confirmed that the board of the Telecom Regulatory Authority (TRA) was now in place. Another precondition for the privatization of the sector is passage of a law enabling the sale of the assets of the mobile phone operators and relevant operating licenses. The authorities plan to submit such legislation to parliament by end-June 2007 (monitorable action). Because of the political calendar, the authorities expect to launch the privatization process by sending invitations for expression of interest in the purchase of the two operators toward the end of the year (monitorable action). The two companies are currently privately managed under a contract that expires in April 2008, which would therefore permit completing the transfer of the assets and licenses to the new owners by mid2008. The privatization would be for the full value of the assets and the licenses, compared with the assumption of a partial privatization in the Paris III program. ${ }^{6}$ The privatization of the fixed line, along with a third mobile license, is projected to begin in 2008 , with a first partial sale to a strategic investor.

- $\quad$ Power sector. The reform of EdL includes actions on both governance and infrastructure. On the governance front, there is a need to both clarify the financial situation of the company (the last audited financial accounts date to 2001) and corporatize it so that it can operate more independently and according to commercial principles. On the infrastructure front, investment is needed to install remote meters to reduce the problem of non-technical losses, and to upgrade production facilities (possibly through Build Operate and Transfer or other financing arrangements). The World Bank will take the lead in this area and has begun discussions on a power sector lending operation. Preparation of an audit plan by end-June (monitorable action) will serve as an input to this reform.

- Social security, pension reform and health care reform. Two of the three funds of the NSSF (the family allowance fund and the health insurance fund) run structural deficits which give rise to large liabilities for the government, which has a legal obligation to cover some of the costs of NSSF. Inadequate contribution rates,

\footnotetext{
${ }^{6}$ The larger upfront reduction in the debt to GDP ratio is offset by a lower primary surplus over the medium-term due to the foregone profit transfers from the telecom operators.
} 
excessive and unchecked benefit payments, and other possible leakages may be at the origin of these losses. However, in the absence of a clear financial picture, reforms have been delayed and the government has been reluctant to meet all of the financial requests of NSSF. ${ }^{7}$ The private pension system (the third fund in NSSF) has been running surpluses, but a reform of the system from an end-of-service lump sum payment scheme to a fully-funded pension system may entail large transitional costs for the government. The World Bank is directly involved in these areas. The preparation of an audit plan by end-June (monitorable action) will facilitate the reform process.

\section{The Medium-Term Fiscal Program and Debt Sustainability Analysis}

\section{Based on the revisions to the $\mathbf{2 0 0 7}$ fiscal projections and the macroeconomic} framework, the staff's medium-term scenario projects that the implementation of the authorities' program would raise the primary surplus to $5 \frac{1}{2}$ percent of GDP by 2011-12. Relative to the authorities' Paris III projections, the staff's scenario takes a more conservative view on the fiscal dividend from the reforms of the electricity company and the pension system and factors in a larger loss in nontax revenue due to the full rather than partial privatization of the mobile phone operators. The current scenario also embodies more recent (and higher) oil price assumptions over the medium term, which translates into higher projected losses of EdL and related budgetary transfers. However, the assumed rate of real GDP growth is considerably higher than the historical average.

\section{The medium-term fiscal adjustment is about equally divided between revenue} and expenditure measures (see table below). The first step of the VAT rate increase, an increase in the interest income withholding tax, and the introduction of the GIT are all planned for 2008. In the event of delays in the implementation of any of these measures, the authorities intend to take compensatory actions to match the original adjustment path of Paris III.

\section{Under the scenario, the government debt ratio would decline to 128 percent} of GDP by 2012. Of the 51 percentage point net reduction in the debt ratio over the period 2007-12, 31 points would come from privatization, 10 points from fiscal adjustment, 7 points from the 2007 transfer of gold revaluation gains, 6 points from Paris III contributions, and 3 points from post war recovery grants, with the automatic debt dynamics partly offsetting these gains. Fiscal adjustment would progressively become the main driving factor behind debt reduction and, by 2012 , the projected primary surplus would exceed the debt-stabilizing primary surplus by 6 percent of GDP. The debt ratio would thus remain on a downward path even with some relaxation of the fiscal stance after 2012. This is illustrated in the Debt

\footnotetext{
${ }^{7}$ NSSF has covered the residual financing needs of the two funds from its reserves, and by running arrears.
} 
Sustainability Analysis (DSA), which is carried out over a 20-year horizon and assumes that the primary surplus returns to 3 percent of GDP by 2015 (Figure 9 and Table 10).

\begin{tabular}{|c|c|c|c|c|c|c|c|}
\hline & 2007 & 2008 & 2009 & 2010 & 2011 & 2012 & Cumulative $1 /$ \\
\hline Total primary fiscal effort & 0.1 & 2.1 & 3.4 & 3.1 & 1.0 & 0.1 & 9.7 \\
\hline Revenue & 0.9 & 2.0 & 0.2 & 1.8 & 0.5 & -0.1 & 5.3 \\
\hline Increase in the VAT rate (from 10 to 12 , and then to 15 percent) & 0.0 & 1.2 & 0.0 & 1.3 & 0.0 & 0.0 & 2.5 \\
\hline Global income tax & 0.0 & 0.0 & 0.4 & 0.3 & 0.3 & 0.0 & 1.0 \\
\hline Increase in gasoline excises to their pre-capping level & 0.7 & 0.1 & 0.2 & 0.1 & 0.1 & -0.1 & 1.1 \\
\hline Other $/ 2$ & 0.2 & 0.7 & -0.3 & 0.0 & 0.1 & 0.0 & 0.7 \\
\hline Expenditure & -0.8 & 0.1 & 3.1 & 1.3 & 0.5 & 0.2 & 4.4 \\
\hline Wages (reforms leading to a nominal freeze of the wage bill) & -0.2 & 0.4 & 0.3 & 0.3 & 0.3 & 0.1 & 1.1 \\
\hline Reduction in EdL losses & -0.7 & -0.2 & 2.4 & 0.1 & 0.1 & 0.1 & 1.9 \\
\hline Other $3 /$ & 0.1 & -0.1 & 0.4 & 0.8 & 0.1 & 0.0 & 1.4 \\
\hline Adjustments & -1.8 & -1.0 & -1.7 & -0.6 & -0.8 & 0.0 & -5.9 \\
\hline Revenue loss due to privatization & 0.0 & -1.4 & -1.9 & 0.0 & 0.0 & 0.0 & -3.4 \\
\hline Grants (Stockholm and Paris III) & 0.8 & -1.5 & -1.1 & -0.6 & -0.4 & 0.0 & -3.0 \\
\hline Conflict-related expenditure & -2.8 & 2.3 & 1.5 & 0.0 & 0.0 & 0.0 & 1.0 \\
\hline Other $4 /$ & 0.2 & -0.3 & -0.2 & 0.0 & -0.3 & 0.0 & -0.6 \\
\hline Change in primary surplus & -1.7 & 1.1 & 1.7 & 2.5 & 0.2 & 0.1 & 3.8 \\
\hline \multicolumn{8}{|c|}{$\begin{array}{l}\text { Source: Fund staff projections. Figures may not add due to rounding. } \\
1 / \text { The cumulative adjustment starls from } 2006 \text {. } \\
2 / \text { Includes (i) increasing the tax rate on interest income from } 5 \text { to } 7 \text { percent; (ii) improving non-tax revenue from govemment properties; (iii) taxing } \\
\text { illegal seashore properties and improving property tax administration; and (iv) improved buoyancy of "Other Treasury revenue" to return to pre- } \\
\text { conflict levels. }\end{array}$} \\
\hline $\begin{array}{l}3 / \text { Includes (i) containment of other current expenditures; and (ii) increa } \\
\text { Review). } \\
4 / \text { Includes the fiscal impact of exogenous factors and one-off effects. }\end{array}$ & I capital & ending & advo & & Vorle & 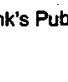 & Expenditure \\
\hline
\end{tabular}

27. The DSA reveals that under a number of macroeconomic shocks, the debt-to-GDP ratio would revert to an unsustainable path. Box 3 describes the shocks in detail. The implication is that additional grant financing and/or stronger fiscal adjustment would be needed over the medium term to place the debt ratio on a firmer path toward sustainability.

\section{E. The Paris III Package and Commercial Banks' Contribution}

28. Discussions are under way with all Paris III donors to finalize the terms of the package of financial assistance and the conditions for its disbursement. The medium-term scenario builds in the assumption that official budgetary assistance (Stockholm and Paris III) over 2007-12 will come to $\$ 1.9$ billion in grants, and $\$ 2.6$ billion in loans. ${ }^{8}$ The authorities indicated that, in view of the debt reduction objective, they would seek flexibility from donors to convert Paris III project assistance pledges into budgetary support on highly concessional terms, so as to increase the overall contribution of Paris III to debt reduction.

\footnotetext{
${ }^{8}$ The Paris III package is assumed to contain $\$ 822$ million in grants and $\$ 2.4$ billion in soft loans for budget support, with an average grant element of 23 percent.
} 
Figure 9. Lebanon: Public Debt Sustainability

(Public debt in percent of GDP)

Panel 1: Program and Historical GDP Growth Scenario 1/

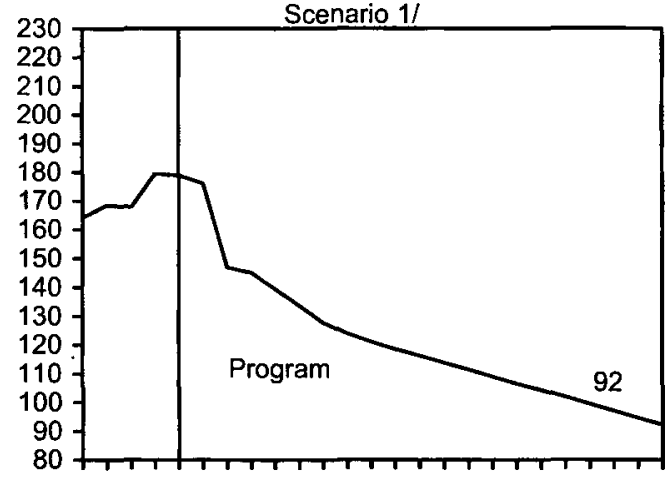

200220052008201120142017202020232026

Panel 3: Growth Shock 1/ (In percent per year)

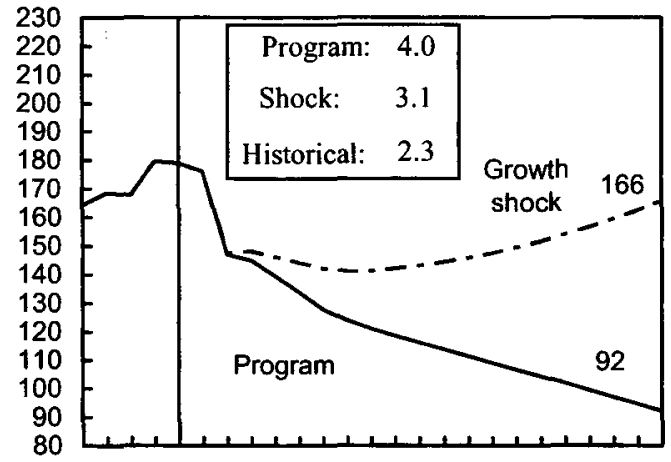

200220052008201120142017202020232026

Panel 5: Combined Shock 3/

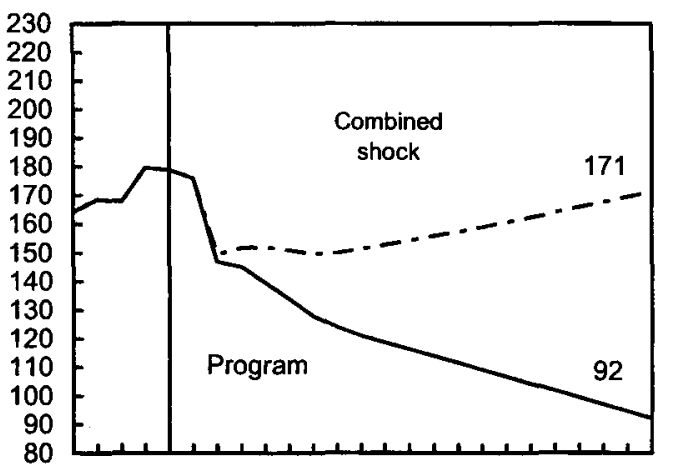

200220052008201120142017202020232026
Panel 2: Real interest Rate Shock 1/ (In percent)

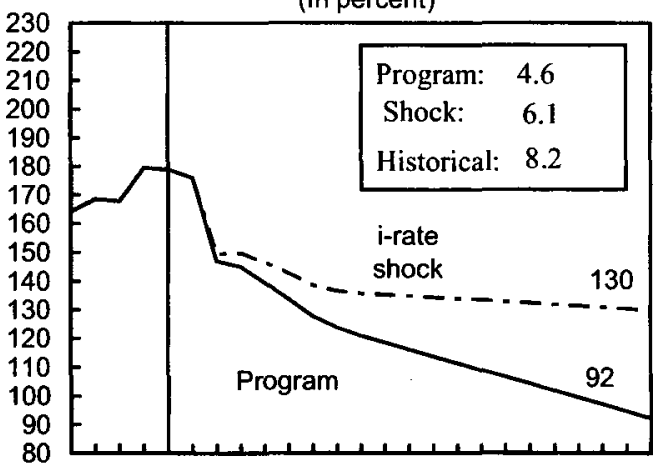

200220052008201120142017202020232026

Panel 4: Primary Balance Shock 2 I (In percent of GDP)

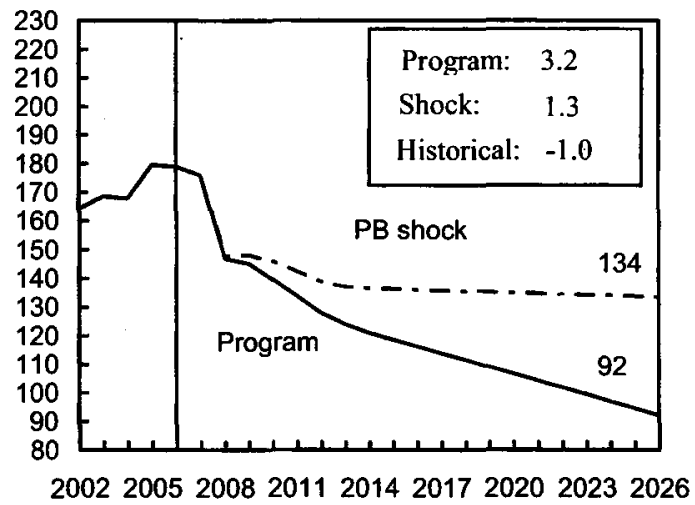

Panel 6: No Privatization 4!

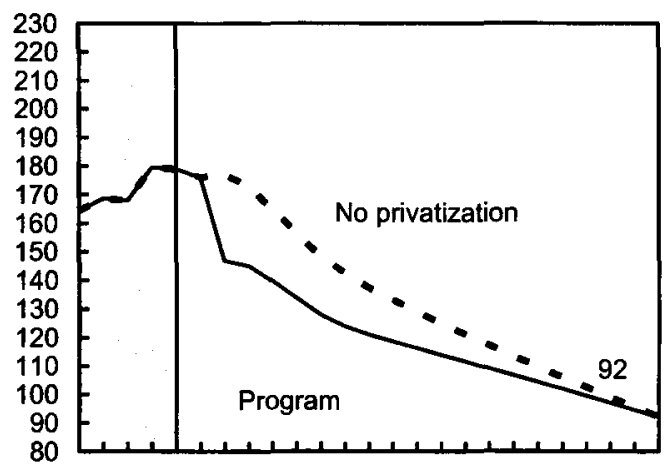

200220052008201120142017202020232026

Sources: International Monetary Fund, Country desk data, and staff estimates.

$1 /$ Growth shock based on average 2002-2006 values. Interest rate shock is permanent one-half standard deviation shock. Historical path is defined in Table 14, Footnote 8. Figures in the boxes represent average projections for the respective variables in the program scenario and shock scenario; historical refers to 10 year averages.

From 2012, the primary fiscal surplus reverts slowly to a long term level of 3 percent of GDP.

$2 /$ Through 2012, the planned revenue and expenditures reforms generate half of their estimated yield in the scenario; after 2012, the primary surplus slowly declines to 1 percent of GDP.

$3 /$ Combined real interest rate and growth rate ( 50 percent intensity), and primary balance shock (100 percent intensity).

4/ No privatization in the projected period. 
Box 3. Lebanon: Shock Scenarios for the Debt Sustainability Analysis

Panel 1: the program scenario.

Panel 2: a permanent increase in the real interest rate by 150 basis points (i.e., one-half standard deviation from its past distribution) relative to the program scenario.

Panel 3: a drop in average GDP growth from 4.1 percent in the program scenario to 3.1 percent, i.e., the average rate of $2002-06$.

Panel 4: a shortfall in the yield from fiscal reforms, i.e., halving of the yield of the fiscal reform package while meeting in full reconstruction spending (this reduces the average primary surplus to 1.6 percent of GDP in 2007-12, compared with 4.4 percent under the program), and convergence of the primary surplus to 1 percent of GDP in the long run (compared with 3 percent in the program scenario).

Panel 5: a combination of the fiscal shock with 50 percent of the growth and interest rate shocks.

Panel 6: no privatization-since privatization essentially brings forward the income stream from the privatized companies, the scenarios with and without privatization converge to the same point in the long run.

29. The authorities are also discussing a possible contribution from the local commercial banks. The proposal envisages a voluntary subscription to zero (or low) interest Treasury bills to the tune of 5-10 percent of the commercial banks' deposit base. The contribution would be staggered over five years and conditioned on the government's observance of its deficit target - the EPCA target would be the trigger for this year. This would ensure that the contribution is matched by a reduction in the sovereign risk which banks carry on their balance sheets.

\section{F. Access, Program Monitoring, and Capacity to Repay the Fund}

- Staff considers that access of 25 percent of quota would be justified in light of Lebanon's large external financing needs in relation to its quota.

- Staff will update the Executive Board (and donors) regularly, through informal country matters sessions, on progress under EPCA, including on performance relative to the quarterly indicative targets and monitorable actions set out in the LOI.

- A safeguard assessment of the Banque du Liban has been initiated.

- Lebanon is expected to meet its financial obligations to the Fund in a timely manner. The authorities note that, to date, Lebanon has maintained a perfect record of meeting external debt payments, even during its civil war and recent episodes of financial pressures. Fund credit would be low relative to quota and in absolute terms, and debt 
service to the Fund would remain below 0.2 percent of exports of goods and non-factor services (Table 11).

- The provision of Fund financing to Lebanon under the existing circumstances poses risks to the Fund, owing to Lebanon's very high debt ratio. Financing committed by certain donors that could help to reduce Lebanon's external financing requirements, while a very welcome indication of support, would not be sufficient to significantly alter the debt situation. Against this background, staff and management have consulted with the authorities and, through the relevant Executive Directors, with the bulk of Lebanon's official bilateral donors and creditors to address the risks that are posed by the provision of Fund financing. Based on these consultations, Management has understood that these members confirm the Fund's preferred creditor status in respect of drawing by Lebanon under the EPCA and, similarly, that they acknowledge the importance of timely repayment to the Fund of the amounts provided under the EPCA, in accordance with the Fund's preferred creditor status.

- The main risk to program implementation stems from the ongoing domestic and regional political tensions. The government is committed to its economic program and determined to move ahead with reforms swiftly. However, the continuation of the political stalemate and a possible further escalation in the run-up to the presidential elections in the Fall of 2007 could delay or derail implementation of specific measures.

\section{STAFF APPRAISAL}

30. Seven months after the end of the conflict with Israel, economic and financial conditions in Lebanon remain very difficult. Market confidence is hostage to the domestic political situation, and structural imbalances and vulnerabilities (chief among them the unsustainable debt burden) have been magnified by the economic and financial situation. Against this environment, the authorities face the difficult simultaneous challenges of economic recovery, reform, and fiscal adjustment.

31. The deferment of adjustment measures until 2008 and beyond, although motivated by the economic fallout from the conflict and the need to support economic recovery and reconstruction, has increased significantly the government's financing needs at a time when market access has become more difficult. Although 2007 should be viewed as a transition year, every opportunity should therefore be seized to advance the reform and adjustment process, particularly since tensions related to the financing of the fiscal program will remain a key risk factor in 2007. The program is build on conservative budget revenue projections, and staff welcomes the authorities' commitment to use any over performance on revenue to reduce the deficit and not to increase spending.

32. The success of the program rests crucially on donors matching the authorities' own efforts with timely disbursement of their pledges. Moreover, there is a need for donor 
support to be aligned with the authorities' macroeconomic objectives and policy priorities. In this regard, much of the project financing pledges at Paris III will have limited benefits if not channeled flexibly to the government in the form of outright budget support or by financing specific line items of the budget. An enhancement of the concessionality element of the overall package is also needed to address the debt sustainability problem.

33. Although post-conflict conditions preclude much fiscal adjustment in 2007, it is essential to set up the stage for meaningful and sizable fiscal adjustment in 2008 , while improving the quality of spending. The 2007 program envisages yet another increase in transfers to the power sector offset by a compression of more productive infrastructure spending. Structural reforms need to be initiated urgently to address deep-seated spending rigidities, eliminate the burden of large inefficient transfers, and reduce the risks from contingent liabilities. In particular, setting in motion the reform of EdL, the private pension system and the health sector-all in cooperation with the World Bank-should be key objectives for 2007 . The planned audit of the social security fund will also be critical to clarify the financial obligations of the state vis-à-vis the fund and address the build up of contingent liabilities. The unsustainable pension liabilities of the government to civil servants and military personnel also deserve urgent attention, as they constitute a major risk for the budget which could wipe out any gains made in reducing power sector and social security transfers. On the revenue front, the planned introduction of the GIT in 2008 remains an important objective, not only because of its positive budgetary implications, but also as a way to distribute more equitably the burden of adjustment. To that end, the authorities need to move swiftly to pass the relevant legislation before the beginning of 2008 .

\section{Improvements in public financial management are equally important to the} achievement of the Paris III objectives. Insufficient budgetary controls make it very difficult to align government spending to the government's policy priorities. This is evident in the social sector, where the fiscal space required for the government's reform agenda can only be generated by clawing back from low priority and less efficient spending. The public financial management reform agenda developed with the assistance of Fund staff offers a useful road map, and it would be important that its recommendations be reflected in the 2008 budget.

35. The projected reduction of the debt ratio in 2007 is the direct result of the gold revaluation transfer from the central bank to the government. The monetary impact is neutralized by the government's decision to use the proceeds to retire debt held by the central bank, rather than reduce its market financing. However, while this operation reduces the headline debt figure, it does not alter the underlying net worth of the state. Moreover, the central bank will suffer a loss of income owing to the foregone interest earnings on T-bills.

36. In the event of renewed pressures, defending the peg and maintaining market confidence in this transition year will again require a mix of foreign exchange intervention and interest rate increases. In particular, the instruments deployed in 2005 
and 2006 to contain pressures on reserves could be reactivated in case of need (i.e., discounting of domestic currency paper against long-term central bank dollar paper). At the same time, as noted in the Paris III document, the monetary authorities should prepare the institutional ground for introducing more traditional short-term monetary instruments. This will also help strengthen the balance sheet of the central bank and reduce its cash losses.

\section{There is considerable scope for enhancing coordination between the central} bank and the government. Procedures are not in place for the regular exchange of information and policy planning, and the problems for policymakers are complicated by the absence of clear market signals due to the absence of efficient primary and secondary financial markets. Progress on a number of plans, including the establishment of a primary dealer market for T-bills and of the Higher Debt Council to coordinate debt management issues, would be important in filling these institutional gaps.

\section{In the current domestic and regional political environment, Lebanon remains} highly vulnerable to swings in confidence. The international reserve buffer and expected donor inflows should mitigate the risks to financial stability, but the increase in the government's financial imbalance in 2007, even if temporary, poses additional strains on the system. Faced with tight market conditions and a possible shortfalls in donor financing, the authorities may have to resort to additional central bank financing of the government and accept the related loss of international reserves. Staff welcomes the authorities' intention to consult with staff in such circumstances, and agrees with the authorities that the target on central bank financing of the government should be viewed with flexibility. Additional recourse to central bank financing would be a move in the wrong direction. Therefore, the staff emphasize the importance of donor financing being disbursed as scheduled. However, the authorities should also be ready to accept higher interest rates in response to a worsening of market conditions. Persistent market pressures may also need to be met by accelerating the pace of fiscal adjustment.

39. In all, the program is built on the expectation that 2007 will be a difficult year, and that the authorities' medium-term objectives remain subject to significant risks. As illustrated by the DSA, the large debt overhang will remain a threat to financial stability well into the medium term, and any delay in the implementation of the structural reform agenda in 2007 will have consequences for the pace and the quality of fiscal adjustment in 2008 and beyond. In this context, EPCA provides support for the transitional challenges of moving from a post-conflict situation to a program of ambitious fiscal adjustment starting in 2008. Timely disbursement of donor support is key, particularly in a situation where market financing may be adversely affected by the political stalemate. However, market sentiment as well as donor support are themselves dependent on the authorities' ability to deliver on their reform commitments. The staff considers that the criteria for Fund support under EPCA have been met and therefore recommends that the Board approve the authorities' request. 
Table 1. Lebanon: Selected Economic Indicators, 2003-12

\begin{tabular}{|c|c|c|c|c|c|c|c|c|c|c|}
\hline & $\begin{array}{c}2003 \\
\text { Act. }\end{array}$ & $\begin{array}{c}2004 \\
\text { Act. }\end{array}$ & $\begin{array}{r}2005 \\
\text { Act. }\end{array}$ & $\begin{array}{r}2006 \\
\text { Prel. Act. }\end{array}$ & $\begin{array}{l}2007 \\
\text { Proj. }\end{array}$ & $\begin{array}{c}2008 \\
\text { Proj. }\end{array}$ & $\begin{array}{l}2009 \\
\text { Proj. }\end{array}$ & $\begin{array}{l}2010 \\
\text { Proj. }\end{array}$ & $\begin{array}{l}2011 \\
\text { Proj. }\end{array}$ & $\begin{array}{l}2012 \\
\text { Proj. }\end{array}$ \\
\hline Output and prices & \multicolumn{10}{|c|}{ (Annual percentage change) } \\
\hline Real GDP (market price) & 4.1 & 7.0 & 1.0 & 0.0 & 1.0 & 3.5 & 4.5 & 5.0 & 5.0 & 5.0 \\
\hline Consumer prices (end of period) & 2.2 & 2.0 & 0.5 & 7.2 & 2.0 & 3.0 & 2.0 & 3.5 & 2.0 & 2.0 \\
\hline Consumer prices (period average) & 1.3 & 1.7 & -0.7 & 5.6 & 3.5 & 2.5 & 2.5 & 2.8 & 2.8 & 2.0 \\
\hline Investment and saving & \multicolumn{10}{|c|}{ (In percent of GDP) } \\
\hline Gross capital formation & 19.3 & 22.1 & 17.0 & 12.2 & 16.6 & 19.9 & 21.6 & 21.2 & 20.8 & 20.7 \\
\hline Government & 3.1 & 3.3 & 2.2 & 2.5 & 5.0 & 3.0 & 2.7 & 2.8 & 2.9 & 2.9 \\
\hline Non-government & 16.2 & 18.9 & 14.9 & 9.7 & 11.7 & 16.9 & 18.9 & 18.4 & 17.9 & 17.8 \\
\hline Gross national savings & 6.0 & 6.3 & 5.3 & 5.3 & 5.6 & 9.9 & 12.2 & 14.6 & 15.1 & 15.3 \\
\hline Government & -10.2 & -5.4 & -6.3 & -8.6 & -7.4 & -7.7 & -4.2 & -0.9 & -0.3 & 0.2 \\
\hline Non-government & 16.3 & 11.7 & 11.6 & 14.0 & 13.1 & 17.5 & 16.4 & 15.5 & 15.4 & 15.1 \\
\hline Public finances & \multicolumn{10}{|c|}{ (In percent of GDP) } \\
\hline Revenue (including grants) & 22.1 & 23.2 & 22.9 & 24.9 & 26.7 & 25.6 & 22.6 & 23.8 & 23.5 & 23.4 \\
\hline Expenditure & 35.4 & 31.9 & 31.4 & 36.0 & 39.1 & 36.2 & 29.6 & 27.6 & 26.7 & 26.1 \\
\hline Budget balance (including grants) & -13.3 & -8.7 & -8.5 & -11.2 & -12.4 & -10.6 & -6.9 & -3.7 & -3.2 & -2.7 \\
\hline Primary balance (including grants) & 3.3 & 3.5 & 2.1 & 1.7 & 0.0 & 1.1 & 2.8 & 5.2 & 5.4 & 5.5 \\
\hline Total government debt & 169 & 168 & 180 & 179 & 176 & 147 & 145 & 140 & 134 & 128 \\
\hline Monetary sector & \multicolumn{10}{|c|}{ (Annual percentage change, unless otherwise indicated) } \\
\hline Credit to the private sector & 0.3 & 5.2 & 1.9 & 6.0 & 5.0 & 27.5 & 6.0 & 6.0 & 6.0 & 6.0 \\
\hline Base money & 12.3 & 10.3 & 4.7 & 8.2 & 4.4 & 8.4 & 4.8 & 5.9 & 5.9 & 5.8 \\
\hline Broad money $1 /$ & 15.5 & 12.3 & 3.5 & 6.4 & 3.5 & 7.0 & 4.0 & 5.0 & 5.0 & 5.0 \\
\hline Velocity of broad money (level) & 0.4 & 0.4 & 0.4 & 0.4 & 0.4 & 0.4 & 0.4 & 0.4 & 0.4 & 0.4 \\
\hline \multicolumn{11}{|l|}{ Interest rates (period average, in percent) } \\
\hline Three-month treasury bill rate & 6.7 & 5.2 & 5.2 & 5.2 & $\ldots$ & $\ldots$ & $\ldots$ & $\ldots$ & $\ldots$ & $\ldots$ \\
\hline Two-year treasury bill rate & 8.0 & 7.9 & 8.5 & 8.7 & 8.2 & 7.6 & 7.4 & 7.3 & 7.1 & 7.1 \\
\hline External sector & \multicolumn{10}{|c|}{ (In percent of GDP, unless otherwise indicated) } \\
\hline Exports of goods (in US\$, percentage change) & 43.2 & 18.3 & 11.1 & 22.5 & 12.9 & 14.6 & 14.0 & 15.0 & 14.5 & 13.6 \\
\hline Imports of goods (in US $\$$, percentage change) & 10.4 & 30.3 & -1.2 & 1.8 & 17.5 & 4.3 & 9.7 & 4.3 & 8.7 & 9.2 \\
\hline Merchandise trade balance & -24.2 & -30.2 & -28.6 & -25.4 & -29.2 & -27.5 & -27.7 & -25.5 & -24.9 & -24.7 \\
\hline Current account excluding official transfers & -13.9 & -16.3 & -12.4 & -11.6 & -15.3 & -10.6 & -9.9 & -7.1 & -6.2 & -5.8 \\
\hline Current account including official transfers & -13.3 & -15.8 & -11.7 & -6.8 & -11.0 & -10.0 & -9.4 & -6.6 & -5.7 & -5.4 \\
\hline Foreign direct investment & 8.7 & 10.9 & 12.3 & 12.0 & 7.6 & 23.4 & 7.9 & 8.1 & 8.2 & 8.3 \\
\hline Total external debt & 175 & 188 & 191 & 200 & 183 & 175 & 163 & 155 & 148 & 140 \\
\hline Gross reserves (in millions of U.S. dollars) & 10,271 & 9,575 & 9,611 & 11,353 & 10,440 & 13,139 & 11,668 & 12,215 & 13,054 & 14,030 \\
\hline In months of next year imports of goods and services & 8.1 & 7.7 & 7.3 & 7.6 & 6.7 & 7.7 & 6.5 & 6.3 & 6.2 & 6.7 \\
\hline In percent of short-term external debt $2 /$ & 35.6 & 27.1 & 28.4 & 31.9 & 32.5 & 40.1 & 35.9 & 36.5 & 37.1 & 40.5 \\
\hline In percent of banking system foreign currency deposits & 40.3 & 31.9 & 29.0 & 30.1 & 26.7 & 31.2 & 26.8 & 26.8 & 27.4 & 28.2 \\
\hline In percent of total banking system deposits & 24.8 & 21.0 & 20.1 & 21.9 & 19.3 & 22.4 & 19.0 & 18.8 & 19.0 & 19.3 \\
\hline \multicolumn{11}{|l|}{ Memorandum items: } \\
\hline Nominal GDP (in billions of U.S. dollars) & 19.8 & 21.4 & 21.4 & 22.6 & 23.6 & 25.0 & 26.6 & 28.5 & 30.5 & 32.7 \\
\hline Net imports of petroleum products (millions of U.S. dollars) & $-1,057$ & $-1,833$ & $-2,082$ & $-2,172$ & $-2,072$ & $-2,286$ & $-2,380$ & $-2,489$ & $-2,593$ & $-2,702$ \\
\hline Local currency per U.S. dollar (period average) & 1,508 & 1,508 & 1,508 & 1,508 & $\ldots$ & $\ldots$ & $\ldots$ & $\ldots$ & $\cdots$ & .. \\
\hline \multicolumn{11}{|l|}{ Real effective exchange rate change) } \\
\hline (annual average, percent change) & -10.7 & -6.8 & -4.1 & 2.2 & $\cdots$ & $\cdots$ & $\cdots$ & $\ldots$ & $\ldots$ & $\ldots$ \\
\hline Stock market index & 457 & 637 & 1,309 & 1,184 & $\ldots$ & $\ldots$ & $\ldots$ & $\ldots$ & $\ldots$ & $\ldots$ \\
\hline
\end{tabular}

Sources: Lebanese authorities; and Fund staff estimates.

$1 /$ Defined as cash in circulation plus resident and non-resident deposits.

$2 /$ Short-term debt on a remaining maturity basis. 
Table 2. Lebanon: Central Government Primary Balance, 2003-08

(In billions of Lebanese pounds)

\begin{tabular}{|c|c|c|c|c|c|c|c|c|c|c|}
\hline & 2003 & & 2005 & 2006 & & & 2007 & & & \\
\hline & $\begin{array}{l}\text { Year } \\
\text { Act. }\end{array}$ & $\begin{array}{l}\text { Year } \\
\text { Act. }\end{array}$ & $\begin{array}{l}\text { Year } \\
\text { Act. ?r }\end{array}$ & $\begin{array}{l}\text { Year } \\
\text { el. Act. }\end{array}$ & $\begin{array}{l}\text { Q1 } \\
\text { Prog. }\end{array}$ & $\begin{array}{l}\text { Q2 } \\
\text { Prog. }\end{array}$ & $\begin{array}{l}\text { Q3 } \\
\text { Prog. }\end{array}$ & $\begin{array}{l}\text { Q4 } \\
\text { Prog. }\end{array}$ & $\begin{array}{l}\text { Year } \\
\text { Prog. }\end{array}$ & $\begin{array}{l}\text { Year } \\
\text { Proj. }\end{array}$ \\
\hline Primary balance & 975 & 1,128 & 680 & 578 & -575 & 45 & 508 & 22 & -1 & 41 \\
\hline Revenue and grants & 6,597 & 7,484 & 7,405 & 8,486 & 1,763 & 2,360 & 2,846 & 2,565 & 9,534 & 9,62 \\
\hline Revenue & 6,597 & 7,484 & 7,405 & 7,490 & 1,763 & 2,042 & 2,181 & 2,231 & 8,218 & 8,813 \\
\hline Tax revenue & 4,527 & 5,169 & 4,867 & 4,922 & 1,175 & 1,428 & 1,376 & 1,415 & 5,394 & 6,309 \\
\hline Taxes on income and profits & 783 & 908 & 1,047 & 1,166 & 281 & 522 & 230 & 202 & 1,236 & 1,316 \\
\hline Taxes on property & 321 & 405 & 414 & 579 & 106 & 86 & 118 & 132 & 441 & 522 \\
\hline Taxes on domestic goods and services & 1,560 & 1,971 & 1,896 & 1,844 & 430 & 438 & 533 & 595 & 1,996 & 2,617 \\
\hline of which: VAT revenues & 1,386 & 1,763 & 1,693 & 1,659 & 389 & 396 & 480 & 537 & 1,802 & 2,387 \\
\hline Taxes on international trade $1 /$ & 1,645 & 1,617 & 1,268 & 1,074 & 300 & 314 & 414 & 422 & 1,450 & 1,567 \\
\hline Tariffs & 475 & 530 & 481 & 461 & 109 & 123 & 126 & 135 & 493 & 509 \\
\hline Excises & 1,170 & 1,087 & 787 & 613 & 191 & 191 & 287 & 287 & 957 & 1,058 \\
\hline Other taxes & 217 & 268 & 241 & 259 & 59 & 67 & 81 & 65 & 271 & 286 \\
\hline Nontax revenue & 2,070 & 2,316 & 2,538 & 2,568 & 588 & 614 & 806 & 816 & 2,824 & 2,504 \\
\hline Entrepreneurial and property income & 1,252 & 1,419 & 1,663 & 1,702 & 393 & 389 & 568 & 564 & 1,914 & 1,408 \\
\hline Profit transfer from BdL & 0 & 0 & 0 & 0 & 0 & 38 & 38 & 38 & 113 & \\
\hline Other & 1,252 & 1,419 & 1,662 & 1,702 & 393 & 352 & 530 & 526 & 1,801 & 1,408 \\
\hline Administrative fees and charges & 383 & 365 & 365 & 426 & 90 & 93 & 99 & 111 & 393 & 41 \\
\hline Other nontax revenue & 81 & 93 & 89 & 91 & 23 & 24 & 23 & 22 & 92 & 231 \\
\hline Fines and forfeits & 6 & 5 & 4 & 4 & 1 & 1 & 2 & 1 & 4 & \\
\hline Other & 75 & 88 & 85 & 87 & 22 & 23 & 22 & 22 & 88 & 227 \\
\hline Other treasury revenue & 354 & 439 & 421 & 349 & 82 & 108 & 115 & 119 & 425 & 450 \\
\hline Grants & 0 & 0 & 0 & 996 & 0 & 318 & 665 & 333 & 1,316 & 813 \\
\hline Primary expenditure $2 /$ & 5,622 & 6,356 & 6,725 & 7,908 & 2,338 & 2,315 & 2,338 & 2,543 & 9,535 & 9,213 \\
\hline Current primary expenditure & 4,708 & 5,304 & 6,025 & 7,051 & 2,008 & 1,901 & 1,827 & 2,034 & 7,770 & 8,093 \\
\hline Wages, salaries and pensions & 3,078 & 3,094 & 3,193 & 3,307 & 856 & 901 & 834 & 938 & 3,529 & 3,605 \\
\hline Wages and salaries & 2,234 & 2,284 & 2,329 & 2,386 & 643 & 652 & 592 & 681 & 2,568 & 2,561 \\
\hline Pensions & 844 & 810 & 864 & 927 & 213 & 250 & 241 & 257 & 961 & 1,044 \\
\hline Transfers to EDL 3 / & 174 & 184 & 637 & 1,137 & 315 & 338 & 339 & 458 & 1,450 & 1,615 \\
\hline Other current & 1,456 & 2,026 & 2,195 & 2,607 & 837 & 662 & 655 & 637 & 2,790 & 2,872 \\
\hline Materials and supplies & 120 & 116 & 213 & 140 & 68 & 55 & 34 & 38 & 195 & 200 \\
\hline External services & 81 & 113 & 82 & 87 & 21 & 29 & 17 & 23 & 90 & 92 \\
\hline Transfers 4/ 5/ & 271 & 360 & 655 & 878 & 419 & 166 & 104 & 131 & 819 & 850 \\
\hline o/w NSSF & $\ldots$ & 89 & 290 & 200 & 230 & 0 & 0 & 0 & 230 & 182 \\
\hline Other & 440 & 452 & 377 & 507 & 176 & 171 & 239 & 228 & 814 & 836 \\
\hline o/w "Housing compensations" 6 / & $\cdots$ & $\cdots$ & $\ldots$ & 136 & 60 & 100 & 160 & 132 & 452 & 465 \\
\hline Other treasury outflows $7 /$ & 544 & 985 & 868 & 995 & 153 & 241 & 260 & 218 & 872 & 894 \\
\hline Capital expenditure & 914 & 1,052 & 700 & 857 & 331 & 414 & 511 & 509 & 1,765 & 1,120 \\
\hline Domestically financed & 713 & 817 & 534 & 446 & 116 & 139 & 86 & 149 & 490 & 579 \\
\hline Foreign financed & 201 & 235 & 166 & 411 & 215 & 275 & 425 & 360 & 1,275 & 541 \\
\hline o/w conflict reconstruction 6 / & $\cdots$ & $\cdots$ & $\cdots$ & 111 & 120 & 180 & 330 & 265 & 895 & 95 \\
\hline \multicolumn{11}{|l|}{ Memorandum items: } \\
\hline Underlying primary balance (excl. conflict impact) & 975 & 1,128 & 680 & -73 & -395 & 7 & 333 & 85 & 30 & 161 \\
\hline Total conflict-related budgetary spending & $\ldots$ & $\ldots$ & $\ldots$ & 345 & 180 & 280 & 490 & 397 & 1,347 & 560 \\
\hline Primary balance excluding grants & 975 & 1,128 & 680 & -418 & -575 & -273 & -157 & -312 & $-1,317$ & -400 \\
\hline
\end{tabular}

Sources: Ministry of Finance; and Fund staff estimates and projections.

$1 /$ Domestic excises, which are collected at customs, are classified as taxes on international trade.

2/ On checks issued basis.

3/ Excludes principal and interest payments paid on behalf of EdL.

4/ From 2005 onward includes additional transfers to the social security funds (NSSF) to clear the stock of arrears.

$5 /$ Includes (i) from 2006 to 2008 transfers for telecom settlements of $\$ 97$ million, $\$ 30$ million, and $\$ 150$ million; and (ii) transfers to the Council of the South and the Displaced Fund (LL 750 billion split between 2007 to 2009).

$6 /$ The budgetary cost of the 2006 conflict is estimated to at $\$ 1.48$ billion.

$7 /$ includes transfers to municipalities. 
Table 3. Lebanon: Central Government Primary Balance, 2003-08

(in percent of GDP)

\begin{tabular}{|c|c|c|c|c|c|c|c|c|c|c|}
\hline & 2003 & 2004 & 2005 & 2006 & \multicolumn{5}{|c|}{2007} & \multirow{2}{*}{$\begin{array}{l}2008 \\
\text { Year } \\
\text { Proj. }\end{array}$} \\
\hline & $\begin{array}{l}\text { Year } \\
\text { Act. }\end{array}$ & $\begin{array}{l}\text { Year } \\
\text { Act. }\end{array}$ & $\begin{array}{l}\text { Year } \\
\text { Act. Pre }\end{array}$ & $\begin{array}{l}\text { Year } \\
\text { 1. Act. }\end{array}$ & $\begin{array}{l}\text { Q1 } \\
\text { Prog. }\end{array}$ & $\begin{array}{l}\text { Q2 } \\
\text { Prog. }\end{array}$ & $\begin{array}{l}\text { Q3 } \\
\text { Prog. }\end{array}$ & $\begin{array}{l}\text { Q4 } \\
\text { Prog. }\end{array}$ & $\begin{array}{l}\text { Year } \\
\text { Prog. }\end{array}$ & \\
\hline Primary balance & 3.3 & 3.5 & 2.1 & 1.7 & -1.6 & 0.1 & 1.4 & 0.1 & 0.0 & 1.1 \\
\hline Revenue and grants & 22.1 & 23.2 & 22.9 & 24.9 & 4.9 & 6.6 & 8.0 & 7.2 & 26.7 & 25.6 \\
\hline Revenue & 22.1 & 23.2 & 22.9 & 22.0 & 4.9 & 5.7 & 6.1 & 6.3 & 23.1 & 23.4 \\
\hline Tax revenue & 15.2 & 16.0 & 15.1 & 14.4 & 3.3 & 4.0 & 3.9 & 4.0 & 15.1 & 16.8 \\
\hline Taxes on income and profits & 2.6 & 2.8 & 3.2 & 3.4 & 0.8 & 1.5 & 0.6 & 0.6 & 3.5 & 3.5 \\
\hline Taxes on property & 1.1 & 1.3 & 1.3 & 1.7 & 0.3 & 0.2 & 0.3 & 0.4 & 1.2 & 1.4 \\
\hline Taxes on domestic goods and services & 5.2 & 6.1 & 5.9 & 5.4 & 1.2 & 1.2 & 1.5 & 1.7 & 5.6 & 7.0 \\
\hline of which: VAT revenues & 4.6 & 5.5 & 5.2 & 4.9 & 1.1 & 1.1 & 1.3 & 1.5 & 5.1 & 6.3 \\
\hline Taxes on international trade $1 /$ & 5.5 & 5.0 & 3.9 & 3.1 & 0.8 & 0.9 & 1.2 & 1.2 & 4.1 & 4.2 \\
\hline Tariffs & 1.6 & 1.6 & 1.5 & 1.4 & 0.3 & 0.3 & 0.4 & 0.4 & 1.4 & 1.4 \\
\hline Excises & 3.9 & 3.4 & 2.4 & 1.8 & 0.5 & 0.5 & 0.8 & 0.8 & 2.7 & 2.8 \\
\hline Other taxes & 0.7 & 0.8 & 0.7 & 0.8 & 0.2 & 0.2 & 0.2 & 0.2 & 0.8 & 0.8 \\
\hline Nontax revenue & 6.9 & 7.2 & 7.9 & 7.5 & 1.6 & 1.7 & 2.3 & 2.3 & 7.9 & 6.7 \\
\hline Entrepreneurial and property income & 4.2 & 4.4 & 5.1 & 5.0 & 1.1 & 1.1 & 1.6 & 1.6 & 5.4 & 3.7 \\
\hline Administrative fees and charges & 1.3 & 1.1 & 1.1 & 1.2 & 0.3 & 0.3 & 0.3 & 0.3 & 1.1 & 1.1 \\
\hline Other nontax revenue & 0.3 & 0.3 & 0.3 & 0.3 & 0.1 & 0.1 & 0.1 & 0.1 & 0.3 & 0.6 \\
\hline Other treasury revenue & 1.2 & 1.4 & 1.3 & 1.0 & 0.2 & 0.3 & 0.3 & 0.3 & 1.2 & 1.2 \\
\hline Grants & 0.0 & 0.0 & 0.0 & 2.9 & 0.0 & 0.9 & 1.9 & 0.9 & 3.7 & 2.2 \\
\hline Primary expenditure 2/ & 18.8 & 19.7 & 20.8 & 23.2 & 6.6 & 6.5 & 6.6 & 7.1 & 26.7 & 24.5 \\
\hline Current primary expenditure & 15.8 & 16.5 & 18.7 & 20.7 & 5.6 & 5.3 & 5.1 & 5.7 & 21.8 & 21.5 \\
\hline Wages, salaries and pensions & 10.3 & 9.6 & 9.9 & 9.7 & 2.4 & 2.5 & 2.3 & 2.6 & 9.9 & 9.6 \\
\hline Wages and salaries & 7.5 & 7.1 & 7.2 & 7.0 & 1.8 & 1.8 & 1.7 & 1.9 & 7.2 & 6.8 \\
\hline Pensions & 2.8 & 2.5 & 2.7 & 2.7 & 0.6 & 0.7 & 0.7 & 0.7 & 2.7 & 2.8 \\
\hline Transfers to EDL $3 /$ & 0.6 & 0.6 & 2.0 & 3.3 & 0.9 & 0.9 & 1.0 & 1.3 & 4.1 & 4.3 \\
\hline Other current & 4.9 & 6.3 & 6.8 & 7.6 & 2.3 & 1.9 & 1.8 & 1,8 & 7.8 & 7.6 \\
\hline Materials and supplies & 0.4 & 0.4 & 0.7 & $0.4^{*}$ & 0.2 & 0.2 & 0.1 & 0.1 & 0.5 & 0.5 \\
\hline External services & 0.3 & 0.4 & 0.3 & 0.3 & 0.1 & 0.1 & 0.0 & 0.1 & 0.3 & 0.2 \\
\hline Transfers 4/ 5/ & 0.9 & 1.1 & 2.0 & 2.6 & 1.2 & 0.5 & 0.3 & 0.4 & 2.3 & 2.3 \\
\hline o/w NSSF & $\ldots$ & 0.3 & 0.9 & 0.6 & 0.6 & 0.0 & 0.0 & 0.0 & 0.6 & 0.5 \\
\hline Other & 1.5 & 1.4 & 1.2 & 1.5 & 0.5 & 0.5 & 0.7 & 0.6 & 2.3 & 2.2 \\
\hline o/w "Housing compensations" $6 /$ & $\ldots$ & $\ldots$ & $\ldots$ & 0.4 & 0.2 & 0.3 & 0.4 & 0.4 & 1.3 & 1.2 \\
\hline Other treasury outfiows $7 /$ & 1.8 & 3.1 & 2.7 & 2.9 & 0.4 & 0.7 & 0.7 & 0.6 & 2.4 & 2.4 \\
\hline Capital expenditure & 3.1 & 3.3 & 2.2 & 2.5 & 0.9 & 1.2 & 1.4 & 1.4 & 5.0 & 3.0 \\
\hline Domestically financed & 2.4 & 2.5 & 1.7 & 1.3 & 0.3 & 0.4 & 0.2 & 0.4 & 1.4 & 1.5 \\
\hline Foreign financed & 0.7 & 0.7 & 0.5 & 1.2 & 0.6 & 0.8 & 1.2 & 1.0 & 3.6 & 1.4 \\
\hline o/w conflict reconstruction 6 / & $\cdots$ & $\cdots$ & $\cdots$ & 0.3 & 0.3 & 0.5 & 0.9 & 0.7 & 2.5 & 0.3 \\
\hline \multicolumn{11}{|l|}{ Memorandum items: } \\
\hline Underlying primary balance (excl. conflict impact) & 3.3 & 3.5 & 2.1 & -0.2 & -1.1 & 0.0 & 0.9 & 0.2 & 0.1 & 0.4 \\
\hline Total conflict-related budgetary spending & $\ldots$ & $\ldots$ & $\ldots$ & 1.0 & 0.5 & 0.8 & .1 .4 & 1.1 & 3.8 & 1.5 \\
\hline Primary balance excluding grants & 3.3 & 3.5 & 2.1 & -1.2 & -1.6 & -0.8 & -0.4 & -0.9 & -3.7 & -1.1 \\
\hline
\end{tabular}

Sources: Ministry of Finance; and Fund staff estimates and projections.

$1 /$ Domestic excises, which are collected at customs, are classified as taxes on international trade.

$2 /$ On checks issued basis.

3/ Excludes principal and interest payments paid on behalf of EdL.

4/ From 2005 onward includes additional transfers to the social security funds (NSSF) to clear the stock of arrears.

5/ Includes (i) from 2006 to 2008 transfers for telecom settlements of $\$ 97$ million, $\$ 30$ million, and $\$ 150$ million; and (ii) transfers to the Council of the South and the Displaced Fund (LL 750 billion split between 2007 to 2009).

6/ The budgetary cost of the 2006 conflict is estimated to at $\$ 1.48$ billion.

$7 /$ Includes transfers to municipalities. 
Table 4. Lebanon: Overall Fiscal Deficit and Financing, 2003-08

\begin{tabular}{|c|c|c|c|c|c|c|c|c|c|c|}
\hline & 2003 & 2004 & 2005 & 2006 & \multicolumn{5}{|c|}{2007} & \multirow{2}{*}{$\begin{array}{l}2008 \\
\text { Year } \\
\text { Proj. }\end{array}$} \\
\hline & $\begin{array}{l}\text { Year } \\
\text { Act. }\end{array}$ & $\begin{array}{l}\text { Year } \\
\text { Act. }\end{array}$ & $\begin{array}{l}\text { Year } \\
\text { Act. }\end{array}$ & $\begin{array}{l}\text { Year } \\
\text { Act. }\end{array}$ & $\begin{array}{c}\text { Q1 } \\
\text { Proj. }\end{array}$ & $\begin{array}{c}\text { Q2 } \\
\text { Proj. }\end{array}$ & $\begin{array}{c}\text { Q3 } \\
\text { Proj. }\end{array}$ & $\begin{array}{c}\text { Q4 } \\
\text { Proj. }\end{array}$ & $\begin{array}{l}\text { Year } \\
\text { Proj. }\end{array}$ & \\
\hline & \multicolumn{10}{|c|}{ (In billions of Lebanese pounds) } \\
\hline Primary balance & 975 & 1,128 & 680 & 578 & -575 & 45 & 508 & 22 & -1 & 413 \\
\hline Interest bill & 4,942 & 3,921 & 3,410 & 4,381 & 1,071 & 1,070 & 1,248 & 1,018 & 4,408 & 4,417 \\
\hline Overall balance (checks-issued basis) & $-3,968$ & $-2,793$ & -2.730 & $-3,803$ & $-1,646$ & $-1,026$ & -740 & -997 & $-4,409$ & $-4,004$ \\
\hline Float and statistical discrepancy & 125 & 325 & 78 & -29 & $\ldots$ & $\ldots$ & $\ldots$ & $\ldots$ & $\ldots$ & $\ldots$ \\
\hline Overall balance (cash basis) & $-4,093$ & $-3,119$ & $-2,808$ & $-3,774$ & $-1,646$ & $-1,026$ & -740 & -997 & $-4,409$ & $-4,004$ \\
\hline Net financing & 4,093 & 3,119 & 2,808 & 3,774 & 1,646 & 1,026 & 740 & 997 & 4,409 & 4,004 \\
\hline Banking system & 3,555 & 4,269 & 3,188 & 1,904 & 1,481 & $-1,943$ & 243 & -573 & -793 & $-8,287$ \\
\hline Banque du Liban 1/ & 8,779 & 655 & 601 & $-2,342$ & 905 & $-1,460$ & -540 & $-1,066$ & $-2,162$ & $-5,388$ \\
\hline Commercial banks 1 / & $-5,224$ & 3,614 & 2.587 & 4,247 & 576 & -484 & 783 & 493 & 1,369 & $-2,900$ \\
\hline Government institutions & -605 & -352 & 245 & 835 & 61 & 500 & -120 & -93 & 348 & 367 \\
\hline Other creditors & $-1,375$ & $-1,184$ & -472 & 1.186 & 105 & -212 & -295 & 188 & -216 & -761 \\
\hline Net change in arrears & 0 & 0 & -419 & 0 & 0 & 0 & 0 & 0 & 0 & 0 \\
\hline Exceptional financing & 2,999 & 600 & -132 & 39 & 0 & 2,682 & 913 & 1,475 & 5,070 & 12,686 \\
\hline Privatization & -280 & 0 & 0 & 0 & 0 & 0 & 0 & 0 & 0 & 11,743 \\
\hline Bilateral and multilateral & 3,279 & 83 & -132 & 39 & 0 & 302 & 913 & 1,475 & 2,690 & 942 \\
\hline Other $2 /$ & 0 & 517 & 0 & 0 & 0 & 2,380 & 0 & 0 & 2,380 & 0 \\
\hline \multirow[t]{2}{*}{ Valuation adjustment } & -480 & -215 & 399 & -190 & 0 & 0 & 0 & 0 & 0 & 0 \\
\hline & \multicolumn{10}{|c|}{ (In percent of GDP) } \\
\hline Primary balance & 3.3 & 3.5 & 2.1 & 1.7 & -1.6 & 0.1 & 1.4 & 0.1 & 0.0 & 1.1 \\
\hline Interest bill & 16.6 & 12.2 & 10.6 & 12.8 & 3.0 & 3.0 & 3.5 & 2.9 & 12.4 & 11.7 \\
\hline Overall balance (checks-issued basis) & -13.3 & -8.7 & -8.5 & -11.2 & -4.6 & -2.9 & -2.1 & -2.8 & -12.4 & -10.6 \\
\hline Float and statistical discrepancy & 0.4 & 1.0 & 0.2 & -0.1 & $\ldots$ & $\ldots$ & $\ldots$ & ... & $\ldots$ & $\ldots$ \\
\hline Overall balance (cash basis) & -13.7 & -9.7 & -8.7 & -11.1 & -4.6 & -2.9 & -2.1 & -2.8 & -12.4 & -10.6 \\
\hline Net financing & 13.7 & 9.7 & 8.7 & 11.1 & 4.6 & 2.9 & 2.1 & 2.8 & 12.4 & 10.6 \\
\hline Banking system & 11.9 & 13.3 & 9.9 & 5.6 & 4.2 & -5.5 & 0.7 & -1.6 & .2 .2 & -22.0 \\
\hline Banque du Liban 1/ & 29.4 & 2.0 & 1.9 & -6.9 & 2.5 & -4.1 & -1.5 & -3.0 & -6.1 & -14.3 \\
\hline Commercial banks $1 /$ & -17.5 & 11.2 & 8.0 & 12.5 & 1.6 & -1.4 & 2.2 & 1.4 & 3.8 & -7.7 \\
\hline Government institutions & -2.0 & -1.1 & 0.8 & 2.4 & 0.2 & 1.4 & -0.3 & -0.3 & 1.0 & 1.0 \\
\hline Other creditors & -4.6 & -3.7 & -1.5 & 3.5 & 0.3 & -0.6 & -0.8 & 0.5 & -0.6 & -2.0 \\
\hline Net change in arrears & 0.0 & 0.0 & -1.3 & 0.0 & 0.0 & 0.0 & 0.0 & 0.0 & 0.0 & 0.0 \\
\hline Exceptional financing & 10.0 & 1.9 & -0.4 & 0.1 & 0.0 & 7.5 & 2.6 & 4.1 & 14.2 & 33.7 \\
\hline Privatization & -0.9 & 0.0 & 0.0 & 0.0 & 0.0 & 0.0 & 0.0 & 0.0 & 0.0 & 31.2 \\
\hline Bilateral and multilateral & 11.0 & 0.3 & -0.4 & 0.1 & 0.0 & 0.8 & 2.6 & 4.1 & 7.5 & 2.5 \\
\hline Other $2 /$ & 0.0 & 1.6 & 0.0 & 0.0 & 0.0 & 6.7 & 0.0 & 0.0 & 67 & 0.0 \\
\hline Valuation adjustment & -1.6 & -0.7 & 1.2 & -0.6 & 0.0 & 0.0 & 0.0 & 0.0 & 0.0 & 0.0 \\
\hline \multicolumn{11}{|l|}{ Memorandum item: } \\
\hline Nominal GDP (annual) & 29,851 & 32,214 & 32,303 & 34,102 & 35,647 & 35,647 & 35,647 & 35,647 & 35,647 & 37,633 \\
\hline
\end{tabular}

Sources: Lebanese authorities; and Fund staff estimates and calculations.

1/ Figures in 2003 are affected by the role played by the Banque du Liban (BdL) in the debt exchange with banks that tends to increase BdL financing and decrease commercial bank financing of the government.

2/ Debt cancellation and Banque du Liban revaluation of gold and foreign exchange. 


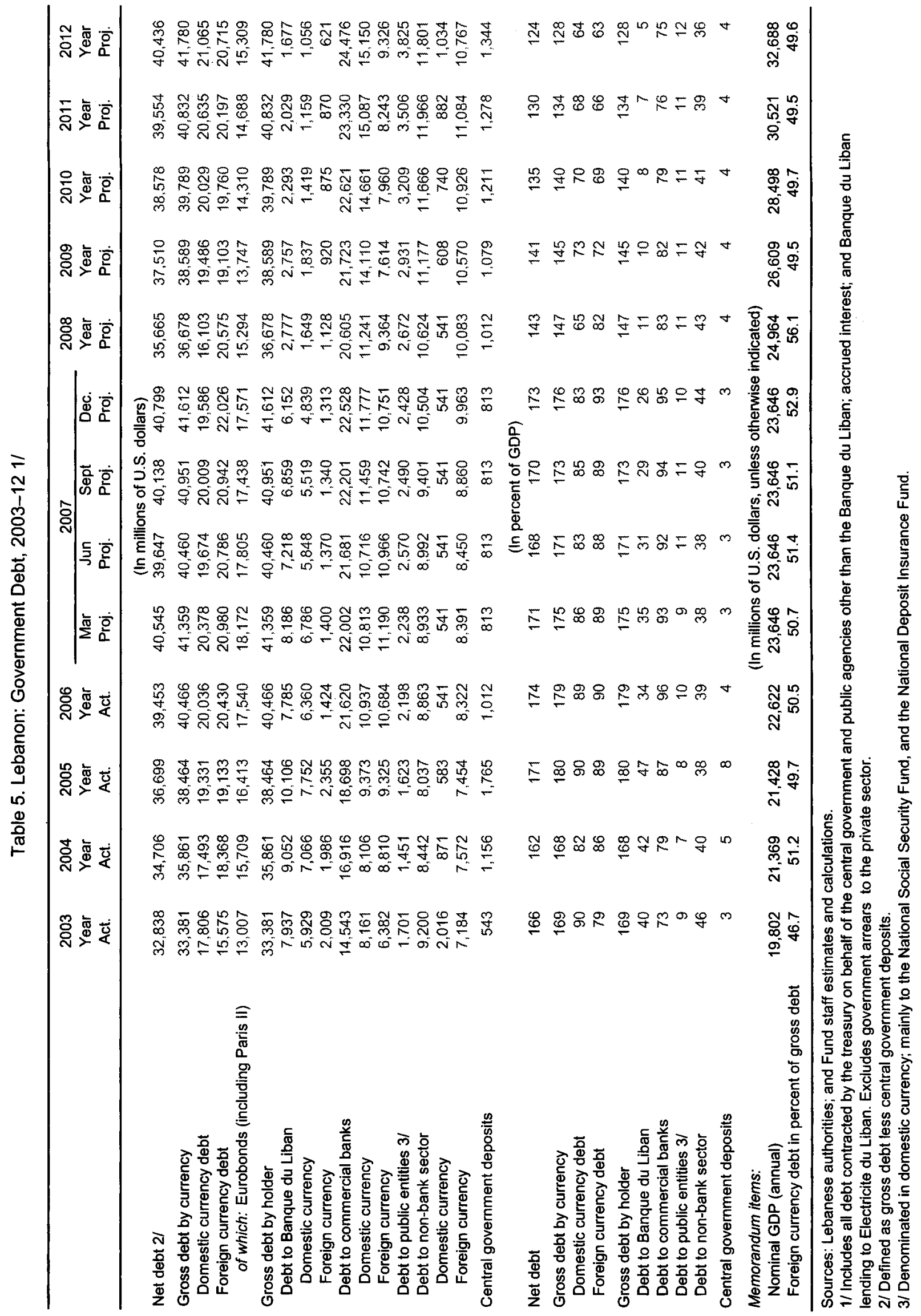


Table 6. Lebanon: Monetary Survey, 2003-08

\begin{tabular}{|c|c|c|c|c|c|c|c|c|c|}
\hline & 2003 & 2004 & 2005 & 2006 & \multicolumn{4}{|c|}{2007} & \multirow{3}{*}{$\begin{array}{l}2008 \\
\text { Year } \\
\text { Proj. }\end{array}$} \\
\hline & Year & Year & Year & Year & Mar. & Jun. & Sep. & Dec. & \\
\hline & & & & & Proj. & Proj. & Proj. & Proj. & \\
\hline & \multicolumn{9}{|c|}{ (Stocks in billions of Lebanese pounds) } \\
\hline Net foreign assets & 33,509 & 36,803 & 37,832 & 43,594 & 41,460 & 41,379 & 41,418 & 43,181 & 49,776 \\
\hline Banque du Liban & 20,970 & 20,185 & 21,078 & 23,047 & 22,211 & 21,630 & 21,169 & 22,132 & 26,227 \\
\hline Commercial banks & 12,539 & 16,618 & 16,754 & 20,547 & 19,249 & 19,749 & 20,249 & 21,049 & 23,549 \\
\hline Net domestic assets & 42,848 & 48,930 & 50,888 & 50,784 & 52,446 & 53,466 & 54,376 & 54,529 & 54,773 \\
\hline Net claims on public sector & 30,670 & 34,029 & 36,891 & 38,619 & 40,100 & 38,156 & 38,399 & 37,826 & 29,539 \\
\hline of which: Net claims on government & 31,989 & 35,646 & 38,898 & 40,984 & 42,465 & 40,522 & 40,765 & 40,192 & 31,904 \\
\hline Banque du Liban & 10,983 & 11,491 & 12,201 & 9,791 & 10,696 & 9,237 & 8,696 & 7,630 & 2,242 \\
\hline Lebanese pounds & 8,278 & 9,415 & 9,537 & 8,935 & 9,576 & 8,161 & 7,665 & 6,640 & 1,773 \\
\hline Foreign currency & 2,706 & 2,076 & 2,664 & 856 & 1,121 & 1,076 & 1,031 & 990 & 469 \\
\hline Commercial banks & 21,006 & 24,155 & 26,697 & 31,193 & 31,769 & 31,285 & 32,068 & 32,562 & 29,662 \\
\hline Lebanese pounds & 11,366 & 10,853 & 12,608 & 15,043 & 14,858 & 14,713 & 15,835 & 16,317 & 15,515 \\
\hline Foreign currency & 9,640 & 13,302 & 14,089 & 16,150 & 16,911 & 16,572 & 16,234 & 16,245 & 14,147 \\
\hline Claims on private sector & 23,233 & 24,375 & 24,774 & 26,209 & 26,345 & 26,657 & 27,053 & 27,508 & 35,013 \\
\hline Lebanese pounds & 4,254 & 4,622 & 4,613 & 5,048 & 4,982 & 5,059 & 5,139 & 5,230 & 5,527 \\
\hline Foreign currency & 18,980 & 19,752 & 20,161 & 21,162 & 21,362 & 21,598 & 21,914 & 22,278 & 29,486 \\
\hline Other items (net) & $-11,056$ & $-9,474$ & $-10,777$ & $-14,044$ & $-13,998$ & $-11,347$ & $-11,076$ & $-10,805$ & $-9,778$ \\
\hline Broad money (M5) 1/ & 76,357 & 85,733 & 88,720 & 94,378 & 93,906 & 94,845 & 95,794 & 97,710 & 104,549 \\
\hline In Lebanese pounds & 27,458 & 27,427 & 25,503 & 24,159 & 24,138 & 24,379 & 24,620 & 25,580 & 27,863 \\
\hline Currency in circulation & 1,531 & 1,586 & 1,535 & 1,809 & 1,667 & 1,685 & 1,706 & 1,742 & 1,864 \\
\hline Deposits in Lebanese pounds & 25,927 & 25,840 & 23,968 & 22,350 & 22,471 & 22,695 & 22,914 & 23,838 & 26,000 \\
\hline \multirow[t]{2}{*}{ Deposits in foreign currency } & 48,900 & 58,306 & 63,217 & 70,219 & 69,768 & 70,466 & 71,174 & 72,130 & 76,686 \\
\hline & & \multicolumn{8}{|c|}{ (Year-to-date change in billions of Lebanese pounds) } \\
\hline Net foreign assets & 8.754 & 3,294 & 1,029 & 5,762 & $-2,134$ & $-2,215$ & $-2,176$ & -413 & 6,595 \\
\hline Net domestic assets & 1,502 & 6,082 & 1,959 & -104 & 1,662 & 2,682 & 3,592 & 3,744 & 245 \\
\hline Net claims on public sector & 2,480 & 3,359 & 2,863 & 1,727 & 1,481 & -462 & -220 & -793 & $-8,287$ \\
\hline Net claims on government & 2,912 & 3,658 & 3,251 & 2,086 & 1,481 & -462 & -220 & -793 & $-8,287$ \\
\hline Banque du Liban & 8,484 & 508 & 710 & $-2,410$ & 905 & -555 & $-1,095$ & $-2,162$ & $-5,388$ \\
\hline Commercial banks & $-5,572$ & 3,150 & 2,541 & 4,496 & 576 & 92 & 876 & 1,369 & $-2,900$ \\
\hline Broad money (M5) 1/ & 10,256 & 9,375 & 2,987 & 5,658 & -472 & 467 & 1,416 & 3,332 & 6,840 \\
\hline In Lebanese pounds & 6,338 & -31 & $-1,924$ & $-1,344$ & -21 & 220 & 461 & 1,421 & 2,284 \\
\hline \multirow[t]{2}{*}{ Deposits in foreign currency } & 3,918 & 9,406 & 4,911 & 7,002 & -451 & 247 & 955 & 1,911 & 4,556 \\
\hline & \multicolumn{9}{|c|}{ (Year-to-date change in percent of beginning of period broad money) } \\
\hline Broad money (M5) 1/ & 15.5 & 12.3 & 3.5 & 6.4 & -0.5 & 0.5 & 1.5 & 3.5 & 7.0 \\
\hline In Lebanese pounds & 9.6 & 0.0 & -2.2 & -1.5 & 0.0 & 0.2 & 0.5 & 1.5 & 2.3 \\
\hline Deposits in foreign currency & 5.9 & 12.3 & 5.7 & 7.9 & -0.5 & 0.3 & 1.0 & 2.0 & 4.7 \\
\hline \multicolumn{10}{|l|}{ Memorandum items: } \\
\hline Net foreign assets (in millions of U.S. dollars) & 22,228 & 24,413 & 25,096 & 28,918 & 27,503 & 27,449 & 27,475 & 28,644 & 33,019 \\
\hline Share of foreign currency deposits in total private sector deposits & 66.2 & 70.1 & 73.2 & 76.2 & 76.0 & 76.0 & 76.0 & 75.5 & 75.0 \\
\hline Credit to private sector (in percent of GDP) & 77.8 & 75.7 & 76.7 & 76.9 & $\ldots$ & $\ldots$ & $\ldots$ & 77.2 & 93.0 \\
\hline Credit to private sector (in twelve month percent change) & 0.3 & 5.2 & 1.9 & 6.0 & 5.0 & 3.3 & 4.6 & 5.0 & 27.5 \\
\hline M5 to GDP (in percent) & 255.8 & 266.1 & 274.6 & 276.8 & $\ldots$ & $\ldots$ & $\ldots$ & 274.1 & 277.8 \\
\hline
\end{tabular}

Sources: Banque du Liban; and Fund staff estimates and projections.

$1 /$ Broad money is taken to be M5 which is defined as M3 (currency + resident deposits) + non-resident deposits. 
Table 7. Lebanon: Balance Sheet of the Banque du Liban, 2003-08

\begin{tabular}{|c|c|c|c|c|c|c|c|c|c|}
\hline & 2003 & 2004 & 2005 & 2006 & & 200 & & & 2008 \\
\hline & $\begin{array}{c}\text { Year } \\
\text { Act. }\end{array}$ & $\begin{array}{c}\text { Year } \\
\text { Act. }\end{array}$ & $\begin{array}{c}\text { Year } \\
\text { Act. }\end{array}$ & $\begin{array}{c}\text { Year } \\
\text { Act. }\end{array}$ & $\begin{array}{l}\text { Mar. } \\
\text { Proj. }\end{array}$ & $\begin{array}{l}\text { Jun. } \\
\text { Proj. }\end{array}$ & $\begin{array}{l}\text { Sep. } \\
\text { Proj. }\end{array}$ & $\begin{array}{l}\text { Dec. } \\
\text { Proi. }\end{array}$ & $\begin{array}{l}\text { Year } \\
\text { Proj. }\end{array}$ \\
\hline & \multicolumn{9}{|c|}{ (Stocks in billions of Lebanese pounds) } \\
\hline Net foreign exchange position $1 /$ & 8,935 & 6,712 & 2,873 & 3,465 & 2,449 & 1,259 & 1,521 & 2,684 & 7,653 \\
\hline Foreign assets & 21,263 & 20,473 & 22,081 & 26,321 & 25,485 & 25,019 & 24,607 & 25,570 & 29,639 \\
\hline of which: gold & 5,779 & 6,039 & 7,140 & 8,755 & 9,379 & 9,379 & 9,379 & 9,379 & 9,379 \\
\hline Foreign currency liabilities & 12,328 & 13,762 & 19,208 & 22,856 & 23,036 & 23,760 & 23,086 & 22,886 & 21,986 \\
\hline of which: commercial bank deposits $2 /$ & 11,776 & 13,213 & 17,901 & 19,189 & 19,382 & 19,990 & 19,268 & 19,067 & 18,194 \\
\hline of which: other foreign liabilities $3 /$ & 293 & 288 & 1,003 & 3,274 & 3,274 & 3,390 & 3,439 & 3,439 & 3,412 \\
\hline Net.domestic assets & $-2,528$ & 1,411 & 4,491 & 3,762 & 4,893 & 6,154 & 5,962 & 5,052 & 800 \\
\hline Claims on public sector (net) & 10,990 & 11,354 & 11,900 & 9,005 & 9,910 & 8,450 & 7,910 & 6,843 & 1,456 \\
\hline Net claims on government & 10,983 & 11,491 & 12,201 & 9,791 & 10,696 & 9,237 & 8,696 & 7,630 & 2,242 \\
\hline Credit & 11,800 & 13,232 & 14,860 & 11,318 & 11,922 & 10,463 & 9,922 & 8,856 & 3,768 \\
\hline Treasury billis & 8,772 & 10,238 & 11,310 & 9,170 & 9,813 & 8,398 & 7,902 & 6,877 & 2,068 \\
\hline Eurobonds & 3,028 & 2,994 & 3,550 & 2,147 & 2,110 & 2,065 & 2,020 & 1,979 & 1,700 \\
\hline Deposits & 817 & 1,741 & 2,659 & 1,526 & 1,226 & 1,226 & 1,226 & 1,226 & 1,526 \\
\hline Net claims on non financial public institutions & 7 & -138 & -302 & -787 & -787 & -787 & -787 & -787 & -787 \\
\hline Claims on private sector in Lebanese pounds (net) & 375 & 313 & 257 & 232 & 240 & 240 & 240 & 240 & 240 \\
\hline Claims on commercial banks & $-9,859$ & $-7,657$ & $-6,163$ & $-3,796$ & $-3,879$ & $-3,810$ & $-3,732$ & $-3,847$ & $-3,743$ \\
\hline of which: Certificates of deposit in Lebanese pounds & 11,686 & 9,516 & 7,959 & 5,639 & 5,639 & 5,570 & 5,492 & 5,608 & 5,503 \\
\hline Claims on specialized banks in Lebanese pounds (net) & $-1,032$ & $-1,004$ & -837 & -539 & -509 & -509 & -509 & -509 & -509 \\
\hline Other items (net) & $-3,002$ & $-1,595$ & -665 & $-1,140$ & -869 & 1,782 & 2,053 & 2,324 & 3,355 \\
\hline Reserve money & 6,407 & 8,123 & 7,364 & 7,227 & 7,342 & 7,413 & 7,483 & 7,736 & 8,453 \\
\hline Currency issued & 1,717 & 1,783 & 1,736 & 2,010 & 1,916 & 1,935 & 1,954 & 1,993 & 2,133 \\
\hline \multirow[t]{2}{*}{ Commercial bank deposits in Lebanese pounds } & 4,690 & 6,340 & 5,628 & 5,217 & 5,426 & 5,478 & 5,529 & 5,743 & 6,320 \\
\hline & \multicolumn{9}{|c|}{ (Year-to-date flows in billions of Lebanese pounds) } \\
\hline Reserve money & 1,753 & 1,715 & -759 & -137 & 115 & 186 & 256 & 509 & 717 \\
\hline Currency issued & 176 & 66 & -47 & 274 & -95 & -75 & -56 & -17 & 140 \\
\hline \multirow[t]{2}{*}{ Commercial bank deposits in Lebanese pounds } & 1,577 & 1,649 & -711 & -412 & 210 & 262 & 312 & 526 & 577 \\
\hline & \multicolumn{9}{|c|}{ (Year-to-date changes in percent of beginning-of-period reserve money) } \\
\hline \multirow[t]{2}{*}{ Reserve money } & 38 & 27 & -9 & -2 & 2 & 3 & 4 & 7 & 9 \\
\hline & \multicolumn{9}{|c|}{ (In millions of U.S. dollars) } \\
\hline \multicolumn{10}{|l|}{ Memorandum items: } \\
\hline Gross international reserves (including gold) $4 /$ & 14,105 & 13,581 & 14,347 & $\uparrow 7,160$ & 16,605 & 16,296 & 16,023 & 16,662 & 19,361 \\
\hline Gross international reserves (excluding gold) $4 /$ & 10,271 & 9,575 & 9,611 & 11,353 & 10,383 & 10,075 & 9,801 & 10,440 & 13,139 \\
\hline in percent of banking system foreign currency deposits & 40 & 32 & 29 & 30 & 27 & 26 & 25 & 27 & 31 \\
\hline in percent of total banking system deposits & 25 & 21 & 20 & 22 & 20 & 19 & 18 & 19 & 22 \\
\hline Program gross reserves $5 /$ & 16,113 & 15,567 & 16,202 & 18,084 & 17,505 & 17,166 & 16,863 & 17,475 & 20,039 \\
\hline Lebanese pound money multiplier & 4.3 & 3.4 & 3.5 & 3.4 & 3.3 & 3.3 & 3.3 & 3.3 & 3.3 \\
\hline
\end{tabular}

Sources: Banque du Liban; and Fund staff estimates and projections.

1/ Defined by currency (not by residency), as official foreign currency assets, including gold and SDR, less foreign currency liabilities.

These include the $\$ 1.5$ billion deposits by the Saudi and Kuwaiti governments in August 2006, but exclude liabilities to the government of Lebanon and other official creditors.

$2 /$ Inctudes certificates of deposits in foreign currency held by commercial banks.

$3 /$ Includes the deposits by the Saudi and Kuwaiti governments. Excludes all other special bilateral long-term deposits.

4/ Defined as all official foreign currency assets, less encumbered foreign assets.

5/ Defined as gross international reserves including gold and Eurobonds issued by the Republic of Lebanon. 
Table 8. Lebanon: Commercial Banks' Balance Sheet, 2003-08

\begin{tabular}{|c|c|c|c|c|c|c|c|c|c|}
\hline & 2003 & & 2005 & 2006 & \multicolumn{4}{|c|}{2007} & \multirow{2}{*}{$\begin{array}{l}2008 \\
\text { Year } \\
\text { Proj. }\end{array}$} \\
\hline & $\begin{array}{c}\text { Year } \\
\text { Act. }\end{array}$ & $\begin{array}{c}\text { Year } \\
\text { Act. }\end{array}$ & $\begin{array}{c}\text { Year } \\
\text { Act. }\end{array}$ & $\begin{array}{c}\text { Year } \\
\text { Act. }\end{array}$ & $\begin{array}{l}\text { Mar. } \\
\text { Proj. }\end{array}$ & $\begin{array}{l}\text { Jun. } \\
\text { Proj. }\end{array}$ & $\begin{array}{l}\text { Sep. } \\
\text { Proj. }\end{array}$ & $\begin{array}{l}\text { Dec. } \\
\text { Proj. }\end{array}$ & \\
\hline & \multicolumn{9}{|c|}{ (Stocks in billions of Lebanese pounds) } \\
\hline Net foreign assets & 12,539 & 16,618 & 16,754 & 20,547 & 19,249 & 19,749 & 20,249 & 21,049 & 23,549 \\
\hline Foreign assets & 14,937 & 20,431 & 20,017 & 24,784 & 22,784 & 23,284 & 23,784 & 24,584 & 27,084 \\
\hline Foreign liabilities & 2,398 & 3,813 & 3,263 & 4,236 & 3,534 & 3,534 & 3,534 & 3,534 & 3,534 \\
\hline Net domestic assets & 60,974 & 66,223 & 69,240 & 71,042 & 72,047 & 72,468 & 72,896 & 73,975 & 78,198 \\
\hline Net claims on public sector & 19,680 & 22,675 & 24,992 & 29,614 & 30,190 & 29,706 & 30,489 & 30,983 & 28,083 \\
\hline of which: Treasury bills & 11,366 & 10,853 & 12,608 & 15,043 & 14,858 & 14,713 & 15,835 & 16,317 & 15.515 \\
\hline of which: Foreign currency bonds & 9,621 & 13,281 & 14,057 & 16,105 & 16,868 & 16,531 & 16,194 & 16,207 & 14,116 \\
\hline Claims on Banque du Liban & 28,339 & 29,265 & 31,690 & 30,245 & 30,696 & 31,289 & 30,537 & 30,669 & 30,286 \\
\hline Currency in vault & 186 & 197 & 201 & 273 & 248 & 250 & 248 & 251 & 269 \\
\hline Deposits with Banque du Liban & 16,466 & 19,552 & 23,530 & 24,405 & 24,808 & 25,469 & 24,796 & 24,810 & 24,514 \\
\hline Certificates of deposit in Lebanese pounds & 11,686 & 9,516 & 7,959 & 5,639 & 5,639 & 5,570 & 5,492 & 5,608 & 5,503 \\
\hline Claims on private sector & 22,836 & 24,020 & 24,467 & 25,930 & 26,059 & 26,372 & 26,768 & 27,223 & 34,728 \\
\hline Other items (net) & $-9,881$ & $-9,738$ & $-11,908$ & $-14,747$ & $-14,899$ & $-14,899$ & $=14,899$ & $-14,899$ & $-14,899$ \\
\hline Liabilities to private sector & 73,513 & 82,841 & 85,994 & 91,590 & 91,296 & 92,218 & 93,145 & 95,025 & 101,747 \\
\hline Lebanese pounds $1 /$ & 24,873 & 24,796 & 23,081 & 21,763 & 21,913 & 22,136 & 22,356 & 23,280 & 25,441 \\
\hline \multirow[t]{2}{*}{ Foreign currency $2 /$} & 48,641 & 58,046 & 62,914 & 69,826 & 69,383 & 70,081 & 70,790 & 71,745 & 76,306 \\
\hline & \multicolumn{9}{|c|}{ (Year-to-date flows in billions of Lebanese pounds) } \\
\hline Net foreign assets & 72 & 4,079 & 136 & 3,793 & $-1,298$ & -798 & -298 & 502 & 2,500 \\
\hline Net domestic assets & 9,010 & 5,249 & 3,017 & 1,802 & 1,004 & 1,426 & 1,853 & 2,933 & 4,222 \\
\hline Net claims on public sector & $-6,306$ & 2,995 & 2,316 & 4,622 & 576 & 92 & 876 & 1,369 & $-2,900$ \\
\hline Claims on Banque du Liban & 16,323 & 926 & 2,425 & $-1,444$ & 451 & 1,043 & 292 & 423 & -383 \\
\hline Claims on private sector & 78 & 1,185 & 446 & 1,463 & 130 & 442 & 838 & 1,293 & 7,505 \\
\hline Other items (net) & $-1,085$ & 143 & $-2,170$ & $-2,839$ & -152 & -152 & -152 & -152 & 0 \\
\hline Liabilities to private sector & 9,082 & 9,328 & 3,153 & 5,595 & -294 & 628 & 1,555 & 3,435 & 6,722 \\
\hline Lebanese pounds $1 /$ & 5,187 & -77 & $-1,715$ & $-1,318$ & 150 & 373 & 592 & 1,516 & 2,162 \\
\hline Foreign currency $2 /$ & 3,895 & 9,405 & 4,868 & 6,913 & -443 & 255 & 963 & 1,919 & 4,560 \\
\hline \multicolumn{10}{|l|}{ Memorandum items: } \\
\hline Ratio of foreign currency assets to liabilities & 1.1 & 1.1 & 1.1 & 1.1 & 1.10 & 1.11 & 1.09 & 1.09 & 1.10 \\
\hline Ratio of local currency assets to liabilities 3 / & 0.8 & 0.8 & 0.8 & 0.7 & 0.68 & 0.67 & 0.71 & 0.72 & 0.66 \\
\hline Exposure to the government (percent of total assets) & 23.2 & 23.6 & 25.2 & 27.2 & 27.8 & 27.1 & 27.5 & 27.3 & 23.3 \\
\hline Exposure to the sovereign (percent of total assets) & 54.5 & 52.3 & 55.1 & 53.5 & 54.6 & 54.1 & 53.6 & 53.1 & 47.0 \\
\hline
\end{tabular}

Source: Banque du Lỉban.

$1 /$ Includes nonresident deposits.

$2 /$ Includes bonds denominated in foreign currency.

3 / Includes other items net as assets. 
Table 9. Lebanon: Balance of Payments, 2003-12

\begin{tabular}{|c|c|c|c|c|c|c|c|c|c|c|}
\hline & $\begin{array}{l}2003 \\
\text { Act. }\end{array}$ & $\begin{array}{l}2004 \\
\text { Act. }\end{array}$ & $\begin{array}{l}2005 \\
\text { Act. }\end{array}$ & $\begin{array}{c}2006 \\
\text { Prel. Act. }\end{array}$ & $\begin{array}{l}2007 \\
\text { Proj. }\end{array}$ & $\begin{array}{l}2008 \\
\text { Proj. }\end{array}$ & $\begin{array}{l}2009 \\
\text { Proj. }\end{array}$ & $\begin{array}{l}2010 \\
\text { Proj- }\end{array}$ & $\begin{array}{l}2011 \\
\text { Proj. }\end{array}$ & $\begin{array}{l}2012 \\
\text { Proj. }\end{array}$ \\
\hline \multicolumn{11}{|c|}{ (in millions of U.S. dollars, unless otherwise indicated) } \\
\hline Current account & $-2,625$ & $-3,377$ & $-2,517$ & $-1,549$ & $-2,596$ & -2.495 & $-2,500$ & $-1,881$ & $-1,750$ & $-1,750$ \\
\hline Goods (net) & $-4,794$ & -6.452 & -6.118 & $-5,755$ & $-6,895$ & $-6,868$ & $-7,377$ & $-7,256$ & $-7,611$ & $-8,071$ \\
\hline Exports, f.o.b. & 1,733 & 2,050 & 2,278 & 2,792 & 3,151 & 3,613 & 4,120 & 4,738 & 5,425 & 6.165 \\
\hline Imports, f.o.b. & $-6,528$ & $-8,502$ & $-8,397$ & $-8,547$ & $-10,046$ & $-10,481$ & $-11,497$ & $-11,994$ & $-13,036$ & $-14,236$ \\
\hline Services (net) & 983 & 1,691 & 2,467 & 2,967 & 2,898 & 3,296 & 3,615 & 3,994 & 4,356 & 4,752 \\
\hline Receipts & 7,009 & 8,411 & 8,974 & 10,238 & 10,671 & 11,569 & 12,508 & $\uparrow 3,533$ & 14,602 & 15,750 \\
\hline Payments & $-6,026$ & $-6,720$ & $-6,508$ & $-7,272$ & $-7,773$ & $-8,273$ & $-8,893$ & $-9,539$ & $-10,247$ & $-10,998$ \\
\hline Income (net) & $-1,231$ & $-1,518$ & $-1,061$ & -991 & -993 & -707 & -629 & -636 & -638 & -636 \\
\hline o.w. interest on government debt & -525 & -502 & -696 & -457 & -522 & -535 & -540 & -549 & -563 & -575 \\
\hline Current transfers (net) & 2,417 & 2,902 & 2,196 & 2,231 & 2,393 & 1,784 & 1,892 & 2,016 & 2,143 & 2,205 \\
\hline Of which: Stockholm and Paris III grants & $\ldots$ & $\ldots$ & $\ldots$ & 937 & 873 & 509 & 292 & 120 & 0 & 0 \\
\hline Capital and financial account & 5,653 & 4,204 & 2,020 & 3.596 & $-1,885$ & 3,944 & 872 & 2,275 & 2,590 & 2,726 \\
\hline Direct investment (net) & 1,722 & 2,333 & 2,629 & 2,724 & 1,800 & 5,845 & 2,100 & 2,300 & 2,500 & 2,700 \\
\hline Portfolio investment, loans, other capital & 3,931 & 1,871 & -609 & 872 & $-3,685$ & $-1,901$ & $-1,228$ & -25 & 90 & 26 \\
\hline Government (net) & -773 & -87 & 297 & 767 & -342 & -496 & 32 & 33 & -4 & -499 \\
\hline Eurobonds (net) & -475 & 361 & 292 & 630 & -37 & -618 & 10 & 9 & -68 & -404 \\
\hline Treasury bills (net) & -268 & -484 & 51 & -33 & -86 & -78 & 26 & 6 & 5 & 7 \\
\hline Loans (net) & -29 & 36 & -46 & 170 & -219 & 200 & -3 & 17 & 59 & -102 \\
\hline BdL 1/ & -18 & -3 & 474 & 1,507 & 109 & -18 & -550 & -69 & -88 & -69 \\
\hline Of which: IMF (net) 2 I & 0 & 0 & 0 & 0 & 77 & 0 & 0 & -19 & -38 & -19 \\
\hline Banks (net) 31 & 3,845 & 1,293 & 469 & $-1,446$ & -167 & -866 & -443 & -247 & -248 & -250 \\
\hline Foreign assets of banks 4 / & -48 & $-2,706$ & -90 & $-2,516$ & -333 & $-1,658$ & -663 & -663 & -663 & -663 \\
\hline Non-resident deposits $3 /$ & 3,892 & 3,999 & 560 & 1,071 & 166 & 792 & 221 & 416 & 415 & 413 \\
\hline Non-bank private sector (net) & 876 & 668 & $-1,849$ & 44 & $-3,285$ & -522 & -268 & 259 & 430 & 844 \\
\hline Errors and omissions & 2 & $-1,523$ & 533 & -306 & 0 & 0 & 0 & 0 & 0 & 0 \\
\hline Overall balance & 3,030 & -696 & 36 & 1,742 & $-4,481$ & 1,449 & $-1,628$ & 394 & 839 & 975 \\
\hline Financing & $-3,030$ & 696 & -36 & $-1,742$ & 4,481 & $-1,449$ & 1,628 & -394 & -839 & -975 \\
\hline Official reserves (- increase) & $-5,125$ & 696 & -36 & $-1,742$ & 912 & $-2,699$ & 1,471 & -547 & -839 & -975 \\
\hline Exceptional financing & 2,095 & 0 & 0 & 0 & 3.569 & 1.250 & 157 & 153 & 0 & 0 \\
\hline \multicolumn{11}{|l|}{ Memorandum items: } \\
\hline Current account balance (in percent of GDP) & -13.3 & -15.8 & -11.7 & -6.8 & -11.0 & -10.0 & -9.4 & -6.6 & -5.7 & -5.4 \\
\hline excluding official transfers (in percent of GDP) & -13.9 & -16.3 & -12.4 & -11.6 & -15.3 & -12.6 & -11.0 & -7.5 & -6.2 & -5.8 \\
\hline \multicolumn{11}{|l|}{ Gross official reserves (excl. gold, end-year) $5 /$} \\
\hline in millions of U.S. dollars & 10,271 & 9,575 & 9,611 & 11,353 & 10,440 & 13,139 & 11,668 & 12,215 & 13,054 & 14,030 \\
\hline in months of next year's g\&s imports & 8.1 & 7.7 & 7.3 & 7.6 & 6.7 & 7.7 & 6.5 & 6.3 & 6.2 & 6.2 \\
\hline in percent of short-term external debt $6 /$ & 35.6 & 27.1 & 28.4 & 31.9 & 32.5 & 40.1 & 35.9 & 36.5 & 37.1 & 40.5 \\
\hline in percent of short-term foreign currency debt $7 /$ & 26.8 & 20.4 & 19.3 & 20.9 & 19.9 & 23.7 & 20.8 & 21.0 & 21.1 & 22.8 \\
\hline net of foreign assets of commercial banks & 34.3 & 26.7 & 25.9 & 28.5 & 28.1 & 34.3 & 30.3 & 30.3 & 30.5 & 32.4 \\
\hline \multicolumn{11}{|l|}{ External debt (end-year) $6 /$} \\
\hline in percent of GDP & 174.8 & 188.2 & 190.8 & 199.7 & 182.6 & 175.1 & 162.8 & 155.1 & 147.7 & 140.3 \\
\hline in percent of g\&s exports & 396.0 & 384.5 & 363.3 & 346.7 & 312.4 & 287.9 & 260.6 & 241.9 & 225.1 & 209.3 \\
\hline Government external debt (in percent of GDP) & 36.8 & 34.0 & 35.8 & 37.3 & 41.7 & 40.1 & 38.0 & 35.9 & 33.5 & 29.7 \\
\hline \multicolumn{11}{|l|}{ Govemment external debt service } \\
\hline in millions of U.S. dollars & 1,669 & 2,218 & 3,209 & 1,753 & 1,528 & 1,618 & 1,847 & 1,517 & 1,673 & 2,446 \\
\hline in percent of g\&s exports & 19.1 & 21.2 & 28.5 & 13.5 & 11.1 & 10.7 & 11.1 & 8.3 & 8.4 & 11.2 \\
\hline GDP (in millions of U.S. dollars) & 19,802 & 21,369 & 21,428 & 22.622 & 23,646 & 24,964 & 26,609 & 28,498 & 30,521 & 32,688 \\
\hline
\end{tabular}

Sources: Lebanese authorities; BIS; and IMF staff estimates and projections.

$1 /$ Change in the foreign liabilities of the BdL.

2/ For the period beyond March 2007, it is assumed that Fund purchases proceed as programmed and that repurchases are made on an obligation basis.

$3 /$ Differs from banks' reported data, to include estimated deposit flows by Lebanese nationals living abroad but classified as residents.

4/ Net of non-deposit foreign liabilities.

5/ Excludes Eurobonds and encumbered reserves.

$6 /$ Includes all banking deposits held by non-residents, including estimated deposits of Lebanese nationals living abroad but classified as residents.

7/ Includes private seclor foreign currency deposits in commercial banks. 
Table 10. Lebanon: External Financing Requirements and Sources, 2003-08

(In millions of U.S. dollars)

\begin{tabular}{|c|c|c|c|c|c|c|}
\hline & $\begin{array}{r}2003 \\
\text { Act. }\end{array}$ & $\begin{array}{r}2004 \\
\text { Act. }\end{array}$ & $\begin{array}{r}2005 \\
\text { Act. }\end{array}$ & $\begin{array}{c}2006 \\
\text { Prel. Act. } \\
\end{array}$ & $\begin{array}{l}2007 \\
\text { Proj. }\end{array}$ & $\begin{array}{l}2008 \\
\text { Proj. }\end{array}$ \\
\hline 1. Gross financing requirements & $-32,198$ & $-33,363$ & $-40,491$ & $-39,509$ & $-39,288$ & $-39,042$ \\
\hline External current account balance (exc. official transfers) & $-2,759$ & $-3,488$ & $-2,656$ & $-2,625$ & $-3,608$ & $-3,143$ \\
\hline $\begin{array}{l}\text { Debt amortization } \\
\text { Medium and long term debt } \\
\text { Short-term debt } 1 / 2 /\end{array}$ & $\begin{array}{r}-24,315 \\
-1,143 \\
-23,171\end{array}$ & $\begin{array}{r}-30,571 \\
-1,716 \\
-28,855\end{array}$ & $\begin{array}{r}-37,799 \\
-2,513 \\
-35,287\end{array}$ & $\begin{array}{r}-35,142 \\
-1,296 \\
-33,846\end{array}$ & $\begin{array}{r}-36,590 \\
-1,006 \\
-35,584\end{array}$ & $\begin{array}{r}-33,195 \\
-1,083 \\
-32,112\end{array}$ \\
\hline $\begin{array}{l}\text { Repayment of arrears } \\
\text { Gross reserves accumulation } \\
\text { IMF repurchases and repayments }\end{array}$ & $\begin{array}{r}0 \\
-5,125 \\
0\end{array}$ & $\begin{array}{r}0 \\
696 \\
0\end{array}$ & $\begin{array}{r}0 \\
-36 \\
0\end{array}$ & $\begin{array}{r}0 \\
-1,742 \\
0\end{array}$ & $\begin{array}{r}0 \\
912 \\
-3\end{array}$ & $\begin{array}{r}0 \\
-2,699 \\
-4\end{array}$ \\
\hline 2. Available financing & 32,198 & 33,363 & 40,491 & 39,509 & 40,161 & 39,551 \\
\hline Official grants & 134 & 111 & 139 & 1,076 & 1,012 & 648 \\
\hline Foreign direct investment (net) & 1,722 & 2,333 & 2,629 & 2,724 & 1,800 & 5,845 \\
\hline $\begin{array}{l}\text { Debt financing from private creditors } \\
\text { Medium- and long-term financing } \\
\text { Short-term financing } 2 /\end{array}$ & $\begin{array}{r}21,025 \\
859 \\
20,166\end{array}$ & $\begin{array}{r}28,335 \\
701 \\
27,634\end{array}$ & $\begin{array}{r}38,277 \\
885 \\
37,391\end{array}$ & $\begin{array}{r}34,428 \\
1,189 \\
33,239\end{array}$ & $\begin{array}{r}42,407 \\
2,227 \\
40,181\end{array}$ & $\begin{array}{r}35,199 \\
1,279 \\
33,920\end{array}$ \\
\hline Official creditors $3 /$ & 681 & 278 & 428 & 609 & 1,894 & 1,165 \\
\hline IMF & 0 & 0 & 0 & 0 & 77 & 0 \\
\hline Accumulation of arrears (exceptional) & 0 & 0 & 0 & 0 & 0 & 0 \\
\hline Other flows 4/ & 8,636 & 2,307 & -982 & 672 & $-7,029$ & $-3,306$ \\
\hline $\begin{array}{l}\text { 3. Financing gap } \\
\text { Expected disbursements of grants from donors }\end{array}$ & $\begin{array}{c}0 \\
\cdots\end{array}$ & $\begin{array}{r}0 \\
\cdots\end{array}$ & $\begin{array}{l}0 \\
\cdots\end{array}$ & $\begin{array}{c}0 \\
\cdots\end{array}$ & $\begin{array}{l}873 \\
873\end{array}$ & $\begin{array}{l}509 \\
509\end{array}$ \\
\hline 4. Residual Financing Gap & 0 & 0 & 0 & 0 & 0 & 0 \\
\hline
\end{tabular}

Sources: Data provided by the Lebanese authorities; and IMF staff estimates and calculations.

$1 /$ Original maturity of less than 1 year. Stock at the end of the previous period.

$2 /$ Includes non-resident deposits held at commercial banks.

$3 /$ Only loans.

4/ Includes all other net financial flows, and errors and omissions. 


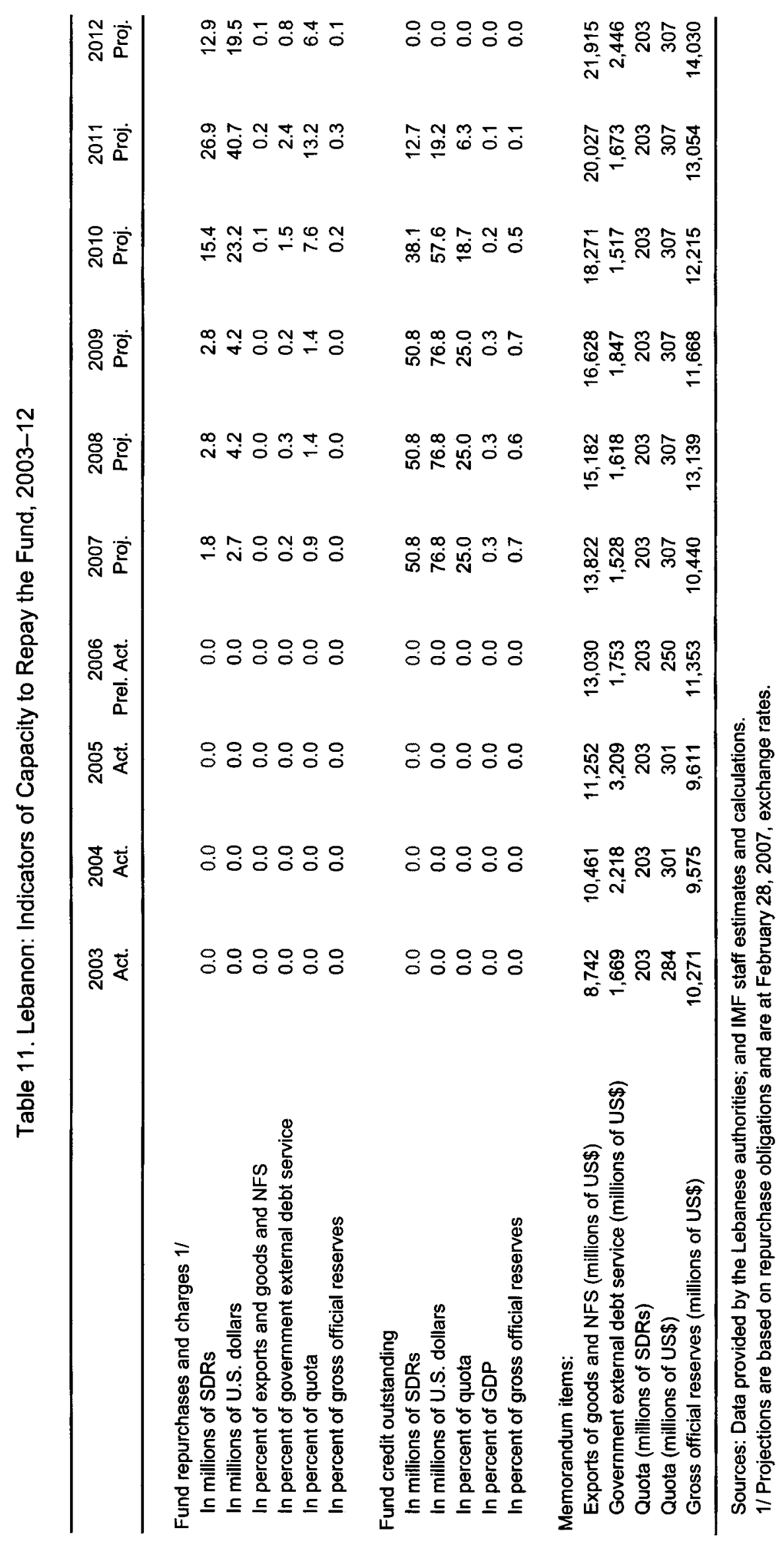


Table 12. Lebanon: Indicators of Financial and External Vulnerability, 2002-06

\begin{tabular}{|c|c|c|c|c|c|}
\hline & $\begin{array}{r}2002 \\
\text { Act. }\end{array}$ & $\begin{array}{r}2003 \\
\text { Act. }\end{array}$ & $\begin{array}{r}2004 \\
\text { Act. }\end{array}$ & $\begin{array}{r}2005 \\
\text { Act. }\end{array}$ & $\begin{array}{l}2006 \\
\text { Prel. Act. }\end{array}$ \\
\hline \multicolumn{6}{|l|}{ Monetary and financial indicators } \\
\hline Broad money, M5 (annual percentage change) & 6.4 & 15.5 & 12.3 & 3.5 & 6.4 \\
\hline Private-sector credit (annual percentage change) & 2.5 & 0.3 & 5.2 & 1.9 & 6.0 \\
\hline Broad money, M5 (in millions of U.S. dollars) & 43,848 & 50,652 & 56,871 & 58,852 & 62,606 \\
\hline \multicolumn{6}{|l|}{ Public finance indicators } \\
\hline Overall fiscal balance (in millions of U.S. dollars) & $-2,607$ & $-2,632$ & $-1,853$ & $-1,811$ & $-2,523$ \\
\hline In percent of GDP & -13.9 & -13.3 & -8.7 & -8.5 & -11.2 \\
\hline In percent of government revenue & -67.2 & -60.1 & -37.3 & -36.9 & -44.8 \\
\hline Interest payments on debt (in millions of U.S. dollars) & 3,127 & 3,280 & 2,602 & 2,263 & 2,907 \\
\hline In percent of GDP & 16.7 & 16.6 & 12.2 & 10.6 & 13.6 \\
\hline In percent of government revenue & 80.6 & 74.9 & 52.4 & 46.0 & 51.6 \\
\hline Nominal GDP (in millions of U.S. dollars) & 18,717 & 19,802 & 21,369 & 21,428 & 22,622 \\
\hline \multirow[t]{2}{*}{ Government revenue, excluding grants (in millions of U.S. dollars) } & 3,880 & 4,377 & 4,966 & 4,914 & 5,631 \\
\hline & \multicolumn{4}{|c|}{ (In percent) } & \\
\hline \multicolumn{6}{|l|}{ Banking-sector indicators } \\
\hline Problem loans/total loans $1 /$ & 27.2 & 29.3 & 27.2 & 24.8 & 22.7 \\
\hline Problem loans/total loans (net of provisions and unearned interest) $1 /$ & 12.4 & 12.8 & 10.6 & 9.1 & 7.3 \\
\hline Provisions against problem loans/problem loans $1 /$ & 42.4 & 46.3 & 46.1 & 49.0 & 52.7 \\
\hline Capital adequacy ratio & 19.4 & 22.3 & 21.2 & 22.9 & n.a. \\
\hline \multirow[t]{2}{*}{ Credit to the private sector (in percent of GDP) } & 81.4 & 77.8 & 75.7 & 76.7 & 76.9 \\
\hline & \multicolumn{4}{|c|}{ (In millions of U.S. dollars) } & \\
\hline \multicolumn{6}{|l|}{ Debt indicators } \\
\hline Gross public debt & 30,727 & 33,381 & 35,861 & 38,464 & 40,466 \\
\hline In percent of government revenue & 791.8 & 762.6 & 722.1 & 782.8 & 718.6 \\
\hline In percent of GDP & 164.2 & 168.6 & 167.8 & 179.5 & 178.9 \\
\hline of which: foreign currency & 13,943 & 15,575 & 18,368 & 19,133 & 20,430 \\
\hline In percent of GDP & 74.5 & 78.7 & 86.0 & 89.3 & 90.3 \\
\hline Gross public debt held by the market & 26,239 & 22,526 & 24,899 & 26,681 & 29,604 \\
\hline In percent of GDP & 140.2 & 113.8 & 116.5 & 124.5 & 130.9 \\
\hline External debt $2 /$ & 27,934 & 34,621 & 40,222 & 40,877 & 45,179 \\
\hline In percent of GDP & 149.2 & 174.8 & 188.2 & 190.8 & 199.7 \\
\hline External public debt & 5,906 & 7,483 & 7,448 & 8,327 & 10,600 \\
\hline In percent of GDP & 31.6 & 37.8 & 34.9 & 38.9 & 46.9 \\
\hline Short-term external public debt $3 /$ & 1,143 & 1,716 & 2,513 & 1,296 & 1,006 \\
\hline Short-term foreign currency public debt $3 /$ & 1,645 & 1,770 & 2,452 & 2,914 & 1,798 \\
\hline Short-term external debt $2 / 3 /$ & 23,171 & 28,855 & 35,287 & 33,846 & 35,584 \\
\hline Short-term foreign currency debt $3 / 4 /$ & 34,391 & 38,318 & 46,875 & 49,783 & 54,209 \\
\hline Total foreign currency deposits (resident and non-resident) $2 /$ & 29,682 & 32,266 & 38,505 & 41,734 & 46,319 \\
\hline \multicolumn{6}{|l|}{ International reserves } \\
\hline Gross international reserves 5/ & 5,147 & 10,271 & 9,575 & 9,611 & 11,353 \\
\hline In percent of short-term external debt & 22.2 & 35.6 & 27.1 & 28.4 & 31.9 \\
\hline In percent of s.t. ext. debt, plus s.t. domestic public debt in foreign currency & 21.1 & 34.4 & 26.2 & 27.0 & 31.0 \\
\hline In percent of s.t. ext. debt and residents' f. c. deposits less banks' foreign assets & 20.1 & 34.3 & 26.7 & 25.9 & 28.5 \\
\hline Gross international reserves and commercial banks' foreign assets & 13,417 & 18,589 & 20,599 & 20,725 & 24,983 \\
\hline In percent of short-term external debt & 57.9 & 64.4 & 58.4 & 61.2 & 70.2 \\
\hline \multirow[t]{2}{*}{ In percent of short-term foreign currency debt 4/ } & 39.0 & 48.5 & 43.9 & 41.6 & 46.1 \\
\hline & \multicolumn{4}{|c|}{ (In millions of U.S. dollars) } & \\
\hline \multicolumn{6}{|l|}{ External current account indicators } \\
\hline Merchandise exports, f.o.b. & 1,210 & 1,733 & 2,050 & 2,278 & 2,792 \\
\hline Annual percentage change & 37.5 & 43.2 & 18.3 & 11.1 & 22.5 \\
\hline Merchandise imports, f.o.b. & 5,910 & 6,528 & 8,502 & 8,397 & 8,547 \\
\hline Annual percentage change & -13.1 & 10.4 & 30.3 & -1.2 & 1.8 \\
\hline External current account balance & $-2,669$ & $-2,625$ & $-3,377$ & $-2,517$ & $-1,549$ \\
\hline In percent of GDP & -14.3 & -13.3 & -15.8 & -11.7 & -6.8 \\
\hline In percent of exports of goods and services & -34.7 & -30.0 & -32.3 & -22.4 & -11.9 \\
\hline
\end{tabular}

Sources: Lebanese authorities; Bank for International Settlements; and Fund staff estimates and projections.

$1 / 2006$ refers to November data.

2/ Includes estimates for public debt and banking deposits held by non-residents, and non-resident claims on the nonfinancial sector.

3/ On a remaining maturity basis (scheduled amortization over the next year).

4/ Short-term foreign currency debt of the public sector and the banking sector plus external debt of the nonbank sector.

5/ Excludes gold and encumbered assets. 
Table 13. Lebanon: Banking Sector Financial Soundness Indicators, 2002-06

\begin{tabular}{|c|c|c|c|c|c|}
\hline & $\begin{array}{r}2002 \\
\text { Act. }\end{array}$ & $\begin{array}{r}2003 \\
\text { Act. }\end{array}$ & $\begin{array}{r}2004 \\
\text { Act. }\end{array}$ & $\begin{array}{r}2005 \\
\text { Act. }\end{array}$ & $\begin{array}{r}2006 \\
\text { Prel. Act. }\end{array}$ \\
\hline & \multicolumn{5}{|c|}{ (In percent, unless otherwise specified) } \\
\hline Assets (in millions of U.S. dollars) & 54,587 & 61,589 & 70,595 & 73,565 & 78,531 \\
\hline \multicolumn{6}{|l|}{ Capital } \\
\hline Capital adequacy ratio & 19.4 & 22.3 & 21.2 & 22.9 & n.a. \\
\hline Capital to asset ratio & 7.0 & 6.9 & 6.8 & 7.5 & 8.6 \\
\hline \multicolumn{6}{|l|}{ Asset quality } \\
\hline Net problem loans/net total loans & 12.4 & 12.8 & 10.6 & 9.1 & 7.3 \\
\hline Provisions against problem loans/problem loans & 42.4 & 46.3 & 46.1 & 49.0 & 52.7 \\
\hline Total provisions/problem loans & 51.2 & 53.1 & 57.3 & 63.3 & 70.0 \\
\hline \multicolumn{6}{|l|}{ Asset concentration } \\
\hline Share of claims on government & 33.6 & 23.2 & 23.6 & 25.2 & 27.2 \\
\hline of which: T-bills & 20.5 & 12.6 & 10.6 & 11.9 & 13.7 \\
\hline of which: Eurobonds & 13.0 & 10.6 & 13.0 & 13.3 & 14.0 \\
\hline Share of claims on BdL & 15.2 & 31.3 & 28.6 & 29.9 & 27.0 \\
\hline of which: Certificates of Deposit & 0.7 & 16.1 & 12.0 & 13.4 & 11.7 \\
\hline Share of claims on private sector & 28.8 & 25.2 & 23.5 & 23.1 & 22.8 \\
\hline Share of claims on nonresidents & 16.7 & 14.4 & 17.6 & 16.4 & 16.8 \\
\hline of which: foreign banks & 15.7 & 13.4 & 16.3 & 14.9 & 14.7 \\
\hline Net foreign currency assets as percent of capital & 24.9 & 22.0 & 23.3 & 18.3 & 17.4 \\
\hline \multicolumn{6}{|l|}{ Earnings } \\
\hline Average return on assets (post tax) & 0.6 & 0.7 & 0.7 & 0.7 & 0.9 \\
\hline Average return on equity (post tax) & 9.2 & 10.9 & 9.3 & 11.0 & 10.6 \\
\hline Net interest margin 1/ & 2.1 & 2.1 & 1.7 & 1.8 & 1.6 \\
\hline \multicolumn{6}{|l|}{ Liquidity } \\
\hline Net liquid assets/total assets & 41.3 & 49.1 & 43.2 & 45.2 & 43.5 \\
\hline Net liquid assets/short-term liabilities & 49.2 & 56.6 & 57.0 & 52.8 & 51.6 \\
\hline Private sector deposits/assets & 69.6 & 68.2 & 66.8 & 67.6 & 67.9 \\
\hline Nonresident deposits/assets & 11.6 & 12.9 & 14.1 & 13.5 & 12.3 \\
\hline \multicolumn{6}{|l|}{ Other indicators $2 l$} \\
\hline Change in assets (12 month, in percent) & 9.5 & 12.8 & 15.2 & 4.2 & 6.8 \\
\hline Change in private sector credit ( 12 month, in percent) & 2.5 & 0.3 & 5.2 & 1.9 & 6.0 \\
\hline Change in deposits (12 month, in percent) & 6.4 & 15.5 & 12.3 & 3.5 & 6.4 \\
\hline FC deposits/total deposits $3 /$ & 69.4 & 66.2 & 70.1 & 73.2 & 76.2 \\
\hline FC loans/total loans & 86.3 & 87.0 & 86.4 & 86.6 & 86.9 \\
\hline FC loans/GDP & 67.1 & 62.8 & 62.0 & 62.8 & 66.2 \\
\hline Exports of goods and services/GDP & 40.1 & 42.7 & 49.0 & 54.1 & 48.5 \\
\hline \multicolumn{6}{|l|}{ Memorandum items: } \\
\hline LL deposit rate (average) & 10.3 & 7.8 & 7.1 & 7.7 & 7.5 \\
\hline LL loan rate (average) & 16.6 & 13.4 & 10.8 & 10.6 & 10.3 \\
\hline FC deposit rate (average) & 4.2 & 3.6 & 3.3 & 3.7 & 4.4 \\
\hline FC loan rate (average) & 10.0 & 9.0 & 8.3 & 8.2 & 8.5 \\
\hline Government's 2-year T-bill rate (marginal) & 13.6 & 8.0 & 7.9 & 8.5 & 8.7 \\
\hline Spread over 6-month USD Libor & 11.7 & 6.8 & 6.1 & 4.8 & 3.4 \\
\hline Government's Eurobond rate (marginal) & 7.9 & 7.0 & 7.0 & 7.5 & 7.5 \\
\hline Spread over 5 year U.S. note & 4.0 & 4.0 & 3.5 & 2.7 & 1.8 \\
\hline GDP (in millions of U.S. dollars) & 18,717 & 19,802 & 21,369 & 21,428 & 22,395 \\
\hline
\end{tabular}

Sources: Banque du Liban, Banking Control Commission; and staff estimates.

$1 / 2006$ figure is the August net interest margin annualized.

2/ FC and LL stand for "foreign currency" and "Lebanese pound", respectively.

3/ FC deposits of residents and nonresidents as a share of total deposits of residents and nonresidents. 


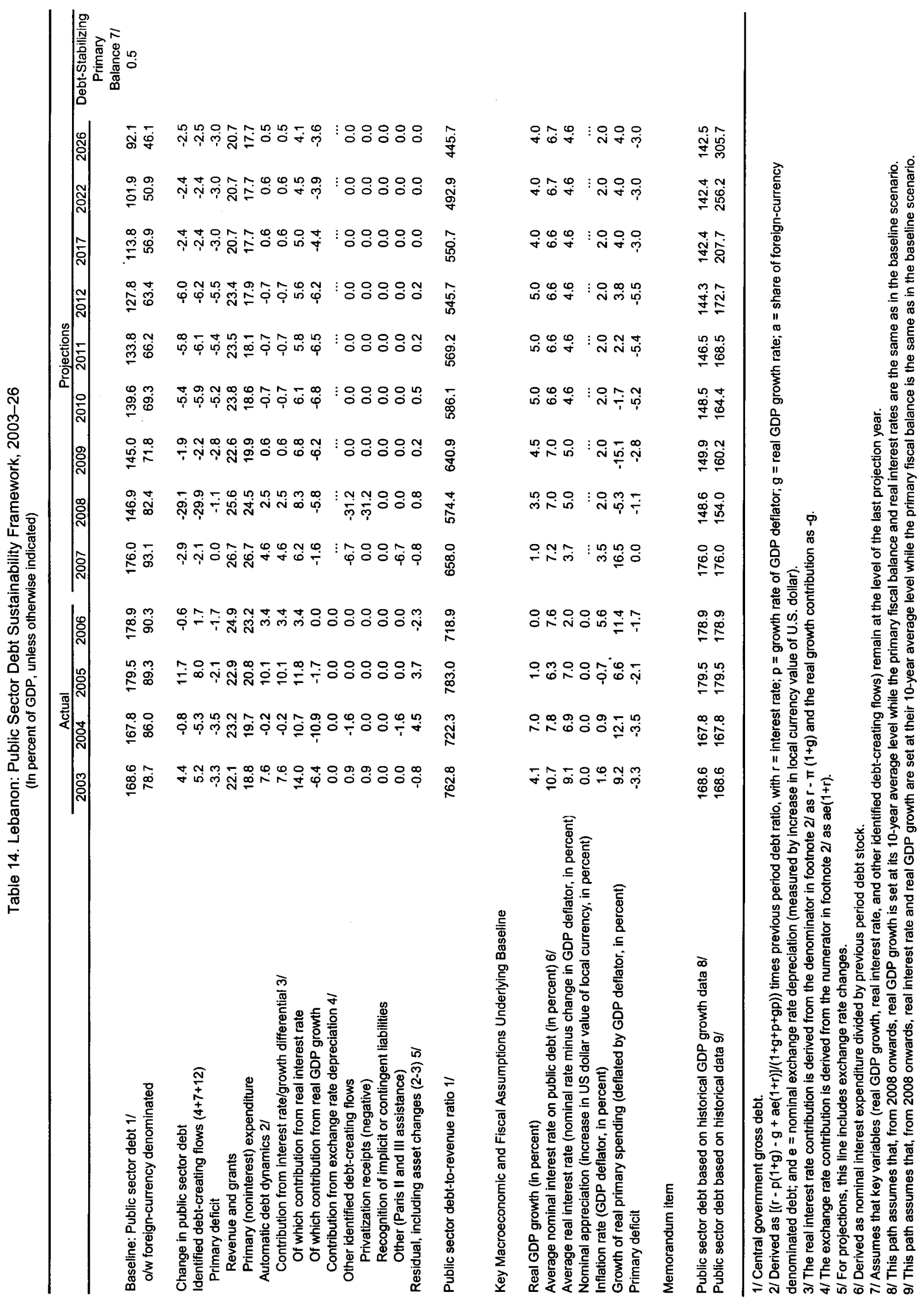




\title{
APPENDIX. LEBANON: LETTER OF INTENT
}

March 30, 2007

\author{
Mr. Rodrigo de Rato \\ Managing Director \\ International Monetary Fund \\ $70019^{\text {th }}$ Street N.W. \\ Washington, D.C. 20431
}

Dear Mr. de Rato:

In 2006, the government had made significant progress in preparing a comprehensive five-year reform program that was to be presented to a Donors Conference in Beirut. At the time of the outbreak of the conflict with Israel, the government was close to adopting this program, after extensive consultations with domestic and international stakeholders.

The conflict not only inflicted large human and economic costs to Lebanon, but also set back the timeline of the government reform agenda. However, while the war and its devastating consequences shifted the government's short-term attention to immediate relief and reconstruction efforts, the government remained fully committed to pursue economic reforms and consolidate the public accounts. Indeed, the urgency of implementing the program was amplified by the conflict, which put further strain on the public finances and the management of the large public debt.

For these reasons, in the months following the conflict the government worked to adapt the program to the new circumstances with the intention of launching the reform agenda in 2007. The overall structure of the program has remained intact. However, particular attention was accorded to the timing of the reform measures, taking into consideration the difficulties to implement up-front reforms in the post conflict situation. The program also took into account the impact of the conflict on the macroeconomic situation and the heightened social needs of the population. On the fiscal front, the reform program aims at improving the fiscal situation, in particular through an intensification of the revenue effort starting in 2008, the rationalization of expenditures, and at improving efficiency and competitiveness through energy sector reform and a privatization plan that relates to the telecommunications sector. Fiscal consolidation will be supported by a strict monetary policy, aimed at maintaining price stability and supporting the exchange rate peg. 
The reform program complements these macroeconomic policies with a social sector reform plan aimed at alleviating poverty, reducing regional income disparities, and improving education and health indicators; and with a growth-enhancing structural reform plan that will strengthen the business climate and the competitiveness of Lebanese business.

In the context of the program, the government remains committed to an open trade and exchange system, and is taking steps to become a full member of the World Trade Organization. Looking forward, the government will avoid imposing restrictions on payments and transfers for international transactions, to introduce or intensify trade restrictions for balance of payments purposes, or resort to multiple currency practices.

The government received the strong support for its reform program from the international donor community at the donors' conference chaired by President Chirac in Paris on January 25, 2007. The Paris conference generated considerable financial support toward reducing the government's debt burden. Some donors expressed support for IMF monitoring of the implementation of certain measures proposed in the reform program.

The government of Lebanon intends to actively pursue its reform program and, in order to support the implementation of the measures envisaged in the program for 2007, hereby requests access to the IMF's policy for emergency post-conflict assistance (EPCA), in an amount equivalent to SDR 50.75 million, or 25 percent of quota. In view of this request, and as described in the attached tables, the government has revised the program's macroeconomic projections on the basis of the latest economic developments, and has selected key macroeconomic indicators and structural measures that it intends to monitor closely and report on during 2007.

Some of the measures that are laid out in this request are subject to the settlement of the current political stalemate and approval by Parliament. Given the sensitive nature of these measures, particularly in light of the destruction caused by the war and its social consequences, they are also contingent upon an internal consensus in the country.

A satisfactory implementation of the 2007 program under EPCA, a strengthening of capacity, also through technical assistance from the Fund, and an improvement of economic and social conditions, should allow the government to move, in 2008, to a more ambitious part of its five-year program. At that stage, the government intends to seek further support for its program through a stand-by arrangement from the Fund. 
The Government of Lebanon and Banque du Liban will provide the IMF with any available information it may request on the implementation of the program, consistent with the understandings reflected in the attached Technical Memorandum of Understanding, and will consult with Fund staff regarding any revision to the policies described in the government reform program.

Sincerely yours,

$/ \mathrm{s} /$

Jihad B. Azour

Minister of Finance
$/ \mathbf{s} /$

Riad T. Salamé

Governor

Banque du Liban

\section{Attachments}

I. Quantitative Indicative Targets

II. Monitorable Actions

III. Authorities' Medium-Term Reform Program Presented at the Paris III Conference

IV. Technical Memorandum of Understanding 


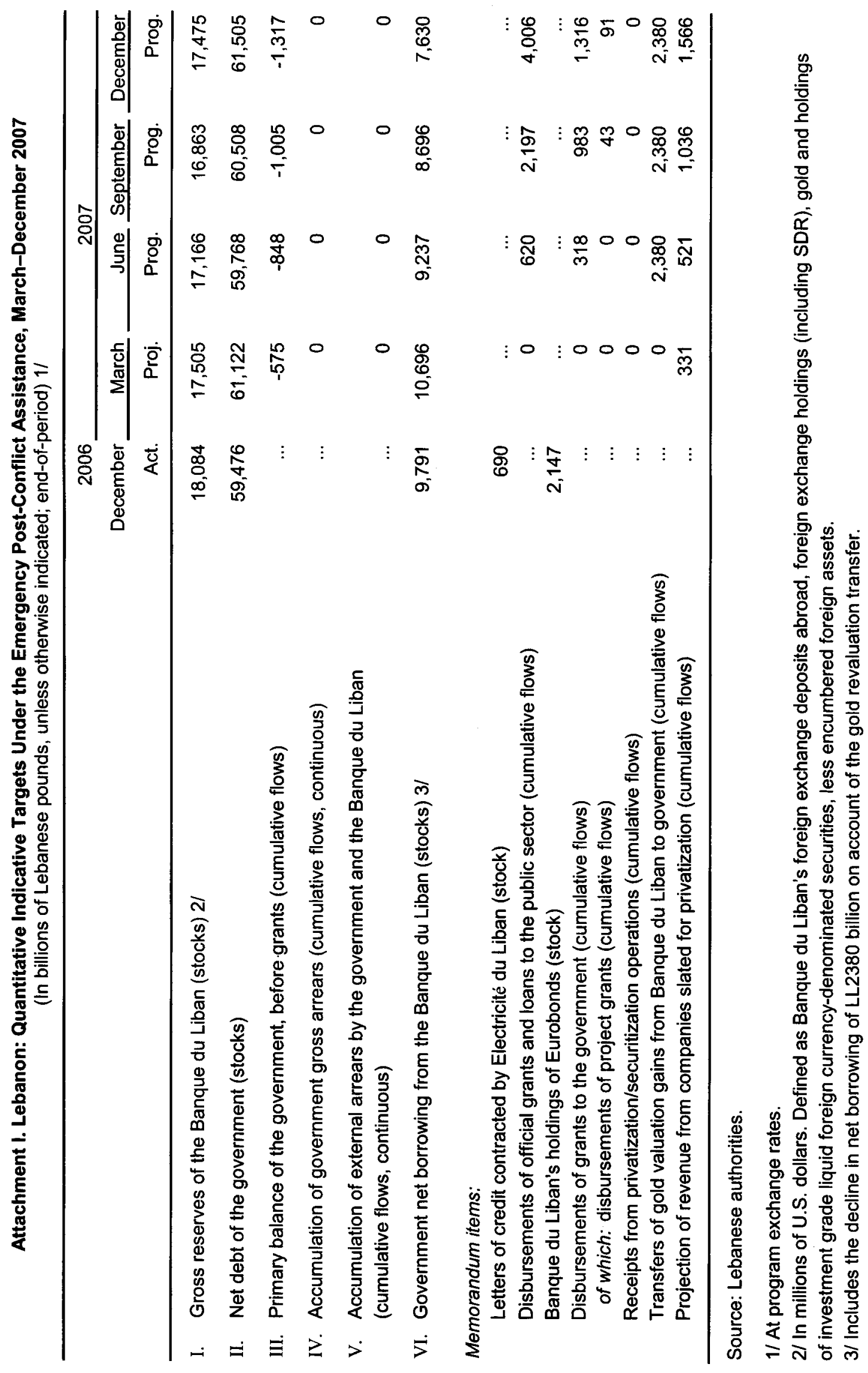


Attachment II. Lebanon: Monitorable ACtions

Monitorable Actions for the Period March-December 2007

\begin{tabular}{|c|c|c|}
\hline Measure & Target Date & Implementation \\
\hline \multicolumn{3}{|l|}{ Fiscal } \\
\hline $\begin{array}{l}\text { Submit to Parliament a draft } 2007 \text { budget law consistent } \\
\text { with the targeted primary balance of the central } \\
\text { government, and including detailed HRC operations and } \\
\text { CDR foreign-financed expenditures. The budget law should } \\
\text { not allow budget carryovers for expenditures for which no } \\
\text { third-part liability already exists, and treasury advances of } \\
\text { more than one month after the fiscal year. }\end{array}$ & End-June 2007 & \\
\hline $\begin{array}{l}\text { Issue a Cabinet of Ministers decision setting a specific floor } \\
\text { on gasoline excise of } L L 300 \text { per liter of gasoline. }\end{array}$ & End-September 2007 & \\
\hline $\begin{array}{l}\text { Appoint auditor for NSSF accounts. Auditor to prepare an } \\
\text { audit plan of NSSF for 2001-06. }\end{array}$ & End-June 2007 & \\
\hline \multicolumn{3}{|l|}{ Power sector } \\
\hline $\begin{array}{l}\text { Appoint auditor for EdL accounts. Auditor to prepare an } \\
\text { audit plan of EdL for 2002-2006. Publish } 2001 \text { audit report. }\end{array}$ & End-June 2007 & \\
\hline \multicolumn{3}{|l|}{ Privatization } \\
\hline $\begin{array}{l}\text { Submit to Parliament the draft law to authorize the sale of } \\
\text { the mobile sector's assets and relevant operating licenses by } \\
\text { the government }\end{array}$ & End-June 2007 & \\
\hline $\begin{array}{l}\text { Issue an invitation for expression of interest (EOI) in } \\
\text { participating in the process of acquiring the licenses and } \\
\text { assets of the two mobile telephone companies (MICl and } \\
\text { MIC2) }\end{array}$ & End-December 2007 & \\
\hline
\end{tabular}


ATtACHMENT III. LebaNon: AUthoRITIES' MEdUM-TERM REFoRM PROGRAM Presented at The Paris III CONFEREnCe

January 02, 2007

Lebanese Republic

RECOVERY, RECONSTRUCTION, AND REFORM

“International Conference for Support to Lebanon"

25 January 2007

Paris 


\section{ABBREVIATIONS AND ACRONYMS}

BdL

BOT

CDR

EdL

EU

GDP

HCP

HRC

INSEE

LL

MEA

MOF

NGO

NSSF

PMO

SME

UN

UNDG

UNDP

UN MACC

VAT
Banque du Liban (Central Bank of Lebanon)

Build-Own-Transfer

Council for Development and Reconstruction

Eléctricité du Liban

European Union

Gross Domestic Product

Higher Council for Privatization

Higher Relief Commission

Institut National de la Statistique et des Etudes Economiques

Lebanese Pounds

Middle East Airlines

Ministry of Finance

Non-Governmental Organization

National Social Security Fund

Prime Minister's Office

Small and Medium Scale Enterprise

United Nations

United Nations Development Group

United Nations Development Program

United Nations Mine Action Coordination Center

Value Added Tax 


\section{"International Conference for Support to Lebanon" \\ RECOVERY, RECONSTRUCTION AND REFORM}

Contents
RECOVERY, RECONSTRUCTION AND REFORM

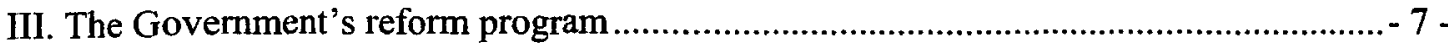

A. Growth-Enhancing Structural Reforms …...................................................... 9 -

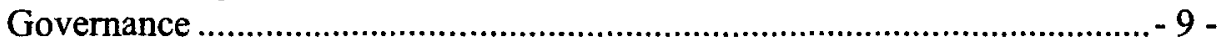

Financial Sector Reform and Debt Management...................................... 10 -

Improving the business environment .................................................... 11 -

B. Social Sector Reforms ................................................................................. 13

Main elements of the social plan ........................................................... 14 -

Specific social interventions ............................................................ 15 -

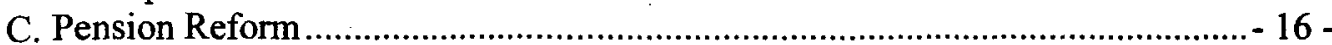

D. Fiscal Adjustment and Structural Reform....................................................... 17 -

Expenditure Measures.................................................................... - 17 -

Revenue Measures ............................................................................. 21 -

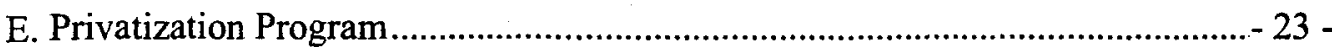

F. Monetary and Exchange Rate Policies ................................................... 25 -

G. Banking Sector's Contribution to the Domestic Effort ..................................... 25 -

IV. Medium-Term macroeconomic Framework ........................................................... 26 -

A. Macroeconomic scenario with domestic adjustment ...................................... 26 -

B. Macroeconomic Scenario with External Support............................................ 27 -

C. Implementation of the reform program ...................................................... 28 -

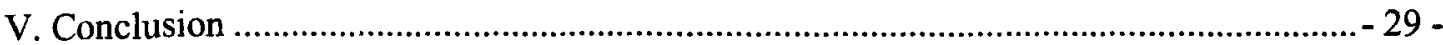




\section{Lebanese Republic}

\section{RECOVERY, RECONSTRUCTION AND REFORM}

\section{Preface}

In April 2005, Lebanon reclaimed its democracy and independence through a popular uprising triggered by the assassination of late Prime Minister Rafic Hariri. The international community rallied in support of Lebanon and, on the occasion of the Core Group meeting in New York in September 2005, vowed to support the new government and help Lebanon reduce its debt to sustainable levels so it can achieve growth, equity, and political stability.

Following that meeting, the Government prepared a comprehensive reform program that was to be presented to a Donors Conference in Beirut with a request for international support to complement the significant domestic effort that was envisaged in this program. Following extensive consultations with domestic and international stakeholders, the government was on the verge of adopting this program when Lebanon once again came under a massive Israeli attack.

The direct and indirect costs of the July 2006 war far exceed the capacity of any middle-income country to bear, let alone a country that has been saddled with a very large public debt and a country that had just come out of a major setback associated with the assassination of Prime Minister Hariri, which has left deep scars on the economic and political landscape. As a result, the burden of Lebanon's public debt-largely a legacy of the 1975-92 war and its aftermath-has become even heavier.

While the July war and its devastating consequences have shifted the government's short term attention to immediate major relief efforts and reconstruction needs, the government remains fully committed to pursue economic reforms, although some changes to the timetable and to the size of adjustment are needed. These changes are necessary to take into account the impact of the war on the macroeconomic situation, and to spread the burden of reform over time so as to give the suffering population a breathing space following this year's tragic events.

By the end of 2006, Lebanon's debt will be around US\$40.5 billion, or about $180 \%$ of GDP. Despite its resiliency, Lebanon cannot shoulder this price on its own. The re-phasing of some measures necessitated by the latest Israeli war will fail to achieve growth, equity, and debt sustainability in the absence of sizeable external support. Failure to achieve these objectives could well jeopardize Lebanon's broader goals of political and social stability, and the strengthening of its democracy. Lebanon is therefore hopeful that on the occasion of the "International Conference for Support to Lebanon", the international community will invest in Lebanon's future and democracy.

The Lebanese people are determined to build a strong state: a state which can reclaim the position of Lebanon as a haven of moderation where individual initiative and potential can be fulfilled. In our part of the world, Lebanon represents indeed a unique phenomenon of diversity, multiconfessionalism, democracy, and freedom of expression. We will strive to preserve and keep these ideals alive, but our friends' help is critical. We strongly believe that what happens in Lebanon will have repercussions that transcend Lebanon's borders and far exceed Lebanon's size. Our success in strengthening our democracy and building a strong economy is in the interest of all. 


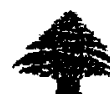 \\ Lebanese Republic \\ RECOVERY, RECONSTRUCTION AND REFORM
}

\begin{abstract}
Prelude
1. On the eve of July 12, 2006 the government of Lebanon was on the verge of adopting a comprehensive and bold economic reform program, which had set ambitious targets to address the main vulnerability of the Lebanese economy represented by the high level of public debt and to create the right environment for stimulating growth and creating employment. The program aimed also at mitigating the impact of adjustment on the less fortunate in the context of a well defined social action plan to be implemented in conjunction with economic reforms.
\end{abstract}

2. The July-August Israeli war shifted the government's attention to managing the humanitarian crisis, providing for massive rehabilitation needs, and rebuilding physical infrastructure especially that related to the country's productive capacity. Despite the difficult economic environment, the government remains fully committed to pursuing the kind of reform efforts included in the pre-war program. Naturally, the new reality on the ground imposes a different set of conditions and additional constraints that should be taken into account.

3. In this document, the government of Lebanon presents its reform program that aims primarily at stimulating growth, creating employment, and putting Lebanon's large public debt on a downward trend. The core elements of the program have not changed and were subject to wide and extensive consultations and discussions with domestic and international stakeholders prior to the July-August 2006 Israeli war.

4. The rest of the document is organized as follows. First, a brief background is presented along with an overview of recent developments. In the following section, a summary of the impact of the war will be presented as well as the financial assistance provided by the international community for immediate recovery and reconstruction needs. The government's reform program and the mediumterm outlook are then discussed in detail.

\section{BACKGROUND}

5. In mid-1970s, Lebanon was a democratic upper-middle income country prospering as a regional service center on the crossroads between Europe and the Middle East. Lebanon barely had any debt, reflecting prudent economic management and an economy that was driven mainly by a dynamic private sector and supported by a small size public sector. But Lebanon was also at the crossroads of international politics and regional conflicts, which have contributed to the outbreak of the war in 1975. Seventeen years of internal strife caused massive destruction. The UN estimated damage to physical assets at US $\$ 25$ billion, equivalent to about six to seven times the Gross Domestic Product (GDP) in 1990.

6. Prior to the outbreak of the civil war in 1975, Lebanon had a liberal, open, and dynamic economy led by a dominant and vibrant private sector. The fifteen years of hostilities, however, had a debilitating effect on the private sector which struggled to regain its previous role as an engine for growth in providing employment and basic services. Given this fact, and the enormous need for physical reconstruction coupled with an inability to raise sufficient revenues, the public sector 
resorted to borrowing to reverse the impact of hostilities and provide the basic platform for the private sector to regain its previous role. This led to a change in the basic nature of the Lebanese economy that saw a rise in the role and the size of the public sector.

7. In the early 1990s, the government undertook the task of reconstruction of public infrastructure, and of investing in social peace, including incorporating the war militia, and providing for resettlement and social assistance to the displaced. Also, the government had to deal with the social consequences of the continued Israeli occupation of the South notwithstanding the recurring Israeli aggressions that necessitated additional outlays for reconstruction and special allocations to the Council of the South. With an eroded revenue base and limited access to external financing, the government relied heavily on borrowing domestically to finance sharply rising budget deficits. Given the magnitude of destruction, the government had to provide all basic services including education, health, water, and electricity. While there was significant international support for reconstruction, primarily in the form of concessional loans, overall more than two-thirds of all capital expenditures during the $1990 \mathrm{~s}$ were funded by market borrowing at high interest rates. Total public sector interest payments were double the size of capital expenditures during the 1990s.

8. By 2000, when south Lebanon was liberated, except for Shebaa farms, essential reconstruction was substantially completed, and real per capita income had more than doubled from the 1990 level, but still stood a quarter below its pre-war level. However, with ballooning interest payments (absorbing over three-quarters of revenues), the overall fiscal deficit reached nearly $25 \%$ of GDP in 2000. Gross public debt, which a decade earlier stood at about US\$2 billion, had grown to US\$25 billion, equivalent to $150 \%$ of GDP, and the economy was stagnating.

\section{Developments DURING 2001-05}

9. Against the background of a vicious cycle of rising fiscal deficits and debt and a stagnating economy, the government of late Prime Minister Hariri that came back to office in late 2000 developed an economic reform program, for which it sought external support in the context of the Paris II Meeting in 2002. On that occasion, the international community provided US\$2.4 billion in direct financial support (non-project financing) ${ }^{\prime}$. This, combined with a financial contribution from Lebanon's financial sector (Banque du Liban and commercial banks ${ }^{2}$ ), and the effects that the external support had had on confidence in financial markets, resulted in a significant decline in interest rates.

\footnotetext{
' At a maturity of 15 years and an interest of $5 \%$.Three-quarters of the amount has a grace period of five years and the remainder has a grace period of 3 years.

${ }^{2}$ Government's debt to BdL was reduced by US $\$ 1.8$ billion and US $\$ 2.3$ billion in debt was converted into a long-term loan at concessional rates. Commercial banks subscribed to a two-year Government security at $0 \%$ interest in an amount equivalent to $10 \%$ of their deposits (US\$3.6 billion).
} 

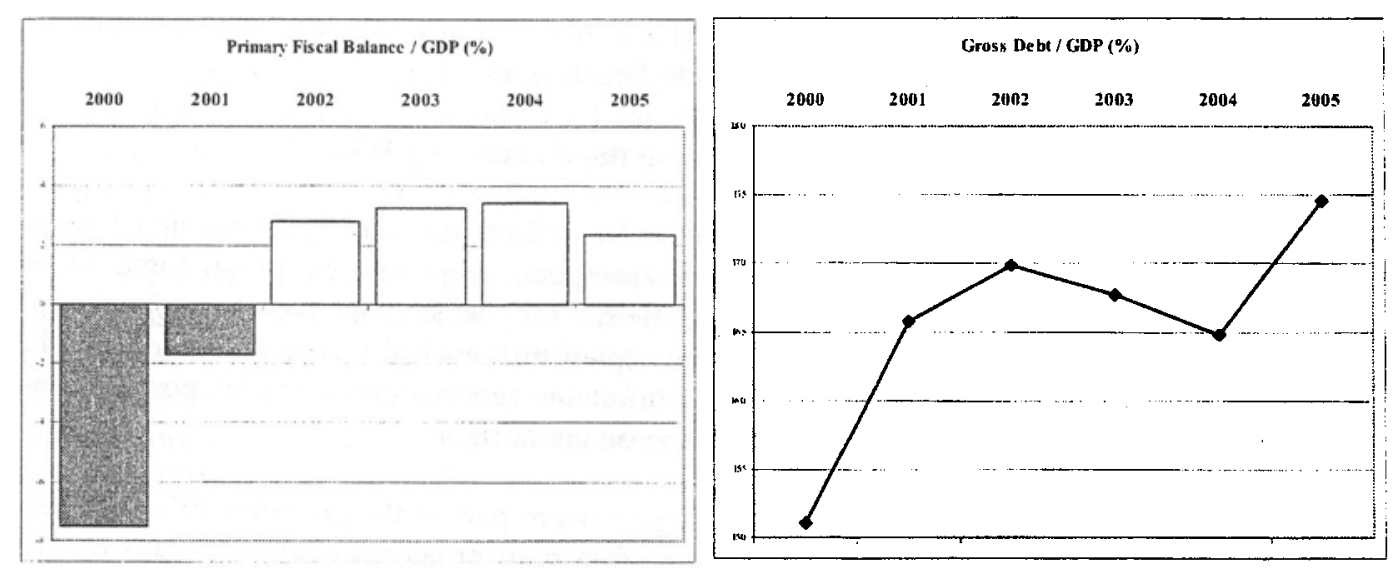

10. The financial package helped improve substantially the structure of the public debt and sharply reduced its cost. As a result, interest payments on the debt fell sharply from $17 \%$ of GDP in 2002 to about $10 \%$ of GDP in 2005. In 2004, macroeconomic performance exceeded all expectation with real growth reaching more than $7 \%$, the overall budget deficit declining to less than $8 \%$ of GDP (from $25 \%$ in 2000 ), and the primary budget surplus improving to $2.3 \%$ of GDP. The fiscal adjustment benefited considerably from the introduction of a Value Added Tax (VAT) in 2002. However, political squabbling prevented the full implementation of the structural components of the program, mainly privatization, and hence the full benefits of Paris 11 could not be realized. As a consequence, by end-2004, the level of gross public debt reached $165 \%$ of GDP and $175 \%$ of GDP by end-2005.

11. Strong economic performance in 2004 was cut short by political tensions that began in late 2004 with the extension of the Presidential mandate and the assassination of Prime Minister Hariri in February 2005. Economic activity declined sharply in the first half of the year to recover somewhat in the second half leading to an estimated real growth of $1 \%$ for the year. In addition, Lebanon was particularly hard hit by rising oil prices-given its heavy dependence on oil imports-for as much as $98 \%$ of its energy needs - of which about half is for power generation. This was compounded by the reduction in tax revenue since the government had

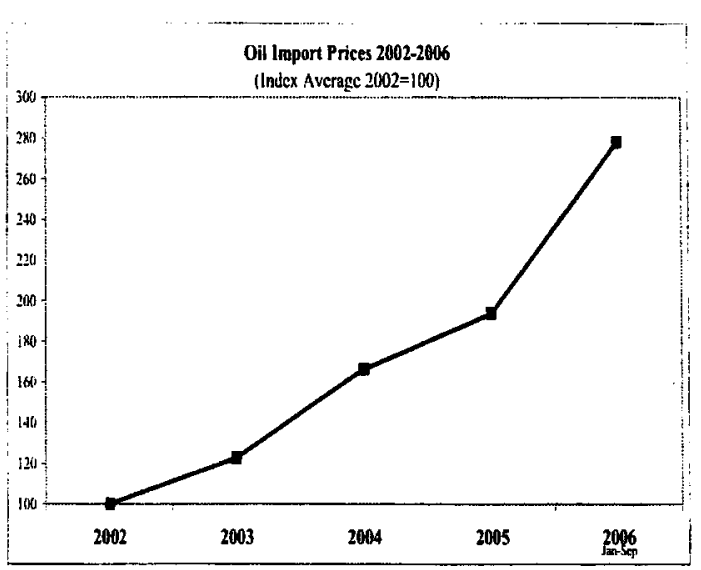
to cap the price of gasoline at its pre-May 2004 level in an attempt to shield the population from the sharp, and what was believed to be a temporary, increase in oil prices. At the same time, government transfers to the Electricity Company (EdL) increased substantially to cover the additional cost of fuel, and to cover the company's large technical and non-technical losses. In 2005, transfers to EdL from the budget reached about US\$650 million.

12. With parliamentary elections in mid-2005 and the formation of a new Cabinet with broad support in July 2005 , Lebanon was witnessing a new dawn. Despite the serious economic difficulties that followed the assassination of Prime Minister Hariri in February 2005, the Siniora government exerted significant efforts to redress the fiscal situation and rejuvenate the economy. Between July 2005 and June 2006, all public finance indicators were showing strong improvements: the real growth rate for 2006 was expected to reach 5-6\%, with a record high tourist season in the making and an 
increase of exports by more than $30 \%$; the primary surplus in the budget more than quadrupled; and the balance of payments, that showed a deficit of US\$1.5 billion in June 2005, recorded a surplus of US\$2.6 billion twelve months later on the eve of the July Israeli war.

\section{A. The Reform Program Prior to the Israeli July War}

13. The Siniora government prepared a comprehensive reform program that was about to be adopted before the July war. At that time, Lebanon's prospects were looking good. Signs of an excellent tourist season were very strong-flights to Beirut for the summer were over bookedforeign direct investment was on the rise, large private capital inflows had increased liquidity in the banking sector and helped the central bank build a comfortable international reserves position, and some early signs of a possible "brain drain" reversal were on the horizon.

14. Although the main elements of the reform program were part of the government's statement to parliament in July 2005, the Government decided to first seek broad national consensus on this program before holding the Beirut Conference. This was in order to ensure ownership of the reform program and therefore its implementation, and to avoid the situation that had arisen after the Paris Il Meeting when the government was unable to implement the full range of reforms (namely privatization). Given the nature, the magnitude and the scope of the reform measures of the actions that were envisaged, more time than had originally been anticipated was needed to complete the consensus building process, which was also complicated by some political tensions early in the year. The process was reaching its final stages when the war took place in July 2006.

15. The medium-term scenario under the pre-war program envisaged an average real GDP growth of about $5 \%$, strong fiscal adjustment with an improvement in the primary surplus by about $6 \%$ points of GDP during the years 2006-10, and privatization going forward, particularly the telecommunications sector. Yet, it showed that without external support, the debt to GDP ratio would decline from $175 \%$ at end- 2005 to about $135 \%$ by 2010 . But with interest payments still absorbing about $50 \%$ of government revenues. This level of debt would not be sustainable over the long run. It was therefore hoped that with sufficient external support in the form of grants and concessional loans, and a financial contribution from the domestic financial sector, the ratio of debt to GDP could be brought down to a level that could be sustained in the long run.

16. The current government has embarked on a series of reforms since it took office in July 2005 . These included growth, privatization, public finance and debt management and financial and capital market reform measures. The growth-enhancing structural reforms have covered competition regulation, consumer protection, SME support, intellectual property rights and trade facilitation. The government has proceeded steadily with privatization, after appointing a Secretary General for the Higher Council for Privatization through a transparent and competitive process. Two mobile licenses were prepared for tendering with the help of two investment banks. The corporatization of Liban Telecom is currently underway. An IPP (Independent Power Producer) Law for the power sector was also ratified by parliament. Under public finance and debt management, the government established the Large Taxpayer's Office, and introduced new reforms for tax and public finance management. The government facilitated tax filing by mail and settlement through commercial banks. In addition, the government submitted more than fifty draft laws to parliament, among them: the Capital Market Reform Law, Tax Procedure Code, Treasury Single Account Law and Debt Management Office Law. Under financial and capital market reform, the government enacted a modern Securitization Law and a Fund Management Law, and is discussing other reform measures with the relevant parliamentary committees pending their ratification. 


\section{B. The Impact of the July Israeli War}

17. Attacks by land, sea and air resulted in tragic loss of human life, large-scale displacement and massive damage to private and public infrastructure, and a complete dislocation of the economy. The blockade that continued for a month after the cessation of hostilities further deepened the negative impact on the economy. By the time of cessation of hostilities, nearly 1,200 people had died as a direct result of the attacks, of which nearly one-third were children, and about 4,400 were injured. About a quarter of Lebanon's population was displaced during the war, and about 500,000 people saw their houses destroyed or damaged ${ }^{3}$. There were additional deaths and dozens of injured by unexploded ordinances (UXOs) after the war ended as a consequence of the estimated 1.2 million cluster bombs that were fired into Lebanon during the final days of hostilities ${ }^{4}$. Many professionals and entrepreneurs left the country, among them persons that had returned to Lebanon after the end of the 1975-1992 war, and about 30,000, many of them youth, lost employment.

18. The economic impact of the conflict is substantial in the short term and is likely to constrain Lebanon well beyond 2006 given the time it will take for the economy to recover from this significant setback. Based on assessments carried out by independent consultants retained by the Prime Minister's Office, the total direct cost of early recovery and reconstruction is now estimated at around US $\$ 2.8$ billion. The cost of reconstruction includes the cost of rebuilding and repairing private and public infrastructure, replacing lost assets, and compensating for private housing, which is by far the largest component of the losses.

19. The indirect impact of the war is much more significant. Real GDP growth, which was estimated at 5-6\% during the first half of 2006, (according to $\mathrm{INSEE}^{5}$, the growth during the first half of the year might have been even higher than $7 \%$ ), is likely to end the year at a negative $5 \%$. This represents a loss in output and income for 2006 in the order of US\$2.2 billion (at 2005 prices). The loss in output in the medium term (as compared to the levels that were envisaged) is a multiple of this amount.

20. The impact of the July war on the fiscal situation is severe. Due to the strong performance of the first half of 2006, the primary surplus was expected at $3 \%$ of GDP for 2006, but is now expected to shift to a deficit of $0.4 \%$ of GDP $^{6}$ (US $\$ 90$ million) after four consecutive years of surpluses. The overall fiscal deficit could reach $14 \%$ of GDP due to a revenue loss of around US\$180 million (the magnitude of the loss would have been much more severe had the US\$537 million of grants ${ }^{7}$ not been received), and to about US\$740 million increase in conflict-related expenditures (includes expenditures financed by grants that have been pledged). On this basis, total gross public debt is expected to reach US $\$ 40.5$ billion by the end of 2006 -equivalent to $180 \%$ of estimated post war 2006 GDP $^{8}$.

21. The increase in the government's financing needs as a result of the Israeli war comes at a time when the government is already facing very large debt obligations in the short term.

\footnotetext{
${ }^{3}$ The total number of housing units affected is estimated at more than 100,000 .

${ }^{4}$ Based on estimates of the UN's Mine Action Coordination Center (UN MACC), it is estimated that around one-third of the bomblets had not exploded upon impact.

${ }^{5}$ INSEE is providing technical assistance to the Lebanese government for national accounts statistics

${ }^{6}$ Note that this figure may differ slightly from the fiscal primary balance figure in paragraph 70 due to the two different methodologies for compounding debt service payments and foreign financed capital expenditures.

${ }^{7}$ Note that US\$537 million is the grant amount that is projected to be disbursed in 2006 only

"For further details, please refer to the "Impact of the July War on Public Finances in 2006" and to the" Impact of the July War on Public Finances in 2006, Updated Figures, Explanatory Note" published on the Ministry of Finance website www.finance.gov.lb.
} 


\begin{tabular}{|lrrrr|}
\hline \multicolumn{1}{|c|}{ Debt (as of June 30, 2006) maturing in the years } & $\mathbf{2 0 0 7 - 2 0 1 0}$ (US\$ billion) & \\
& $\underline{2007-08}$ & $\underline{2009-10}$ & $\underline{2007-10}$ \\
& 4.5 & 5.5 & 10.0 \\
Foreign Currency Debt & 11.4 & 3.4 & 14.8 \\
Local Currency Debt & 15.9 & 8.9 & 24.8 \\
Total Public Debt excluding interest & & & \\
(Principal payments except for discounted $t$-bills)
\end{tabular}

Indeed, $65 \%$ of the public debt outstanding prior to the July 2006 war matures during $2007-10$, in addition to most of the new borrowing during and subsequent to the July 2006 Israeli war in the remainder of 2006. Close to US\$16 billion of the debt outstanding as of mid-2006 matures in 2007 and 2008 alone. As a consequence of the war, and with the prospect of larger financing needs of the government, spreads on Lebanese foreign currency debt have risen. This will further aggravate the future fiscal situation as a direct consequence of the war.

\section{The Cost of the July Israeli War and International Support for Recovery \& Reconstruction}

22. The total direct cost to the Lebanese government of early recovery, reconstruction of public infrastructure, and housing compensations, that are to be covered by the Central Government budget is now estimated at about US $\$ 1.75$ billion. This excludes the private sector's and NGO's contribution to the reconstruction efforts, the compensation for destroyed private properties other than housing and the financing programs that aim for reviving private sector activity.

23. The international community reacted quickly and generously to support Lebanon. In addition to the deposits of US\$ 1 billion from Saudi Arabia and US\$500 million from Kuwait with BdL to help maintain confidence and monetary stability, the international community pledged generously to support reconstruction and relief efforts. On August 31, 2006, the Swedish government hosted a Conference for Lebanon's Early Recovery in Stockholm. At that Conference, Lebanon received indications of support amounting to about US\$900 million for humanitarian assistance needs and early recovery efforts. This financial support provided the conditions for the return of the quarter of the population that was displaced, and restoring minimum capacity in terms of infrastructure, access to basic social services and income generating activities, pending full reconstruction.

24. The pledges and commitments made prior to the Stockholm conference, especially by Arab countries, as well as those made on the occasion of the Stockholm Conference, including concessional loans for reconstruction, together with some reallocation of funds from existing project loans, are likely to cover the estimated total cost to the government of direct cost of early recovery and reconstruction, except for a remaining gap for the housing compensation component ${ }^{9}$.

25. For its part, the government established a number of mechanisms to ensure transparency and accountability with respect to the use of donor funds. These include a special account opened for each donor with the BdL specifying use of funds, and a UNDG Multi-Donor Trust Fund to be managed by UNDP to be used for those donors that have expressed a preference to channel their post-war assistance through UN channels, and allowing donors to sponsor a project directly (such as rebuilding

\footnotetext{
${ }^{9}$ The magnitude of this gap will depend on final estimates for housing compensation payments and the utilization of grants provided by Gulf Countries, in particular Saudi Arabia (US\$570 million), Kuwait (US\$315 million), Qatar (US\$300 million), Oman (US\$50 million), Iraq (US\$35 million) and UAE (in an amount to be determined). This excludes grants provided in kind, relief aid and humanitarian assistance, grants provided to NGOs and the private sector, grants for technical assistance and security, and donors' administration costs.
} 
bridges, schools, hospitals and villages). CDR will be responsible for funds allocated to reconstruction projects that it carries out, in compliance with donor requirements. In the case of housing, which is the largest component of the government's program, the government has opted for direct payments to home owners as they are best placed to procure construction and repair materials and services at the lowest possible cost, whereby checks are issued in favor of the beneficiaries as determined by the concerned government agencies and after a thorough audit conducted by an independent consulting engineering firm.

26. In order to improve aid coordination and harmonization, and the allocation of donor support to priority projects and programs, the government is putting in place a unified system of data collection and reporting. A Development Assistance Database is being established at the Prime Minister Office (PMO), with support from UNDP, while the Ministry of Finance (MoF) is establishing a transactional information system to monitor financial flows, with support from the World Bank. Both systems will be integrated and expanded to a unified system at MoF that will also cover external project loans and budget funds that finance the public investment program. MoF has established a donor coordination secretariat to support implementation and maintenance of the system.

27. In view of the substantial damage inflicted on the private sector (mainly agriculture and tourism), the government will provide some financial assistance as well as support through loan programs totaling up to US\$950 million, with a government subsidy on interest, and in some cases, guarantees. The government hopes to limit the cost of such subsidies with the help of lines of credits at concessional rates from international institutions such as the IFC, EIB, AFD, IDB, AFSED, OPIC and the OPEC Fund. These facilities are complemented by a number of temporary tax incentives to support business activity and promote investments as well as to help avoid large scale bankruptcies and business closures. In addition, the government will rely on commercial banks and existing microfinance institutions for lending to the private sector. The BdL, together with Kafalat ${ }^{10}$, would be responsible for overall supervision of the programs.

28. The direct outlays for recovery and reconstruction, while significant (US\$1.75 billion, excluding the reconstruction cost that is directly borne by bilateral donors and private sector), pale in comparison with the indirect costs in terms of the impact on the economy and on public finances. Even with the implementation of the government's reform program, including privatization and strong fiscal adjustment, fiscal deficits would, without external support, far exceed the fiscal deficits that were envisaged in the pre-war reform scenario. Not only has the public debt to GDP ratio risen, but Lebanon now also faces higher fiscal deficits. The cumulative incremental fiscal deficit for the period 2006-10 is estimated at US $\$ 5.9$ billion, inclusive of the cost of recovery and reconstruction ${ }^{11}$. The impact is mitigated somewhat by the pledged grants and loans for reconstruction, but incremental net financing needs of US\$4.5 billion remain for the period 2006-10.

\section{II. THE GOVERNMENT'S REFORM PROGRAM}

29. The pre-July war comprehensive economic reform program has been re-phased following the Israeli war. Apart from support to help Lebanon meet the direct cost of recovery and reconstruction, the need for external support to achieve a sustainable debt scenario has now risen further, and the near-term financing needs are substantial. The government remains committed to honor its debt obligations, as it has always done, even during the darkest days of war (in the midst of the war, in August 2006, the government paid the first principal installment due on a Paris II loan).

\footnotetext{
${ }^{10}$ A company owned jointly by the National Institute for the Guarantee of Deposits (75\%) and Lebanese commercial banks ( $25 \%$ ), audited by independent external auditors. It provides for loan guarantees and interest subsidies (financed by Government) for commercial banks lending to SMEs.

"The difference between the cumulative fiscal deficits in the pre-war reform scenario (2006-10) and the cumulative deficits for 2006-10 in the post-war reform scenario (see below).
} 
30. Even with a resumption of growth (reflecting the reconstruction impact and structural reform measures that the government will undertake), the post-war revenue base will be much smaller and the pre-war planned tax increases must now be delayed. Any early introduction of new tax measures would not yieid prospected results, and could well cause a slowdown in economic activity; leading to social and political unrest. On the expenditure side, following the Israeli war, it has become very difficult to reduce the ratio of current expenditures to GDP in the short term given the increase in security and social expenditures aimed at ensuring the delivery of basic public services in all regions and at compensating the war injured and families of deceased.

31. Similarly, unfavorable market conditions, and/or lower asset value, will delay the privatization plans (sale of assets) for some time, although the sale of the two mobile licenses should proceed shortly. Also, the preparatory process of restructuring and corporatization and establishing regulatory agencies and other sector institutions are moving forward.

32. On the other hand, social sector reforms and strengthening social safety nets become a critical element of a strategy aimed at ensuring the prevalence of the State over all the Lebanese territory, to the benefit of all denominational groups. A comprehensive approach to recovery and reconstruction is an equally important element of this strategy. Public sector reforms are key to improving the business climate, jumpstarting post-war recovery, and enhancing public sector performance to underpin the strategy of ensuring the State's presence and its efficient and transparent delivery of the recovery and reconstruction program.

33. In the absence of adjustment, and taking into account the impact of the July war, by 2010 the budget deficit would revert back to about $20 \%$ of GDP, debt service would absorb more than $85 \%$ of government revenues, and debt-to-GDP ratio would increase to about $215 \%$. The financing needs of the government would lead to higher interest rates, weaken the banking system due to increased exposure to sovereign risk, and ultimately threaten the stability of the exchange rate and prices, affecting mostly the poor and the middle class (mainly wage earners) who cannot protect themselves against these risks.

34. Lebanon has fallen short of achieving its full potential because of political tensions, complicated macroeconomic situation, and structural rigidities. Given Lebanon's significant human resources, well-educated workforce, large Diaspora, well developed banking sector, and attractive climate and geography, Lebanon should be able to achieve better economic performance and a higher standard of living.

35. The overriding objective of the reform program is to stimulate growth, create employment, reduce poverty, and maintain social and political stability. More specifically, the aim of the reform program is to raise the real growth rate to at least $4-5 \%$ over the next five years, improve social indicators, and reduce regional inequalities. Since the main source of economic vulnerability in Lebanon is related to the large public debt, placing this debt on a downward trajectory towards sustainability - while protecting the most vulnerable segments of the population-is a prerequisite for achieving the objectives of the program.

36. The reform program is an integrated package that will succeed only if its major components are implemented. In particular, external support is an integral part of the program and is crucial for the success of the reform efforts. It is articulated around six pillars including the financial support of the international community: 
- Growth-enhancing reforms encompassing a large number of measures and laws that would increase productivity and reduce cost, which would enhance the competitiveness of the Lebanese economy;

- A social sector reform agenda to improve social indicators and strengthen (develop) social safety nets to protect the most vulnerable segments of the population;

- A strong phased fiscal adjustment that aims at increasing the primary surplus through streamlining expenditures-including by reducing waste (including legalized waste) and reforming state owned enterprises (SOEs) more specifically Electricité du Liban (EdL) — and raising revenues in ways that minimize the negative impact on the poor;

- A privatization program directed primarily at increasing investment, reducing the stock of public debt, and spurring economic growth;

- A prudent monetary and exchange rate policy aimed at maintaining price stability (and with it social stability), facilitating credit to the private sector, and maintaining a sound banking system;

- International financial assistance to help Lebanon finance the direct and indirect cost of the July war as well as to complement the domestic adjustment efforts, primarily by reducing interest payments on public debt and creating the kind of confidence that would encourage private sector investment and ease the pain of a domestic adjustment after the war.

\section{A. Growth-Enhancing Struetural Reforms}

37. Enhancing growth is the corner stone of a successful reform program and a precondition for creating employment, improving living standards, and reducing the public debt burden. Economic growth is multifaceted, however, and there is no single measure or a set of measures that would boost growth overnight. While ensuring macroeconomic stability and accelerating public investment (discussed below) are all important for growth, other elements of the structural reform and growthenhancing agenda encompass a large number of measures, such as governance (including aspects related to corruption), the business environment, trade liberalization, capital markets development, and debt management.

\section{Governance}

38. Good governance is a prerequisite for investment and growth. Acknowledging that governance should begin with the public service, the Office of the Minister of State for Administrative Reform (OMSAR) has prepared an administrative reform strategy that focuses on the core functions of a modern state. The strategy articulates several objectives that cut across all parts of government and aim at creating a transparent, accountable and effective public administration. These objectives aim to: (i) develop and establish modern management capacity in key administrations such as the central control and oversight agencies; (ii) reduce the size and cost of the public administration through streamlining its overall organizational structure and functions; (iii) modernize legislation especially that related to public procurement and accounting; and (iv) promote a citizen-oriented administration through simplification of procedures and advancing e-Government. Many of these measures have started a while ago and will continue over the medium term.

39. In view of its stated objective of greater accountability and better governance, the government approved and sent to parliament a draft law aimed at hiring an international and reputable firm(s) to audit public finances and public enterprises over the last fifteen years (1990-2005). It is expected that the selection of the firm(s) will take place after the adoption of this law by parliament and work will begin soon after. Since the risk of corruption is perceived to be prevalent mainly in large infrastructure projects and public procurement more generally, the government intends to expedite the completion of the new procurement law with the related decrees and implementation tools to be effectively deployed by mid-2007. This Jaw will provide for a more efficient use of public funds while enhancing transparency in accordance with international business standards-the present procurement 
law dates back to the early 1960 s. On a parallel and related track, establishing a regulatory body for maintaining, monitoring and updating the public procurement system will also be on the agenda. In addition, the role of the Court of Accounts as an ex-post agency will be revived to ensure that all budgetary spending is in accordance with budgetary allocations. Fighting corruption also requires empowering state watchdog institutions to take punitive actions and enforcing conflict-of-interest regulations against senior public officials.

40. The government intends to address governance issues at the agency level in a pragmatic way. Given the difficulties in resolving these issues all at once, a few pilot agencies will be selected for close scrutiny. A successful reform in one public agency with early tangible results would strengthen the constituency for reform, and provide useful levers to sway public and official opinion. In this regard, the government has decided to start with two important ministries, the ministry of public works and the ministry of finance. Two internal control units will be established to monitor the operations of the various departments in the two ministries to ensure that they follow best practice in service delivery and to deal with corruption incidents. These units should be operational by end-2007. This pilot program could be extended to other public entities in 2008-10.

41. More generally, the government will continue to streamline and automate work procedures to reduce the citizen interface with public sector employees and reduce the risk of corruption. Several initiatives have already been taken in this regard at the level of the ministry of finance where information and procedures have been automated for some departments such as the cadastre, customs and taxation. The reform of budget preparation and implementation, and the elimination of all nuisance taxes will help fight corruption. These initiatives will be extended to other ministries and public agencies over the next few years.

42. The recent decision by the government to have transparent, merit-based, and proper procedures for public sector recruitment is a very important step in isolating recruitment from political and confessional considerations. While the equal confessional distribution of top public sector positions, as determined by the constitution, cannot be changed at this time, every effort will be made to hire the most qualified and to do away with the practice of assigning specific positions to specific confessions as long as the power sharing arrangement is respected. In this regard, the role of the Civil Service Board will be enhanced and proper and transparent assessment mechanisms will be put in place to improve selection.

\section{Financial Sector Reform and Debt Management}

43. The government plans to address the long-standing issue of underdeveloped capital markets as part of a broader strategy to modernize the non-bank financial sector and stimulate investment and growth. Apart from its growth-enhancing effects, capital markets development would help diversify risk and increase financial resilience in the face of shocks. The role of the stock market is also instrumental in the coming period as it will facilitate the corporatization and privatization drive, help attract long-term financing mainly from the region--especially at times of high oil prices-and provide the private sector with working capital. Similarly, the development of a secondary market for fixed-income instruments should contribute to financial deepening.

44. Several measures have already been taken to enhance the functioning of Beirut Stock Exchange (BSE), and in December 2005, parliament approved two laws on securitization of assets and on fund management, and is currently discussing the draft law on dematerialization of securities, the securities lending draft law, the insider trading draft law and the Capital Markets draft law (see Box below). The principal aim of the Capital Markets draft law is to provide the framework for the regulation and development of the capital markets in Lebanon, and hence build confidence in the quality of the market for both local and foreign investors. An important feature of the law is the 
establishment of the Capital Markets Council, a new independent regulatory body responsible for overseeing regulating and developing Lebanon's capital markets. In addition, an advisory team set up by the ministry of finance has come up with several recommendations that will further develop the capital markets.

\section{Box 1. Capital Markets Reform Measures}

A. The following measures have already been taken:

- Allowed remote trading and listed government Eurobonds on Beirut Stock Exchange.

- Offered a tax incentive for companies listing on the BSE, by reducing the dividend tax from $10 \%$ to $5 \%$

- Eliminated commissions paid on Eurobonds transactions listed on the BSE carried out on the official or secondary markets.

- Adopted the Asset Securitization Law and the Fund Management Law by parliament in Dec. 2005.

B. The following measures will be taken next year to further strengthen the capital markets:

- Pass the following draft laws: The dematerialization of securities law, the Securities Lending Law, the Insider Trading Law and the Capital Markets Law-all of them are currently under discussion at the Parliament.

- Set up the Capital Markets Commission, including a specialized financial court.

- Develop secondary market liquidity by introducing longer maturity benchmark instruments, and establishing primary dealers in LL issues.

- Establish a Delivery Versus Payment system for the LL, and increase trading hours and reduce settlement trade to $T+2$

- Develop an official stock market index

- Broaden investor base and attract institutional investors such as insurance companies and the National Deposit Insurance Company (NDIC)

45. In view of the high level of debt, improving debt management could have a positive fiscal impact. A draft law to establish a modernized debt management office at the ministry of finance has been reviewed by committees in the parliament. The debt office will assume the responsibility of the operational aspect of the debt in both local and foreign currency. In addition, the government is working towards developing a formal debt management strategy and improving cash management. In parallel, the government is also looking into the issue of strengthening domestic debt market and introducing primary dealers system that would enhance liquidity in the secondary market.

46. The draft law recommends the creation of "Higher Council for Debt Management" under the chairmanship of the minister of finance and comprising representatives of $\mathrm{BdL}$ and of various departments dealing with debt issues at the ministry of finance. In addition, the ministry of finance and the central bank are coordinating their efforts to improve cash management which would have a significant impact on debt management.

\section{Improving the business environment}

47. The government is committed to undertake a whole range of reforms with respect to business laws and regulations, some of which have been under consideration. The key objective of these legal and regulatory reforms is to improve competitiveness and reduce the cost of doing business in Lebanon. A number of these laws fulfill the government's commitments as part of its membership in the World Trade Organization, which is expected by the end of 2007. Other initiatives are part of the Action Plan that the government has agreed on with the EU as part of the European Neighborhood Policy. In addition, the government's support and reform programs will have a special focus on SMEs, given their potential for contributing to employment creation. 
48. Various business surveys point to the high cost of doing business in Lebanon compared to regional and international standards. This cost is normally based on criteria such as (i) minimum capital requirement and cost of registration; (ii) the time it takes to get a license, start a business or close a business; (iii) the flexibility of the labor market and the ease of entry and exit of workers; (iv) the complexity of the tax system; and (v) the enforcement of contacts. The government plans to address these issues by designing a well-sequenced action plan for the next five years (Box 2).

49. Several other measures are under consideration to ameliorate the business climate. They include (i) ratifying a modern competition law and removing state protection including exclusive agency rights, which would reduce monopoly power and prices by 2007; (ii) adopting an e-commerce law and establishing business development centers (incubators) to incubate new companies; and (iii) ratifying the insurance draft law to regulate the sector, galvanize the stock market and attract new investors.

\section{Box 2: Improving the Business Environment}

The government intends to improve the business environment and reduce the cost of doing business in Lebanon through:

- Lowering the minimum capital requirements and the cost of registration by mid-2007.

- Reducing the time it takes to obtain a business license, and the cost of opening and closing a business, which is high in Lebanon by regional standards by end-2008.

- Improving access to credit. The system of subsidized interest for productive investment, a scheme which is supposed to be managed by the central bank, should be reviewed with a view to improve its efficiency.

- Strengthening the role of Kafalat (which provides loan guarantees for worthy investment) and promoting the creation of venture capital funds should also facilitate financing. Reforms in the fiscal and capital market areas will reduce the need for government to borrow from banks, and create a variety of financial products that should facilitate access to credit.

- Simplifying further the tax procedures and reducing the number of separate taxes and fees. While Lebanon ranks well in this category relative to the region, the ongoing tax reform efforts including the tax procedures code and the elimination of the nuisance taxes will help (2007).

- Expediting the clearance of imports, including by increasing automation at the port, and lowering the related cost. A one-stop-shop at the Port of Beirut will provide better services to trade and export.

- Modernizing the existing labor laws and reforming the end of service indemnity system as part of the pension reform (outlined below).

- Strengthening and shortening the enforcement of legal contracts by increasing the number of judges and court offices and the number of court rooms, improving court staff training, and opening small claim courts with no right for appeal (2008-10).

50. To encourage the recovery of the private sector in the aftermath of the Israeli war, the government is undertaking a series of specific measures and incentives at the tax, regulatory, financing, and promotional levels. The government has laid out a comprehensive plan for recovery of the private sector that is based on three main pillars: (a) a basket of tax incentives developed by the ministry of finance to support private sector enterprises and reduce the burden of the war; (b) programs that allow easy and low cost access to financing on flexible terms/long maturities, as well as allowing for restructuring of outstanding loans in addition to special direct arrangements between banks and indebted clients; and (c) measures aimed at promoting Lebanese exports and promoting Lebanon as a regional business center.

51. Improving competitiveness of the Lebanese economy more generally is critical for promoting exports, creating employment, and enhancing growth. The cost of production in Lebanon is relatively high, affecting the ability of Lebanese products to penetrate regional and international markets. In addition to the need to eliminate barriers in the face of increasing openness and liberalization, there is a need to reduce the cost of production resulting mainly from unreliable supply of electricity, the high 
cost of telecommunication, as well as cobweb administrative procedures that put an undue pressure on costs and affect competitiveness (all of which are integral part of the reform program). To monitor competitiveness indicators on a continuous basis, the government will establish a Competitiveness Council. This council would be composed of major public/private institutions to report on, and deal with, business activities bottlenecks and act as "bureaucratic Inertia Buster".

\section{B. Social Sector Reforms}

52. The economic reform program would be incomplete if not accompanied by a comprehensive social reform program. As a result, the government has developed a social action plan as an integral part of its fiscal and economic reform program. Social sector reform is beneficial for promoting sustainable and equitable development and combating poverty, and hence achieving the government's commitment to the "Millennium Development Goals". Such reform is crucial for long-term sustainable economic growth. The main objectives of the social action plan are to: (i) alleviate poverty and improve the quality of education and health indicators; (ii) improve the efficiency of public social spending and keep it at an appropriate and sustainable level; and (iii) reduce regional disparities in development indicators through a proper distribution of investment and other resources and encourage investment and other job-creating activities in the more deprived areas.

53. Social indicators are not commensurate with the level of spending, which is comparable to that in developed countries. In 2004, social spending (public and private) amounted to $21 \%$ of GDP (excluding pension). In 2005, public spending on social services accounted for approximately $42 \%$ of primary expenditures, $27 \%$ of total expenditure and $8 \%$ of GDP. Despite this relatively high social spending (on health and education), indicators remain unsatisfactory. In the education sector, the drop out and repetition rates are relatively high, hovering around $22 \%$ and $23 \%$, respectively. In the health sector, infant and maternal mortality were 18.6 per thousand and 88 per one hundred thousand (Papfam, 2004), respectively.

54. The incidence of poverty in Lebanon might appear limited and controllable, since although $25 \%$ of the population lives in relative hardship, only $4 \%$ live in extreme hardship (Living Conditions Index, 2004). However, there are large regional disparities in poverty incidence and intensity in the country. Available statistics show that peripheral regions suffer from high rates of extreme poverty (reaching $11 \%$ in North and South Lebanon, compared to $1 \%$ in Beirut and some regions of Mount Lebanon). Data also reveal that densely populated urban areas, such as the Northern and Southern Suburbs of Beirut, Tripoli, Saida and Baalbeck, have the largest concentrations of the poor.

55. Living conditions improved considerably between 1995 and 2004 (Comparative Living Conditions Index), with a decrease in the percentage of the deprived population from $34 \%$ in 1995 to $24.5 \%$ in 2004 . This progress is attributable to improvements in the social.

56. The government is committed to improving both the efficiency and the targeting of its social sector expenditures and had already started to address this issue in a comprehensive manner prior to the July 2006 war. Steps were initiated to reinforce the role of the Ministry of Social Affairs (MoSA) in social policy making and development. The war has slightly delayed the implementation of the reform agenda and has significantly exacerbated income-poverty, increased social needs, and expanded the incidence of poverty and vulnerability to other groups. Besides the loss of life and human capital (injuries, disabilities, adverse schooling effects and emigration), increased unemployment has contributed to an increase in poverty and vulnerability. Although the Israeli war has affected all regions and all population groups, the poor and vulnerable remain more susceptible and less capable of coping with the adverse impact of the war. 
57. In areas directly affected by the hostilities, recovery efforts will improve the capacity to cope with the losses in the short term but remain insufficient to create sustainable growth; whereas in other historically poor areas that were not directly affected by the war (such as Akkar), the situation may worsen as a result of chronic poverty, increasing marginalization, and decreased access to employment if not covered by the social program. Other regions that were neither directly affected by the hostilities nor included high poverty rates, such as Greater Beirut (excluding the Southern Suburbs) and Mount Lebanon, where $57 \%$ of the labor force and $54 \%$ of enterprises are concentrated, will be also affected from the structural impact of the war due to loss of jobs and investments. Even within each region, the adverse direct and indirect effects of the war may be unevenly distributed.

\section{Main elements of the social plan}

58. Prior to the July 2006 war, the government had started the implementation of a social action plan aimed at strengthening existing programs and developing new programs to address the abovementioned social indicators. Immediately after the end of the hostilities, the international community has provided support and direct assistance to help deal with the aftermath of the war and the government intends to provide fiscally-neutral incremental financing to improve social conditions through well-targeted interventions to address the needs of the most vulnerable groups.

59. The government has established an inter-ministerial committee to coordinate the efforts of various ministries and prepare a comprehensive medium-term social development strategy. Such a strategy is a first step towards improving the efficiency of social spending and reducing waste, which are caused by the lack of coordination between concerned ministries. In addition, each of the concerned social ministries will develop its own sectoral strategy.

\section{Box 3: Some Elements of the Medium-Term Social Strategy}

- Reactivating the inter-ministerial committee for social policy that comprises the Ministries of Health, Education, Social Affairs, Labor, Economy, and Finance. The role of this Committee is to set overall sector strategies and priorities, and monitor and evaluate the implementation of social strategies. The Committee will be supported by a technical secretariat.

- Reducing overlapping in the provision of social services across ministries, as well as unifying social funds under one transparent and efficient administration. This measure could be implemented in 2007.

- Processing and analyzing the results of the 2004 multi-purpose household survey, and designing targeting mechanisms that would help improve the coverage, integration and impact of the existing, but fragmented social safety net.

- Completing a poverty assessment of the country, and introducing appropriate social safety net measures accordingly.

- Expanding basic health services coverage and improving efficiency and quality; reducing infant and maternal mortality including by enhancing preventive health care and increasing the vaccination rate for poor children in selected areas.

60. In order to enhance the efficiency of public social spending, the government will also improve targeting mechanisms and eligibility criteria. In the same vein, the results of the Multi-Purpose Survey will be used for evidence-based policy making and programming. Within the broader context of a statistical data improvement, the implementation of the Statistical Master Plan (SMP) will be accelerated. The SMP would set out a medium-term action plan for producing and disseminating data, building statistical capacity and reforming concerned institutions. 
61. In education, many schools, especially in peripheral areas, lacked adequate equipment, with coverage and quality problems. In addition, during the July 2006 war, more than $15 \%$ of public schools sustained serious damage and large numbers of teachers and students were displaced. The recovery of the sector is well underway and most of the capital costs needed for rehabilitation were pledged by donors. Concurrently, the government will accelerate the implementation of comprehensive sector reform program that will contribute to efficiency gains and improved equity. Also, gradual measures to rationalize sector expenditure will be implemented following the completion of school mapping and a teacher rationalization study. These reforms will be underpinned by strategic planning and decision support tools to be developed in the course of 2007 which will help achieve the said objectives. This includes strengthening of education policy making and planning capability at the Ministry of Education, the installation of a sector management information system to monitor.key sector outcomes, and the development of evaluation tools for the instructor's development program. Within the sector, addressing the issue of the high level of dropouts in certain regions, which was further exacerbated by the war, will be a sector policy priority.

62. In the health sector, apart from physical damage (16 hospitals and 65 outpatient facilities were damaged), the war has considerably increased sector expenditures. Government subsidies to health insurance funds and reimbursements to private hospitals will increase on account of treatment of the injured and disabled. This underscores the urgency of implementing key sector reforms. The government will take a number of measures aimed at enhancing its capacity to improve the overall public health system and narrow the equity gap in terms of sector outcomes. These include: (i) reforming health insurance to ensure greater efficiency and improved access (e.g. harmonizing or integrating public health insurance funds, and strengthening management and contracting capacity of public funds); (ii) developing targeting methods to better evaluate the coverage provided by the Ministry of Health; (iii) upgrading and institutionalization of the national hospital accreditation program and its expansion to primary health care; (iv) introducing performance-based contracting for targeted primary health care services, particularly in poorer areas; and (v) enacting "Carte Sanitaire" legislation to better monitor and regulate health care investments.

\section{Specific social interventions}

63. The government intends to reduce poverty by improving a number of existing programs and introducing new programs within the framework of the Ministry of Social Affairs. The most important programs are cash transfers to poor senior citizens, female-headed poor households, and disabled poor. Beneficiaries of these programs will be identified based on transparent eligibility criteria established according to poverty targeting maps.

64. The government will also work on reducing regional disparities through promoting local development as an efficient tool for balanced regional development and poverty reduction. This includes increasing capacities of the Social Development Centers of MoSA and creating coordination and synergy among key local development actors (e.g. MoSA, Economic and Social Fund for Development, Community Development Programme, UNDP, international donors and organizations, and active NGOs).

65. The government will develop programs to improve education indicators, particularly to reduce student drop-out and repetition rates in public schools in poor regions. These programs aim at reducing the burden of the education cost on the poor households in targeted areas through subsidies to school related expenses (books, stationery, transportation and school feeding), on condition that these students remain in school until the end of the compulsory educational stage (age 14). The Ministry of Education will also establish remediation centers to help poor students and provide technical training in various specialized disciplines. Poor households will be also exempted, either partially or fully, of public school registration fees. 
66. The government is also committed to improving health indicators. The Ministry of Public Health will introduce a number of health programs, such as increasing the number of vouchers provided to poor pregnant women for clinic visits before and after giving birth. In addition, needy patients will be totally exempted from hospitalization fees and a voucher system will be established for poor patients with chronic diseases. The ministry of public health will also cooperate with the ministry of education to enhance the school health program, by raising the number of MD visits to schools and expanding the program to basic education.

\section{Pension Reform}

67. The reform of the pension system in Lebanon is a priority because of its social, economic, and fiscal impact. The social aspect of pension reform relates to equity considerations and to the need to protect the poor or those who do not have the means to save sufficiently for retirement. The fiscal cost of the pension schemes in Lebanon is very high by regional and international standards and is likely to increase sharply over the medium and long term if not addressed soon. The private sector end-ofservice indemnity scheme does not provide adequate income protection during old age yet imposes a heavy burden on employers and does not facilitate switching between jobs. As a result, it reduces the incentive for hiring and introduces more rigidity in the labor market with negative impact on employment and growth.

68. The two public sector defined benefit schemes, the civil and military systems, impose a heavy burden on the budget (2.5\% of GDP in 2004) with an implicit debt of close to $60 \%$ of GDP (defined as the present value of the pension promises for current retirees and current contributors). The private sector system, which is managed by the National Social Security Fund (NSSF) while financially viable for another 15 years or so, could have major contingent liabilities on the budget.

69. With technical assistance from the World Bank, the government will overhaul the pension system and to this end it is preparing a draft law to be submitted to parliament. The objective of the reform is to integrate the three systems into one modern fully-funded defined contribution (FF-DC) scheme by 2008 while preserving their acquired rights under the present system. However in the case of the civil service and the military, the government will face transition costs over the short term. These costs will be estimated and appropriate financing mechanisms devised. Over the medium and long term, however, the reform is expected to bring considerable savings to the government and future generations. The main features of the pension reform are:

- The undertaken reforms will ensure the continuation of all allowances and indemnities to existing members.

- The closing down of the systems for civil servants and the military to new entrants with new civil servants and military personnel joining the new FF-DC system. Current contributors can choose to move to the new system on a voluntary basis.

- The pension scheme for members of parliament will be revised to make it consistent with the rest of the public sector.

- The new pension will extend the coverage to self-employed and casual workers with limited saving capacity. 


\section{Fiscal Adjustment and Structural Reform}

70. Fiscal adjustment is essential to put debt on a downward trend. The government will undertake strong and sustained fiscal adjustment efforts beginning in 2007 (implementation of some reform measures is expected during 2007) to gradually improve the primary balance, from a deficit of nearly $1 \%$ of GDP in 2006 to a surplus of $8 \%$ of GDP in 2010 so as to reverse the debt dynamics and place the debt-to-GDP ratio on a downward path. Fiscal adjustment has to be shared equitably to be sustained and every effort will be made to minimize the impact of fiscal adjustment on the poorer segment of the population. It will require both strong expenditure and revenue measures and an improvement in the budget preparation.

\section{Expenditure Measures}

71. The government has generally followed a tight expenditure policy in recent years and will continue to rationalize current spending. Current expenditures, excluding interest payments, at $17.8 \%$ of GDP (in 2005) are not high by international standards and thus there is not much room for cutting them further beyond the pressing need to reduce waste and increase effectiveness and productivity of spending. The government fully recognizes that improving public management is crucial to enhance efficiency of and reduce waste in public expenditures. Addressing these issues upfront is important to improve the business environment and foster growth. The key areas for expenditure reform include rationalizing non-interest expenditures, containing the wage bill, and reforming public sector enterprises, especially EdL ${ }^{12}$.

\section{Primary expenditures}

72. The government intends to rationalize current expenditures through various measures and reforms including (i) reviewing the salary and benefit structure in some public entities in particular, and the salaries of current and previous members of parliament as well as ministers, in addition to allocations to the Presidency of the Republic and the Presidency of the Council of Ministers; (ii) reducing to a minimum official travel expenses and revisiting travel allocations; (iii) saving on transportation allowances through extending the working hours in the public sector from the $\mathbf{3 2}$ hours a week to at least 36 hours by mid-2007; (iv) reducing waste related to gasoline consumption and communication costs in the public sector; and (v) closing the Fund for the Displaced and the Council of the South-which are expected to fulfill their intended mission by 2008 . While the financial impact of these measures could be limited to about $0.2 \%$ of GDP, their impact on the overall efficiency of government operations should not be underestimated.

73. The government will contain the wage bill in the medium term through limited hiring and productivity increase and through automation and promotion of e-government, allowing the size of the public sector to shrink by attrition. However, with respect to military and security staff, there will be an increase in the short run to meet security needs and to honor Lebanon's obligations in the context of the UN resolution 1701. Over the next five years, $20 \%$ of public sector employees would retire (and $45 \%$ in the next 10 years) providing a good opportunity to rejuvenate the public sector while at the same time reducing its size and enhancing its efficiency without having to force any employee out. Replacing only partially those who are retiring in the next five years would lead to important savings. It is expected that the wage bill to GDP would be reduced by $2.0 \%$ by 2011 .

74. In this context, overstaffing in various ministries and in some public entities that are no longer operational will be examined closely. Overstaffing exists mainly in the ministry of education since public education in Lebanon has one of the lowest teacher-to-student ratios in the world (one teacher

\footnotetext{
${ }^{12}$ Transfers to EdL will gradually decrease from a level of $3.5 \%$ of GDP in 2006 to $0.3 \%$ of GDP in 2011 , based on the Power Reform Program and World Bank October 2006 oil price forecasts.
} 
per 9 students). It is worth noting, though that some schools suffer from severe shortages while others have an abundance of teachers, reflecting a mal-distribution of schools among regions. Excess employees in the public sector in general will be identified (2006-2008) through a civil servant census, and will be put under the Civil Service Board with possible reallocation to other ministries after undergoing proper training to improve their capabilities.

75. As regards public investment (capital expenditures), other than recovery and reconstruction outlays, Government plans to maintain the same level of capital expenditures to GDP, while increasing the share of foreign financed investments. In parallel, cost recovery will improve, as a result of restructuring and privatization in a number of sectors. For example, government subsidies to EdL are expected to drop as a result of the power sector reform plan

76. The level of the July war-related capital expenditures needs to be increased to account for the reconstruction process. In tandem, the efficiency of capital expenditures needs to be improved, to support the government's objectives of growth, enhance social conditions, and minimize regional disparities.

\section{Public Investment Program}

77. The government intends to have an adequate level of capital expenditures to maintain existing infrastructures and prevent its rapid deterioration and to invest in much needed new investment projects. The government aims to keep capital expenditures, excluding war-related spending, in the medium term at about $2.7 \%$ of GDP. However, the share of foreign-financed investments should be increased to an average of $45 \%$ during the period 2009-2011 (from the current 38 percent share). In addition, as part of the government's overall strategy, the private sector will play a greater role in infrastructure investments and in the provision of public services through various arrangements and in partnership with the public sector. Whereas the public investment program during the 1990s focused heavily on rebuilding the country's physical infrastructure, in particular transport, power and telecommunications, the future program, excluding the reconstruction program resulting from the July/August 2006 war, will put greater emphasis on providing basic public services.

78. The total loans that are likely to be used over the next three years are estimated at about US $\$ 880$ million out of US $\$ 2.12$ billion representing the total amount of undisbursed concessional loans. These loans stand for an average of $80 \%$ of the cost of the projects to which they are allocated, whereas the remaining part is to be financed by the treasury. The available loans will be allocated to several projects related to public services and infrastructure, as well as to socio-economic and productive sector projects covering all Lebanese regions. These projects are at different stages of implementation: some are being implemented but not yet completed, while others are still at the beginning of the implementation process.

79. A host of factors have played a role in the low level of utilization of foreign loans in recent years. Implementation difficulties and fiscal constraints were exacerbated by the unsettled political environment. Expropriation delays and shortages of local counterpart funds slowed down project implementation. Moreover, delays in parliamentary ratification of signed loans contributed to delays in project start-ups. As the generic and systemic problems are now being addressed, the rate of project implementation will improve from recent levels. In particular, the government will also ensure that within the overall fiscal constraints there will be timely and adequate availability of local budget funds to projects that are underway so that their benefits can be reaped as soon as possible. 
80. Implementation has also suffered from delays due to the low capacity in line ministries, and the resulting overload on CDR. However, to restructure CDR's capacity to undertake reconstruction and development capital investments, the government has embarked on a series of measures that will address CDR's effectiveness. These include strengthening the capacity of CDR's procurement, financial management and legal functions, and streamlining related internal processes from bid and terms of reference preparation to contract management and disbursement.

81. The need for public investment in physical infrastructure will decline after completion of the reconstruction, and with the privatization envisaged in the government's reform program. The government will seek a greater private sector role in sectors such as telecommunications, power, roads and urban development in the context of public-private partnerships. Within the overall financial constraints of the macroeconomic program, the focus of the public investment program will increasingly be on sectors where remaining needs are significant, such as water and wastewater, the environment and rural development.

82. The government is currently reviewing the entire program of ongoing projects and programs, with a view to deciding whether, given the reconstruction needs, implementation constraints and current priorities, the scope of ongoing projects and programs should be reduced, projects that have barely started should be continued, and certain loan agreements which have been signed should be either cancelled or renegotiated. The government will consult with lenders and donors on the steps to be taken as a result of this review and, in case of cancellations, lenders and donors will be asked to consider reallocating funds within the same sector for activities that have a higher priority (including to cover recovery and reconstruction expenditures that have no alternative funding), or provide new financing for high priority new projects within the medium term capital expenditures program.

83. The scope for initiating new projects with budget funds will be limited, given the fiscal and debt constraints. New, generally small, investments that ensure that benefits from past investments are fully realized will have a high priority. For most new projects, the government will seek foreign project loans or, wherever feasible, partnerships with the private sector. The selection of new projects and programs will take into account the need for an equitable regional balance, as well as the need to fulfill basic needs and reduce poverty. In this regard, the government will place top priority to selecting those projects that generate economic activity and new jobs in the outlying regions. Upon completion of this review, the government will, by the end of the first quarter of 2007, adopt a detailed public investment program for the medium term that is consistent with the overall macroeconomic targets and current priorities.

\section{Other fiscal structural measures}

84. In order to enhance the transparency and efficiency of public spending, the government intends to implement a number of measures to improve expenditure management. These measures should be implemented by 2008 and include (i) expanding the coverage of the budget to include the foreign-financed public investment executed by CDR (ii) preparing a medium-term expenditure framework and adopting top-down approach in budget preparation; (iii) eliminating the budget carryovers on current expenditures and containing treasury advances; and (iv) implementing the Treasury Single Account to improve cash management. The ultimate objective of this reform would be to develop a modern Budget System Law (BSL), which will be the anchor for all the reform measures. 
85. The government will look into the possibility of adopting a fiscal accountability law that would set targets for some important macroeconomic variables, particularly the level of budget deficit or public debt. Constraining the ability of the government to borrow, for example, would force it to undertake the necessary reform measures instead of resorting to more indebtedness. Such a law would oblige future governments and parliaments to adhere to the set targets and ensure fiscal discipline in the future.

\section{Reform of the Power Sector}

86. The reform of the power sector is the most important expenditure measure. Also, electricity being a major input element to the local economy, improving service quality would contribute to economic growth in Lebanon. Lowering the budgetary support to EdL would pave the way for achieving fiscal sustainability, stimulating economic activity, and reducing the need to raise the tax burden in the future.

87. EdL has been beset by major governance problems, resulting in significant technical and nontechnical losses (estimated at $15 \%$ and $20 \%$, respectively) and about $6 \%$ loss on collection-a total loss of more than $40 \%$ of the power generated. With fuel prices high, and in the absence of alternative fuel sources or bold measures this year, budgetary support to EdL reached about US\$1 billion in 2006 , or about $20 \%$ of fiscal revenues and $3.5 \%$ of GDP, a real hemorrhage for public finances. Overall, total outlays towards the power sector including the service of the debt incurred amount to more than a quarter of the total public debt. Reducing losses and strengthening sector capacity to encourage private sector involvement will be achieved through a set of short and medium-term actions. The government recognizes that there are no quick fixes for EdL, but savings of about $3 \%$ of GDP could be realized over the next five years based on various measures (Box 4) while awaiting EdL privatization (See below).

\section{Box 4: Reform of the Power Sector}

Reforming EdL requires a number of measures at different levels:

Under enabling initiatives, the government will:

- Appoint qualified advisors to the Ministry of Energy and Water, EdL and HCP

- Complete the auditing of EdL financial statements for 2001 through 2006

- Appoint a new Board of Directors for EdL

- Establish the Electricity Regulatory Authority and design its bylaws

- Introduce potentially necessary amendments to Electricity Law $\mathbf{4 6 2}$

- Corporatize EdL

- Design establishment decree and new bylaws for EdL

- Unbundle generation, transmission and distribution functions of EdL

- Complete the establishment of a National Control Center to audit volumes and performance (contract already signed).

Under short term restructuring initiatives, the government will:

- Modify restrictive oil specifications based on 2003 study

- Negotiate additional bilateral contracts for fuel oil and gas oil to reduce high premiums (Ongoing)

- License private companies to install and operate remote meters

- Reduce illegal network connections and enforce bill collection through support from security forces and the justice department (Ongoing)

- Secure the supply of liquefied natural gas to the Zahrani plant 
- Secure the supply of natural gas to the Deir Ammar plant

- Build a gas pipeline between the Zahrani and Baddawi plants

- Complete the expansion and rehabilitation of the $220 \mathrm{KV}$ transmission network

- Complete the infrastructure needed for the Ksara Station (including $400 \mathrm{KV}$ network) to allow Lebanon's

integration into the regional electrical network known as the "Seventh Joint"

- Decide on the viability of the Zouk and Jiyeh plants

- Privatize EdL

Other potential improvements would cover:

- Rehabilitate existing power plants, particularly Zouk and Jiyeh

- Install additional capacity in Deir Ammar and Zahrani (with private sector participation)

- Sign contracts with private sector companies to produce electricity (outside main urban centers) and sell to EDL (inside main urban centers)

- Increase hydro-electrical production capacity by issuing BOT contracts

- Provide the necessary investments to improve transmission

- Provide the necessary investments to improve distribution

-Secure more financing to invest in additional $2300 \mathrm{MW}$ to be ready by 2015 to meet the increasing demand for energy (add $1000 \mathrm{MW}$ to replace retired existing capacity and $1300 \mathrm{new}$ additional capacity)

88. To ensure the success of the power reform program, consultants will be engaged in early 2007 with international donor support to: (i) assist the Ministry of Energy and Water (MEW) in designing, implementing and monitoring and evaluating policy reforms of the power sector; (ii) assist EdL in its day to day operation of the entity with a focus on technical and financial aspects; and (iii) assist HCP in the restructuring and privatization of EdL.

89. As part of the program aimed at reducing non-technical distribution losses, the government is preparing tenders for the provision, installation and management of 1.2 million remote-controlled power meters which will help identify theft and non-payment. In addition, ongoing transmission investments, including a national control center will contribute to technical loss reduction. With respect to generation, an operation and maintenance contract is in place for the two main power plants. In order to increase generation efficiency and reduce costs, Government aims to substitute fuel with gas by: (i) providing Liquefied Natural Gas (LNG) to the Zahrani plant; (ii) providing natural gas through a regional pipeline to the Baddawi plant; and (iii) constructing a gas pipeline between the two power plants. With regards to the expansion of future generation, the government intends to rely on the private sector under Independent Power Producer arrangements.

\section{Revenue Measures}

90. In view of the sizeable adjustment needed and the relatively limited scope for further cut in primary public spending, the above expenditure measures will not be sufficient to raise the primary surplus to a level that is commensurate with the objective of reducing the ratio of debt-to-GDP over the medium term. Hence, serious revenue-enhancing measures would need to accompany or even precede some expenditure cuts, given the difficulty in rationalizing public expenditures in the short term.

91. Much has been done in the past few years to improve the tax structure and collection, as shown in the sharp increase in the tax revenue. A number of other structural measures aimed at modernizing the revenue administration towards a function based structure with strong headquarters, fully automated business processes, risk-based compliance programs, and skilled and professional staff, include (i) integrating within the newly created large taxpayer office (LTO) the collection function and introducing further automation including electronic filing (2007); the coverage of the LTO is further expected to be extended to include the VAT, built property tax and some indirect 
taxes; (ii) staffing fully the newly established tax roll department, modernizing and updating the business activity code, and ensuring accurate recording of taxpayers (2007); (iii) finalizing the registration of private and public sector employees in the withheld tax on salaries and wages database, and extending the reformed operations to the regional offices; and (iv) unifying tax procedures under a unique "Tax Procedure Code" which is presently under final revision and discussion in parliament, an important step for the introduction of the Global Income Tax (GIT) in 2008.

92. Any change in tax policy in the context of the present reform program should be guided by the need to minimize distortions and enhance equity and fairness in the distribution of the tax burden, taking into account the broad objectives of this program. In this spirit, the government plans to implement a number of revenue-enhancing measures that would attempt to shift the tax burden to those that are in the high-income brackets.

93. The tax on interest income will be raised from 5\% to $7 \%$ beginning in 2008 . The fact that interest received constitutes an important share of taxable income of those in high income brackets reinforces the argument for this taxation. This tax increase could generate about $0.5 \%$ of GDP in revenue, assuming a 10\% rate of growth of deposits over the medium term (a realistic estimate judging by recent trends), a $72 \%$ dollarization rate, and relatively stable interest rates over the medium term.

94. The introduction of the value added tax (VAT) in early 2002 was a major success, generating $5.1 \%$ of GDP in revenue in 2005. The Lebanese VAT law exempts basic food staples and social items such as education and health services, medicine, books, and public transport, which make the bulk of low-income household consumption of goods and services (it is estimated that about $60 \%$ of their consumption is exempt from the VAT). In this regard, the VAT could be seen as a progressive tax on income spent due to the fact that the goods and services exempted from the VAT represent a relatively smaller portion of spending by high-income households.

95. In this light, the government plans to raise the VAT rate from $10 \%$ to $12 \%$ in 2008 and from $12 \%$ to $15 \%$ in 2010 . Over the medium term, a $15 \%$ VAT will generate additional revenue of at least $2 \%$ of GDP. All this will bring the total contribution of the VAT to the budget to about $7.5 \%$ of GDP by 2010 . For improved collection, the government further seeks to adopt monthly VAT filing for large corporations as of 2007.

96. The contribution of the gasoline excise tax to the budget declined from LL 800 billion in 2003 to about LL 360 billion in 2005 and has almost become nil for the period May-August of 2006 (total collection of car gasoline excises for 2006 is not projected to exceed LL 250 billion) due to the capping of the retail price at the May 2004 level, which has created additional economic distortions and eroded the gasoline excise revenue base. This decision was introduced temporarily on the assumption that oil prices would go back down in the near future. The excise tax will be adjusted gradually beginning in 2007 until it reaches its pre-cap rate by 2011 . At the same time, the cap will be lified to allow a full pass-through of international prices to domestic retail prices.

97. As part of the overall tax reform, the government will replace the scheduler income tax by a global income tax (GIT) by 2008 without any changes in the tax rates. This will aggregate the taxpayer's sources of income, introducing more equality in tax burden. This measure will not impose any new burden on low-income people but will introduce a fairer distribution of the tax burden. Together with the improvement in the overall tax administration and compliance, the reduction in administrative complexities, and the unification of exemptions, the GIT is expected to improve tax revenue. It is estimated that the introduction of the GIT will generate about $1 \%$ of GDP of additional revenue over three years, 2008-10. The government intends to strengthen the administration of the 
property tax and broaden its base. The yield of the property tax in Lebanon, at $0.3 \%$ of GDP, is low compared to developed countries (1.45\% of GDP for OECD).

98. The property tax administration has started the process of updating and building a comprehensive database on built properties to be achieved through a synchronization of information systems between property tax database and Cadastre as a first step, and municipalities at a second stage.

99. In this context, there is a pressing need to settle the long-standing issue of seashore violations through the adoption of the appropriate draft law, which was recently approved by the cabinet. It is expected that at least LL 135 billion will be collected in the form of penalties in 2007. In addition, the government will begin to collect rent on these "leased" properties beginning in 2007 estimated at LL 45 billion a year. In deciding on how much rent to charge, the government will take into account the fact that these properties are not subject to property tax and hence they should yield high rent generating additional revenues for the budget.

100. The government seeks to strengthen its collection of revenues from the various public properties particularly form Casino du Liban through the agreed upon arrangement with its management. This arrangement stipulated that the government that will start collecting $40 \%$ of total net revenues from gambling for the next 10 years instead of $30 \%$ which was collected during the last 10 years. This ratio will be further raised to $50 \%$ before the agreement is terminated and the Casino operation is fully returned to government.

\section{E. Privatization Program}

101. The achievement of government's key objective of a sustainable growth with equity is critically dependent on reducing the level of public debt, so as to bring about lower interest rates to encourage private investment, and to accommodate adequate levels of social expenditures and public investments as a result of an easing of the debt service burden.

102. The privatization component of the government's reform program is key to both the objective of promoting growth and that of reducing debt and the fiscal deficit. It would also contribute to the deepening and expansion of Lebanon's capital markets. The privatization program is expected to improve the reliability and quality of the provision of public services, reduce operating costs through increased efficiency of operations, expand the range of services offered to customers, reduce the cost of services to business, introduce competition and hence improve the competitiveness of the economy.

103. The legal framework for privatization had already been established in late 2000 with the adoption of a framework privatization law (Law 228) that formed the basis for developing sectorspecific laws to underpin privatization operations in various sectors. The Higher Council for Privatization (HCP) was established in the Office of the Prime Minister in 2001 and two sectoral laws were enacted in 2002 to form and privatize a fixed line telecommunications company and to corporatize, unbundle and privatize the power sector. Apart from transport, these two sectors represent by far the largest components of public infrastructure in terms of assets. Both laws provide for the establishment of regulatory bodies for the respective sectors. As mentioned above, privatization did not go ahead during 2003-05 because of political constraints.

104. Preparation for privatization and liberalization in telecommunications has been resumed after the war. The key components of the telecom privatization plan are: (i) the establishment of a regulatory authority and appointment of its board by the first quarter of 2007; (ii) the enactment of a law authorizing the sale of the mobile sector's assets and relevant operating licenses by the first quarter of 2007; (iii) the sale of a majority stake in, or 100 percent of the mobile sector companies by 
the second quarter of 2007; and (iv) the formation of Liban Telecom (as envisaged in Law 431 of 2002), through the incorporation of the fixed line operator Ogero (a Government-owned entity) and two departments of the Ministry of Telecommunications, by mid-2007 and its privatization in 2008 . The HCP is finalizing contracts with reputable international firms to assist with the privatization of the mobile sector and has retained another one to help corporatize Liban Telecom.

105. The privatization of the mobile sector companies will be undertaken in a transparent manner through a public offering that ensures the widest possible stockhoider base. Other than reducing the total debt stock, this operation will play a major role in developing the Beirut Stock Exchange, fueling growth and job creation, and providing mobile telephony services more efficiently.

106. The scope for immediate privatization of the power sector is more limited given the state that EdL is currently in. However, urgent action is needed to reverse the grave fiscal impact of EdL on the budget. Energy generation is far below installed capacity and system losses (technical and nontechnical losses and non-collection of bills) are high. EdL's losses are large even though tariffs are not low by regional standards, and rising fuel prices have compounded the problem. Government is covering EdL's operating losses as well as capital expenditures and debt service, representing outlays of a total of $3.5 \%$ of GDP in 2006 . In the meantime, sector investment requirements for the five years are estimated to be in the order of US\$1.5 billion. Given the heavy debt burden facing the economy and the increased demand for power consumption, it is imperative for the private and not the public sector to provide the needed investments and services in the future.

107. As in the case of telecommunications, legislation adopted in 2002 (Law 462) provides the legal framework for privatization of the sector. The law envisages the creation of a regulatory authority, and the separation of generation, transmission and distribution activities, in anticipation of the creation of one or more public corporations responsible for generation and distribution. Transmission would remain a public function.

108. Privatization of the sector remains the government's objective for the medium term, but in the short run, the focus will be on strengthening sector capacity and reducing losses, with involvement of the private sector where feasible.

109. With respect to other infrastructure, government will prepare legislation in the course of 2007 to support privatization in the water and wastewater sectors, through concessions. In the case of ports and the Beirut Airport, government intends to proceed with privatization through concessions as well.

110. As for sectors other than public infrastructure, the national airline, Middle East Airlines (MEA), was restructured in 2001. The restructuring, which involved major layoffs, has contributed to MEA achieving an operating profit in 2005, despite the rise in fuel prices. For 2006, losses are envisaged as a result of the war. Nevertheless, when market conditions are deemed to be adequate, BdL plans to divest its shares in MEA, as well as its shares in the INTRA lnvestment Company (financial institution), which is a major shareholder in the Casino de Liban. Finally, the Governmentowned refineries, which have not been operative since the 1975-90 war, would be offered for sale to private investors interested in establishing refinery and/or oil storage capacity or in any form of cooperation with the private sector.

111. In terms of early impact on public debt (in 2007 and 2008), only the planned privatization in the telecommunications sector should represent fairly significant amounts. By law, proceeds from all privatization efforts are to be applied to public debt reduction. 


\section{F. Monetary and Exchange Rate Policies}

112. Maintaining macroeconomic stability through a proper monetary and exchange rate policy is a prerequisite for stimulating private sector investment and growth and for maintaining social stability. In the past few years, the central bank (BdL) has played a much greater role than a typical central bank would under normal circumstances. But circumstances were not normal by any means. The difficult political and security situation which manifested itself mainly in higher dollarization and in pressures in the foreign exchange market required the central bank to resort to various (and sometimes untypical) financial and long-term monetary instruments to maintain such stability and avoid a financial crisis. This was very much in evidence following the assassination of Prime Minister Hariri and during the July/August war. The BdL issued dollar denominated CDs to banks and lengthened the maturity of deposits, to help stabilize a difficult situation.

113. With the onset of economic reforms and the expected support of the international community, the central bank will focus its attention on its main mandate of maintaining price stability using only short-term monetary instruments. At the same time, BdL's policy independence will be strengthened mainly through improving the fiscal situation and not resorting to any direct borrowing from the central bank. In an environment of renewed confidence and good coordination with fiscal policy, there is a scope for reducing interest rate spreads. This could be accomplished without necessarily creating much pressure on reserves given the stability of the deposit base, which has proved to be less sensitive to changes in foreign interest rates. In the absence of fiscal tightening and reform, however, the ability of the central bank to influence interest rates is limited given that capital is fully mobile and the exchange rate is fixed.

114. A potential reduction in interest rates-starting with a reduction in the margin on dollar deposits - should boost the demand for credit by the private sector and channel excess liquidity toward productive uses and help the development of capital markets (see above). As the level of international reserves is approaching an adequate level, the need for the central bank to absorb liquidity will diminish and so will the cost of sterilization.

115. As part of its efforts to focus on its main mandate and to rid its balance sheet from assets that do no not typically belong to a central bank (but are there because of special past circumstances), the BdL plans to sell part of its shares in the Middle East Airlines (MEA), and Intra Investment Corporation which includes Casino du Liban by mid-2007, market conditions permitting. In tandem, BdL will put forward a liquidity management strategy to ensure the smooth running of markets given any debt swaps and reductions that may take subsequent to the International Conference in January.

116. The policy of stable exchange rate has served Lebanon well and is critical to maintaining price stability and confidence. While competitiveness is not a concern at this time, structural reforms of the kind included in the economic reform program will enhance competitiveness. Furthermore, to broaden the range of its policy instruments, the central bank will develop the necessary tools to deal with a more flexible exchange rate regime in the future, should the need arise and provided that price stability is not jeopardized.

\section{G. Banking Sector's Contribution to the Domestic Effort}

117. Commercial banks in Lebanon remain profitable and well capitalized by international standards. Asset quality remains good, with less than $10 \%$ ratio of problem loans to total loans. The risk associated with the large exposure to the government is mitigated by the abundant liquidity in the banking system compared to international standards. But this risk is expected to fall due to the ongoing reform efforts and the expected international financial assistance that would reduce the need for government borrowing. The BdL will gradually push banks to become intermediaries in government papers (instead of being the principal holders of these papers), thus reducing their 
exposure to sovereign risk and allowing them to finance private sector activities which would enhance growth and job creation.

118. As for the financial contribution of commercial banks to the adjustment efforts, it will be up to the banking sector to determine the size and modalities of their contribution as was the case at the time of the Paris II meeting. Any voluntary contribution from banks will complement and facilitate the government's reform efforts.

\section{MEDIUM-TERM MACROECONOMIC FRAMEWORK}

\section{A. Macroeconomic scenario with domestic adjustment}

119. The implementation of the government's reform program as outlined above, with early recovery and reconstruction and without further external support, assumes that growth would resume fairly quickly (from the low 2006 base) as of 2007 . Real GDP growth is conservatively estimated to stay in the $4-5 \%$ range over the medium term. These are modest targets given the potential for higher growth rates. However, erring on the side of caution is intended to come up with a set of measures that would safeguard fiscal sustainability in the face of adverse external or internal shocks.

120. The financial impact of the July-August war in terms of additional financing needs for the period 2006-10 is estimated at US\$4.5 billion. This amount represents additional financing requirements over and above what was expected in the pre-war medium term scenario, in the absence of any external support. This represents the cumulative additional fiscal deficits during 2006-10, less grants and financial contribution from the international community for recovery and reconstruction.

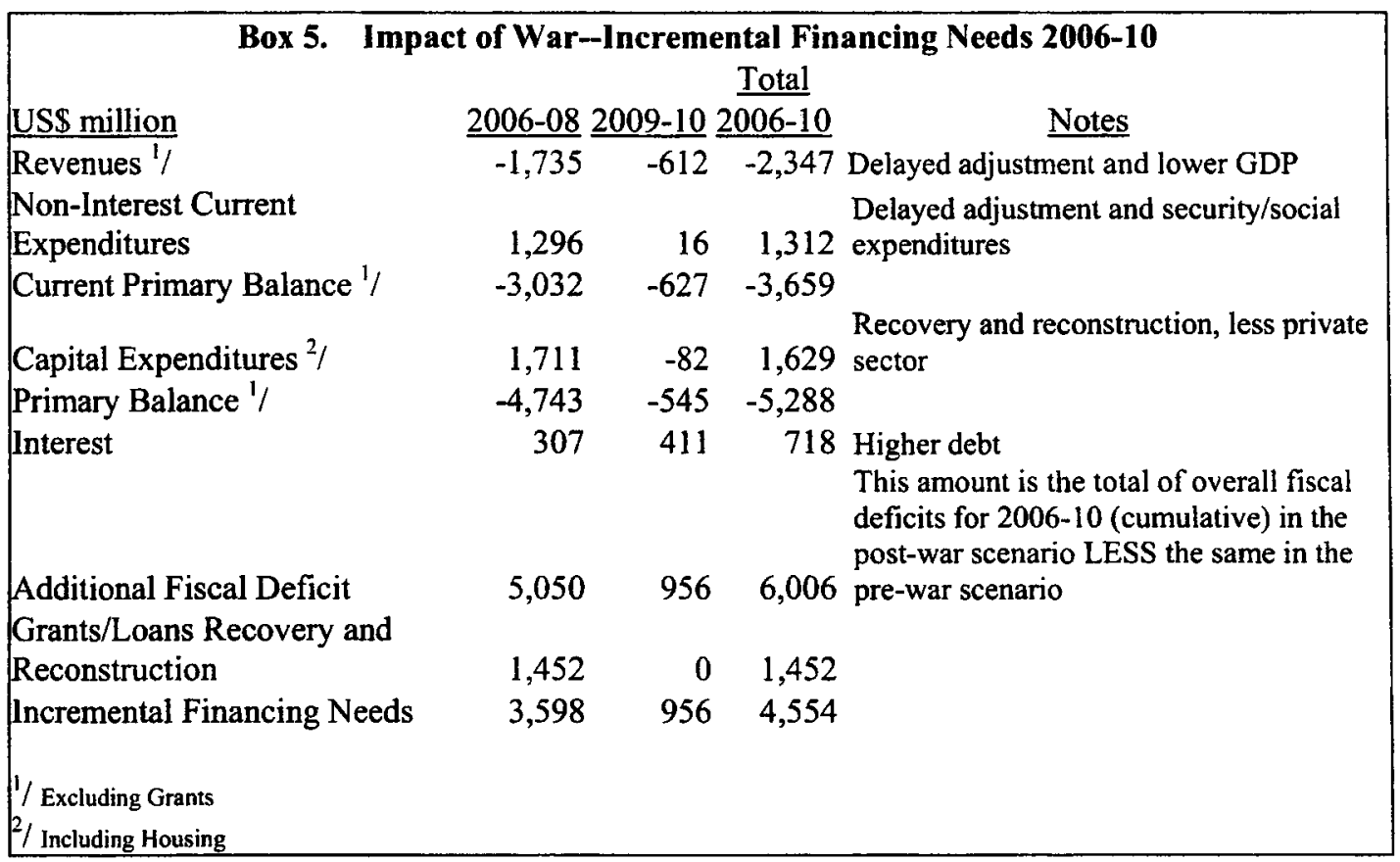

121. Domestic adjustment efforts, in the absence of external support beyond the initial support that has already been pledged for recovery and reconstruction, will lead to significant improvements in macroeconomic balances over the next five years. Under reasonable assumptions on growth, inflation, and interest rates and all things being equal, the primary budget position is expected to increase from 
a $-0.7 \%$ of GDP deficit in 2006 to about $8.9 \%$ of GDP surplus in 2011 , a total adjustment of about 10 $\%$ of GDP. The overall deficit would decline to $2.1 \%$ of GDP with interest payments absorbing slightly less than half of revenues (11\% of GDP). As a result of these macroeconomic and structural adjustments, the debt-to-GDP ratio would decline from about $180 \%$ in 2006 to about $145 \%$ in 2011.

\begin{tabular}{|c|c|c|c|c|c|c|}
\hline Macroeconomic Scenario with dome & tic adju & stment & w/o for & eign ass & istance & \\
\hline & 2006 & 2007 & 2008 & 2009 & 2010 & 2011 \\
\hline Share of GDP (\%) & & & & & & \\
\hline Revenues & 20.8 & 22.1 & 23.4 & 22.9 & 24.4 & 24.5 \\
\hline Grants (recovery and reconstruction) $1 /$ & 2.4 & 3.7 & 0.1 & 0.0 & 0.0 & 0.0 \\
\hline Total Expenditure & 36.9 & 39.3 & 32.7 & 28.7 & 27.8 & 26.7 \\
\hline Current expenditure & 32.3 & 32.3 & 28.6 & 26.1 & 25.1 & 23.9 \\
\hline Non-interest & 19.2 & 19.2 & 16.5 & 14.2 & 13.5 & 12.9 \\
\hline Interest & 13.1 & 13.2 & 12.1 & 11.9 & 11.6 & 11.0 \\
\hline Capital expenditure & 4.6 & 6.9 & 4.1 & 2.6 & 2.7 & 2.7 \\
\hline o/w Reconstruction & 2.2 & 3.7 & 1.0 & 0.0 & 0.0 & 0.0 \\
\hline Primary Balance before grants & -3.0 & -4.1 & 2.8 & 6.0 & 8.2 & 8.9 \\
\hline Primary Balance after grants & -0.7 & -0.3 & 2.8 & 6.0 & 8.2 & 8.9 \\
\hline Interest & 13.1 & 13.2 & 12.1 & 11.9 & 11.6 & 11.0 \\
\hline $\begin{array}{l}\text { Overall Deficit (before recovery/reconstruction } \\
\text { grants) }\end{array}$ & -16.1 & -17.2 & -9.3 & -5.9 & -3.3 & -2.1 \\
\hline Overall Deficit (after recovery/reconstruction grants) & -13.7 & -13.5 & -9.2 & -5.9 & -3.3 & -2.1 \\
\hline Gross Debt (US\$ billion) & 40.3 & 40.5 & 41.3 & 43.0 & 43.9 & 44.7 \\
\hline Gross Public Debt to GDP ratio (\%) & 180 & 170 & 161 & 157 & 151 & 145 \\
\hline Real GDP growth & -5.0 & 4.0 & 5.0 & 4.5 & 4.0 & 4.0 \\
\hline Infiation rate & 7.5 & 2.2 & 2.7 & 2.0 & 2.5 & 2.0 \\
\hline GDP (US\$ billion) & 22.4 & 23.8 & 25.7 & 27.4 & 29.2 & 31.0 \\
\hline $1 /$ Excludes grants provided directly to beneficiaries, private sector and 1 & and Tech & inical As & & & & \\
\hline
\end{tabular}

\section{B. Macroeconomic Scenario with External Support}

122. Reducing the debt-to-GDP ratio to $145 \%$ is a major accomplishment but a debt ratio of such magnitude remains too high by any standard and cannot be sustained over the long term, leaving Lebanon vulnerable to systemic failure. While there is no universal agreement on what constitutes a sustainable debt position, from a practical point of view, it is the level at which the government can satisfy its budget constraint without having to resort to drastic measures or experience difficulties in servicing debt in the future, given reasonable expectations about future fiscal effort.

123. From the operational point of view, public debt in Lebanon is deemed sustainable if the debtto-GDP ratio declines noticeably over time. Assuming an average real growth of $4 \%$, a real interest rate of $6 \%$ and a reduction in the debt to-GDP ratio by about $15 \%$ on account of privatization proceeds, Lebanon has then to maintain a primary surplus of $3.5-4.0 \%$ of GDP to keep the debt-toGDP ratio constant over time. But given the high level of debt and the susceptibility of this debt to exogenous shocks, the objective is to reduce the debt ratio over time by achieving higher primary 
surplus and/or by external financial support. While the adjustment in the primary surplus planned for $2006-2011$ is substantial ( $10 \%$ of GDP), over the next 15 years Lebanon cannot realistically sustain an average primary surplus of more than $3.5-4 \%$ of GDP (or about $2-2.5 \%$ over the period $2010-20$ ) since compressing expenditures and raising additional revenues would become extremely difficult to maintain in view of the country's needs, allowing only a slight decline in the debt ratio.

124. In view of the high level of debt and the ensuing vulnerability with its social and political repercussions, international support would be crucial for Lebanon to secure a continuous and perceptible reduction in the debt-to-GDP ratio over the long run. Significant external support in the form of grants and highly concessional loans would substitute part of the new market borrowing with a significant impact on debt dynamics especially if this support is frontloaded. While grants would have the greatest impact on the debt stock, highly concessional loans would affect the debt ratio through a reduction in debt service. Al the Paris II meeting, the international community extended long-term concessional loans at an interest rate that was about half the rate at which Lebanon could borrow at that time. To receive the same degree of concessionality granted under Paris II, under the present circumstance, Lebanon hopes to receive large grants in substantial amounts and in addition to concessional loans at an interest rate in the range of $3-4 \%$ (close to donors' cost of fund) and a maturity period of at least 15 years with a grace period of 5 years.

125. Without international support, the debt-to-GDP ratio will likely revert back to an unsustainable path especially in the face of any exogenous shock unless further fiscal adjustments are taken. However, further cuts in spending or more increases in taxes would not be possible without stifling economic activity or provoking social and political unrest, which would negate fully the benefits of reform.

126. More importantly, international financial support will help create the right environment for reform and contribute significantly to increasing growth and reducing interest rates, which in turn will have a greater impact on debt. For every one percent increase in the growth rate, the debt-to-GDP ratio would decline by about $1.5 \%$ a year. Similarly, a reduction in the real interest rate by one percentage point would have a similar impact on the debt ratio. A combination of both would likely reduce the debt ratio considerably to sustainable levels over the next $10-15$ years.

127. The government plans to place all financial contributions in a special account at the central bank earmarked exclusively for debt service and principal repayments. Any future primary fiscal surpluses would also accrue to this account. This account will also be used for privatization proceeds for the exclusive purpose of debt reduction.

\section{C: Implementation of the reform program}

128. Given the breadth of the reform program and the need for timely implementation over the next five years, a committee representing the ministry of finance, the ministry of economy, and the central bank will be established to ensure proper monitoring of various measures of the program. This committee will prepare a detailed timetable for implementation and work closely with other ministries such as social affairs, health, education and labor to ensure that all actions on the social front are being implemented on a timely basis. The committee will report directly to the prime minister, who will follow up with ministries and agencies not abiding by the agreed timeline under the program while keeping the Council of Ministers fully informed of any developments. 
129. The commitlee will publish a semi-annual report on the status of implementation of the program. In case of delays, the report will explain the reasons and set a new timeframe for implementation. If there are fundamental reasons for a shift in the strategy, then the government will consult with parliament and Lebanon's partners, including international financial institutions and key donors, before a new strategy is adopted.

\section{Conclusion}

130. In this paper, the government of Lebanon presents a comprehensive economic reform program that aims at boosting economic growth, creating employment, maintaining social and political stability, and moving the country to a prosperous era following 30 years of civil strife, Israeli occupation, and unsettled political situation. One first step in tackling the formidable challenges that lie ahead is to address upfront the main source of vulnerability represented by large fiscal deficits and high levels of debt and debt service. If these risks are not addressed, they would lead to unsustainable debt situation and give rise to social and political tensions.

131. This reform program attempts to address the repercussions of thirty years of destruction and suffering and comes in the aftermath of a major devastation caused by the July-August Israeli war. This war increased the burden of public debt and required even more drastic fiscal adjustments than envisaged before the war. Without significant external support, Lebanon would again enter into a vicious cycle of rising debt, high interest rates, depressed private investment and growth that remains well below its potential. Such trend, if not reversed, could easily lead to economic, social and political instability.

132. Assuming no adverse developments occur and provided the government delivers on all its reform measures, the domestic effort will lead to significant reductions in the budget deficits and improvement in the primary surplus amounting to about $10 \%$ of GDP; nevertheless, these efforts alone will not be sufficient to reverse the debt dynamics. All things being equal, the debt-to-GDP ratio will remain at best at about $145 \%$ of GDP and the debt service will still eat up about $50 \%$ of total revenue--a very high level of debt by international standards that could not be sustained in the long term. Generating larger primary surpluses over the long term would not be possible without upsetting the delicate social and political balance in the country.

133. Hence to reduce the debt-to-GDP ratio over time, support the efforts of the Lebanese government, and improve the chances of success of the economic reform program, Lebanon will need the support of the international community. Lebanon hopes to receive financial assistance mainly in grants and highly concessional loans to bring down the debt-to-GDP ratio to a sustainable level, placing Lebanon on a promising and sustainable path out of the debt overhang.

134. Lebanon once again is at a crossroad. It is facing the task of recovery and reconstruction, but this time against a background of public debt that stands at close to twice the level of GDP, and in the aftermath of a massive war that has derailed a promising path. The government is committed to implement it pre-war reform program, although with some re-phasing to take account of the impact of the war. But what the government can do will fall far short of what is needed, and it therefore looks to the international community to provide generous financial support and invest in Lebanon's future. 


\section{ATtaChment IV. Lebanon: TeChNiCAL MEMORANdum OF UNDERSTANding}

\section{INTRODUCTION}

1. This memorandum sets out the commitments of the Lebanese authorities for the period March 31-December 31, 2007 relating to the Emergency Post-Conflict Assistance (EPCA) requested in the Letter of Intent (LOI), and described in the authorities' program. Section II defines the indicative quantitative targets and relative adjustors, reported in Attachment I, and Section III establishes the content and frequency of the data to be provided to IMF staff for monitoring the program.

\section{QUanTitative INDICATIVE TARgets}

\section{A. Definitions and Concepts}

2. Test dates. Quantitative indicative targets are set quarterly through end-2007, and are to be met at the end of each quarter unless otherwise specified.

3. Government. For the purposes of the program, "government" includes the central government, the Council for Development and Reconstruction (CDR), and the grant-financed operations of the Higher Relief Commission (HRC). It excludes all other agencies not specifically listed, including, but not limited to, the following: Electricité du Liban (EDL), municipalities, the Régie, and NSSF. The government balance is defined on a modified cash basis to include foreign-financed expenditure of the CDR, all operations of the HRC, any treasury advance, and arrears (as defined below). Unless otherwise specified, fiscal data are compiled according to the Government Finance Statistics (GFS) manual 1986.

4. Project grants are defined as those grants to the government linked to specific project financing, and do not include grants received for general budgetary support. Budgetary grants are grants received for general budgetary support, including grants in support of specific budget lines and grants to be applied to debt reduction.

5. Arrears. Domestic arrears are defined as government expenditures that have not been paid within 90 days of a payment order being issued. External arrears are defined as overdue payments (principal or interest) on external debt contracted or guaranteed by the government or the Banque du Liban (BdL).

6. Valuation changes. All data are expressed in Lebanese pounds (LL), unless otherwise indicated. To exclude foreign exchange valuation effects: (i) gold will be valued at the December 31, 2006 price of US\$ 629.70 per fine troy ounce; (ii) the U.S. dollar value of foreign assets and liabilities will be converted into $L L$ at the exchange rate of US\$1 = LL 1507.5; and (iii) all foreign assets and liabilities denominated in currencies other than the U.S. dollar will be converted into U.S. dollars and/or LL at their respective exchange rates prevailing as of December 31,2006, as published in the IFS. 


\section{B. Indicative Targets}

7. Target 1. For the purpose of the program, the Gross Reserves (GR) of the BdL (floor, U.S. dollars) are defined as BdL's callable foreign exchange deposits abroad, foreign exchange holdings (including SDR), gold, Eurobonds issued by the Republic of Lebanon (henceforth "Eurobonds"), and holdings of investment grade liquid foreign currency-denominated securities. Encumbered assets will be excluded from GR. The floor for GR will be adjusted (i) downward (upward) for the full amount of any shortfall (excess) in disbursements of official grants and loans to the public sector (government and non-financial public sector) relative to those projected in the program; and (ii) downward (upward) for the full amount of sale (purchase) by the BdL of Eurobonds relative to the December 31, 2006 level of BdL holdings.

8. Target 2. The total net debt of the government (ceiling) is defined as the sum of the domestic currency value of (i) net BdL claims on central government and EdL; (ii) net claims on the government by the rest of the banking system; (iii) net claims on the government by public sector institutions; (iv) net claims on the government by other creditors, including leasing; minus (v) the balances in the CDR and HRC transit accounts at BdL. For the purpose of the program, any transfers from the BdL other than those related to gold revaluation and realized profits will be treated as net government borrowing from the $\mathrm{BdL}$ and added to net BdL claims on central government. The ceiling for net debt will be adjusted (i) upward (downward) for lower (higher) disbursements of budgetary grants relative to the amounts projected under the program; (ii) upward (downward) for lower (higher) receipts from privatization or securitization operations relative to the amounts projected under the program; and (iii) upward (downward) for lower (higher) transfers from BdL's gold revaluation relative to the amounts projected under the program.

9. Target 3. The primary balance of the government, before grants (floor) is defined as the overall fiscal balance on a modified cash basis minus interest payments and grants received. Securitization proceeds will not be counted as above-the-line revenue. For the purpose of the program, transfers from BdL's gold revaluation and transfers not related to realized profits will be considered to be capital (below the line) transfers and therefore excluded from the primary balance.

10. Monitoring of the primary balance before grants, for the purpose of the program, will be carried out from the financing side. The overall balance on a modified cash basis is calculated as the sum of the following: (i) the change in net BdL claims on the central government and EdL; (ii) the change in net claims on the central government by the rest of the banking system; (iii) the change in net claims on the central government by public sector institutions; (iv) the change in net claims on the central government by other creditors; (v) the net change in government arrears; (vi) proceeds from exceptional financing (items such as Paris III grants, privatization revenues, gold or foreign exchange revaluation proceeds, and securitization proceeds); minus (vii) the net change in the CDR and $\mathrm{HRC}$ accounts at the BdL. Budgetary and project grants to the government will also be measured through BdL. All changes will be calculated as the difference between end-period stocks, net of any valuation changes resulting from currency movements and changes in 
accrued interest reflected in the gross debt stock that are reported separately by instrument. For the purpose of the program, interest payments will be measured on a cash basis.

11. The floor on the primary balance before grants will be adjusted (i) downward (upward) for higher (lower) than projected project grant disbursements; (ii) downward for the net amount of the revenues lost due to privatization; and (iii) downward for the full amount of the revenues to be securitized through the associated Special Purpose Vehicle (or other legal instrument).

12. Target 4. New gross domestic arrears of the government (continuous ceiling) are defined as the change in gross domestic arrears arising after February 28, 2007. "Gross" arrears indicates that the taxes and contributions owed to the government are not netted out of the arrears.

13. Target 5. New external arrears of the government and the BdL (continuous ceiling) are defined as the change in external arrears after December 31, 2006.

14. Target 6. Net government borrowing from the BdL (ceiling) is defined as the difference between the BdL claims on the government, in domestic and foreign currency, including all holdings of government securities; and the deposits in domestic and foreign currency of the government at the BdL. For the purpose of the program, any transfers from the $\mathrm{BdL}$ other than those related to gold revaluation and realized profits (e.g., from securitization of future profits) will be treated as net government borrowing from the BdL. The ceiling for net government borrowing from the BdL will be adjusted: (i) downward (upward) for the full amount of any excess (shortfall) in transfers from the BdL's gold revaluation relative to those projected under the program; and (ii) downward (upward) for higher (lower) than projected budgetary grant disbursements.

\section{REPORTING REQUIREMENTS}

15. To permit the monitoring of developments under the program, and as part of and in addition to the regularly reported data, the government will provide to Division $B$ of the Middle East and Central Asia Department of the IMF the information specified below and summarized in the reporting templates:

- Gross Reserves and components (monthly).

- Monetary aggregates, including currency in circulation, M3 and non-resident deposits (weekly).

- BdL balance sheet, including a break down of all assets and liabilities by instrument, by currency and remaining time to maturity (monthly).

- Data on outstanding BdL instruments by type (coupon or discount), currency, amounts, remaining maturity, and yield (monthly). Data on auctions or sale of CDs by the BdL, including amount, maturity, yield, and any premium paid (monthly). 
- $\quad$ BdL income statement on an accrual basis (quarterly).

- Commercial banks' balance sheet (monthly).

- On a checks-issued basis, summary budget operations, revenues, expenditures (including net advances), net domestic financing, balances in the government accounts with the BdL and commercial banks (including privatization accounts), the receipt and use of privatization proceeds (monthly), monthly cash data on foreignfinanced capital expenditures, and relief and reconstruction expenditures.

- Government debt and financing: Government debt statistics as per the usual reporting format provided by the BdL, including BdL lending to EdL. Government interest expenditure on a cash basis by debt instrument and currency. Grants received, of which project-related grants. Privatization proceeds. Valuation changes to the outstanding stock of debt due to currency fluctuations. Changes in the outstanding stock of accrued interest that is reflected in the debt stock. The new stock of domestic and external arrears. Any put or call options, collateral guarantees, warrants or similar derivative arrangements entered into by the government or the BdL. On-lending operations of the government. Securitization of future payment flows to the government or resulting in future payment flows to or from the government. Letters of credit entered into by EdL (monthly). Amortization payments by debt instrument.

- The annual accounts of the NSSF, including the disaggregated accounts of each of the three NSSF funds and any contractual arrears (as defined above) from the central government on NSSF contributions.

- Foreign assistance received (separating grants from loans and budget support from project financing), and full terms of the newly-contracted but not disbursed loans (monthly).

- Balance of payments (current, capital, and financial accounts) (monthly);

- List of short, medium, and long-term public or publicly guaranteed external loans contracted during each quarter; identifying, for each loan: the creditor, the borrower, the amount and currency, the maturity and grace period, and interest rate arrangements (quarterly).

16. Weekly data and data on the central bank CD auctions should be sent to the Division B of the Middle East and Central Asia Department (MCDDB) with a lag of no more than one week. Monthly and quarterly data should be sent within a period of no more than four weeks, except for balance of payments data, which should be sent within a period of no more than eleven weeks. Any revisions to previously reported data should be communicated to the MCDDB in the context of the regular updates.

17. The authorities will prepare and send to MCDDB reports, with appropriate documentation, indicating progress achieved, explaining any deviations relative to the initial 
targets, and specifying expected revised completion dates of the monitorable actions specified in Section III.

18. Details on major economic and social measures taken by the government that are expected to have an impact on program sequencing (such as new legislation on decrees) will be sent in a timely manner to MCDDB. 


\section{IMF Executive Board Approves US\$76.8 Million In Emergency Post-Conflict Assistance to Lebanon}

The Executive Board of the International Monetary Fund (IMF) approved today an amount equivalent to SDR 50.75 million (about US\$76.8 million) in Emergency Post-Conflict Assistance (EPCA) to Lebanon in support of the authorities' economic program for 2007.

The objectives of the IMF supported program are to protect financial stability, contain the budget deficit, and to initiate structural reforms that are crucial to the success of the authorities' medium-term reform program for 2007-2011. The EPCA would provide an appropriate support for the transitional challenges of moving from post-conflict situation to a program of fiscal adjustment starting in 2008. The IMF's support through EPCA is a key part of a concerted international effort to provide financial assistance to Lebanon.

Following the Executive Board's April 9 discussion on Lebanon, Mr. Murilo Portugal, Deputy Managing Director and Acting Chair, stated:

"The July 2006 conflict imposed a heavy human and economic toll on Lebanon, with severe damage to infrastructure, the displacement of people, and a slow down of economic activity. At the same time, the country's financial markets weathered the crisis well. Financial stability and confidence in the exchange rate peg were maintained owing to the central bank's skillful management, the banking system's strong liquidity position, and the timely deposits by Saudi Arabia and Kuwait in the central bank.

"2007 will be a difficult transition year. Prospects for an economic recovery in 2007 are weak owing to the ongoing political tensions. The authorities face a difficult post-conflict environment, as well as challenges stemming from a large debt overhang and financial vulnerabilities. Against this background, the authorities have developed an ambitious medium-term reform program that was supported by donors at the Paris III conference. The program aims at significantly reducing the debt-to-GDP ratio over the next five years. Support from donors in the form of grants and concessional lending will assist in achieving these objectives. Part of the amounts pledged at the Paris III conference will be available for debt reduction, and the rest for project financing. 
“The authorities' program for 2007, which will be supported by the Fund's Emergency PostConflict Assistance, focuses on maintaining financial stability and containing the primary fiscal deficit while accommodating reconstruction and relief spending. Keeping the excise rates on gasoline products at the levels prevailing in March will be a key measure to achieve the deficit objective. The authorities' financing strategy for 2007 relies on the timely disbursement of donor support, which is also important in limiting the further build-up of government debt.

"In the course of 2007, the authorities plan to initiate important structural reforms that will support their medium-term reform and debt reduction objectives. These include actions to strengthen public financial management so as to better align spending with budget priorities; reforms in the social and energy sectors with a view to containing open-ended government transfers to the social security system and to the power utility; and introduction of a global income tax, which would lead to a more equitable sharing of the burden of fiscal adjustment.

"The authorities are committed to exchange rate stability. Given the domestic and regional political environment, Lebanon remains highly exposed to possible swings in confidence. Thus, maintaining a comfortable level of international reserves will remain a key program objective. The authorities consider that, if financial pressures re-emerge, the exchange rate peg can be protected by the international reserves buffer and interest rate increases, but they recognize that if pressures persist, a faster pace of fiscal adjustment may be needed," Mr. Portugal said. 

